This document is the Accepted Manuscript version of a Published Work that appeared in final form in Chemical Reviews 114, 5641 (2012), copyright (c) American Chemical Society after peer review and technical editing by the publisher. To access the final edited and published work see

http://pubs.acs.org/doi/abs/10.1021/cr300144z 


\title{
Theoretical Studies of Spectroscopy and Dynamics of Hydrated Electrons.
}

\author{
László Turi ${ }^{\mathrm{a}, 1}$ and Peter J. Rossky ${ }^{\mathrm{b}, 1}$ \\ ${ }^{a}$ Eötvös Loránd University, Department of Physical Chemistry, Budapest 112, P. O. Box 32, \\ H-1518, Hungary \\ ${ }^{\mathrm{b}}$ Department of Chemistry and Biochemistry and Institute for Computational Engineering and \\ Sciences, \\ University of Texas at Austin, Austin, TX 78712
}

\footnotetext{
${ }^{1}$ E-mail: turi@chem.elte.hu, fax: (36)-1-372-2592

rossky@mail.utexas.edu, fax: (1)-512-471-6835
} 


\section{Table of contents}

1. Introduction 4

2. Experimental techniques: a brief historical overview 8

3. Theoretical approaches 12

3.1 Static computational techniques

3.2. One-electron statistical, finite temperature approaches: adiabatic methods 14

3.3. Extensions of one-electron adiabatic approach 20

3.4. Ab initio molecular dynamics techniques 22

3.5. Non-adiabatic (NA) molecular dynamics methods 24

3.6. Quantum simulations of non-adiabatic events with nuclear

quantization: the correlation function approach 27

4. Equilibrium structure and energetics of the hydrated electron 30

4.1. Structural models and energetics of the hydrated electron 30

4.2. Structure and energetics of water cluster anions

5. The equilibrium optical absorption spectrum of the hydrated electron 47

6. On other dynamical aspects of the equilibrium hydrated electron 53

6.1. Solvation dynamics and equilibrium energy fluctuations 53

6.2. Hydrated electron diffusion 56

6.3. Vibrational density of states of the hydrated electron 58

6.4. Hydrated electron simulations at different thermodynamic state points $\quad 59$

7. Non-equilibrium hydrated electron dynamics and spectroscopy in bulk, in clusters and on water/air infinite interfaces $\quad 61$

7.1. Molecular dynamics simulations of excess electron photoinjection in neat water

7.2. Molecular dynamics simulations of photoexcitation experiments of 
an equilibrium hydrated electron

7.3. Non-equilibrium molecular dynamics simulations of excess

electrons in water clusters and on water/air interfaces

$\begin{array}{lll}\text { 8. } & \text { Related topics } & 78\end{array}$

8.1. Hydrated electrons in the dynamics of CTTS states of halide ions 78

8.2. Ab initio molecular dynamics studies of hydrated electron reactivity $\quad 80$

8.3. The dynamics and reactivity of the hydrated dielectron 81

9. Summary and outlook $\quad 86$

10. Acknowledgments $\quad 89$

$\begin{array}{lr}\text { 11. Tables } & 90\end{array}$

12. Figure Captions, Figures $\quad 92$

13. References 114 


\section{Introduction}

The hydrated electron is a fascinating species that has captured the scientific attention of chemists for more than fifty years. Although the experimental detection of the hydrated electron dates back only to $1962,{ }^{1}$ the existence of the hydrated electron has been continuously speculated since the experimental observation ${ }^{2}$ and postulation ${ }^{3}$ of the first solvated electron species, the ammoniated electron (an excess electron solvated in liquid ammonia). Water radiolysis generated an immense early interest in identifying the analogous aqueous species and understanding its physical properties. The postulation, ${ }^{4,5}$ and later the discovery of the hydrated electron ${ }^{1}$ was quickly followed by extensive studies of its chemical reactivity. ${ }^{6,7}$ The hydrated electron is the simplest reducing agent in chemistry. Its important role, mostly as a highly reactive intermediate in physical, chemical, and biological processes, as well, has been recognized. ${ }^{8}$ The localization and the hydration of excess electrons are also of fundamental importance in condensed phase chemistry and physics with direct implications in, among other fields, electrochemistry, photochemistry, radiation chemistry and electron transfer in condensed phases and in biological systems. ${ }^{9}$

Despite this long history, the hydrated electron system has proved to be resistant to efforts to unveil a fully detailed microscopic picture underlying the experimental observations. The difficulty partly stems from the fact that hydrated electrons can be observed in diverse environments of various size and dimensionality: in the bulk, in finite size molecular clusters, at water/air interfaces, and in thin water layers deposited on metals. The scientific challenge of the problem, combined with the re-discovery of the fundamental importance of the hydrated electron system, has initiated the latest wave of intense scientific interest and has resulted in a series of high profile research articles. ${ }^{10,11,12,13,14,15,16,17,18,19}$ Both the most sophisticated experimental techniques and theoretical algorithms have been 
employed in this recent, seemingly evergreen, research area. Although the perspective to find a fully detailed comprehensive explanation for the diverse properties of the hydrated electron is promising, ${ }^{20,21}$ even some of the most elementary questions are still under heated debate. The latest review articles on the hydrated electron express this persistent interest quite entertainingly by characterizing the electron as "Nature's most squishy ion"22 and analyzing "electron promiscuity". 23

Experiments and theory contribute in parallel to the understanding of this species. Since in many cases hydrated electron properties are reflected only by indirect experimental information, theory has a central role in properly interpreting these data. Furthermore, continuing experiments have been strong motivators of the development and subsequent application of new methods and algorithms that are capable of reproducing hydrated electron behavior with increasing precision and reliability. On the other hand, improving theoretical predictions have raised unexpected new challenges and questioned some models underlying the interpretation of experiment, challenges and questions that can only be definitively validated by performing careful and suitably designed new experiments.

The hydrated electron forms a model system in several respects. Besides being the simplest reducing agent, the hydrated electron can be thought of as the simplest possible electronically active solute, having only a single electronic degree of freedom. The coupling of the excess electron state(s) to solvent dynamics is directly manifest in hydrated electron dynamics. This anticipated relative simplicity is an attractive feature of the hydrated electron system, and it makes the hydrated electron an ideal candidate for a symbiotic interplay between experiment and theory. The deceptiveness of this simplicity, however, has added to the intellectual challenge to understand the hydrated electron in satisfactory detail.

The present paper focuses on the theoretical side of the development of hydrated electron theory. Here, we illustrate the key role theory has been playing in understanding the 
molecular level physics of the hydrated electron. In particular, we emphasize the theoretical studies devoted to interpreting and/or predicting the spectroscopy and dynamics of the hydrated electron. Such important and controversial topics as the structure and energetics of the hydrated electron are included, as these properties are deeply connected to and underpin the molecular level dynamics of the system, and, as such, must be discussed in significant detail. The review of the theory, nevertheless, would not be complete without continuous references to available experimental data.

The paper begins with a short historical overview of the experimental methods to investigate hydrated electron systems. In section 3, the main theoretical approaches employed for the hydrated electron system will be summarized. Here the discussion will also include a critical analysis of the applicability of the theoretical procedures. Next, in section 4, we review equilibrium hydrated electron properties and, in particular, their connection to the dynamics of the system. First, the structure and energetics of the hydrated electron will be overviewed in the context of experimental observations. The results for the bulk hydrated electron and hydrated electron clusters (water cluster anions) are discussed in separate subsections, although the intimate connections between these systems will be emphasized. We devote section 5 to the optical absorption spectrum of the equilibrium hydrated electron (in bulk, clusters and interfaces), another controversial and open issue. Here, we explicitly point out the dynamical character of the problem. Other related equilibrium solvent and electronic dynamics aspects are collected next, in section 6, including the calculation of the electron's diffusion coefficient and the equilibrium electronic energy fluctuations. We then turn to nonequilibrium dynamical behavior in section 7. In particular, we collect the simulated results corresponding to time-resolved laser experiments (photoionization, photoinjection and photoexcitation) carried out using adiabatic and non-adiabatic molecular dynamics simulation techniques. Once again the results for bulk hydrated electron and water cluster anions will be 
discussed separately. Next (section 8), the simulation results of the most recent research efforts on hydrated electron chemical reactivity will follow briefly. The issues concerning a strongly related exotic species, the hydrated dielectron will be also overviewed. Finally, in section 9, we summarize the status of the topic and briefly discuss the open issues and challenges that remain to be tackled in the future. 


\section{Experimental techniques: a brief historical overview}

This section collects the main experimental techniques used in hydrated electron investigations. The focus here will be on reviewing the various techniques and the results of relevant experiments; more detailed technical discussions, when necessary, are given later in direct context of the corresponding theoretical results.

Due to its high reactivity, the lifetime of the hydrated electron is in the microsecond regime at room temperature even in pure water. This fact alone explains why it was difficult to detect the dynamics of the hydrated electron in the early days of its history. Nevertheless, hydrated electrons can be trapped as stable species in low-temperature crystals and glasses. ${ }^{24}$ At these low temperatures, the magnetic properties associated with the excess electron spin can be easily detected. In fact, electron-spin resonance (ESR) provides the most direct information on the structure of the hydrated electron. ${ }^{25,26}$ Since the hydrated electron possesses an extra charge, the classical method of conductometry can be utilized to investigate hydrated electron mobility and diffusion in the liquid phase. ${ }^{27}$ Ambient and higher temperature measurements necessarily require more advanced time-resolved experimental methods. Time-resolved optical spectroscopies mainly monitor time-dependent absorption spectra and have become the main experimental information source on the hydrated electron. As a result of the rapid advances in the field, early picosecond pulse radiolysis studies $^{28,29,30,31,32}$ were soon followed by ever faster sub-picosecond time-resolved optical laser spectroscopy investigations ${ }^{33,34,35,36,16}$ providing detailed energetic and dynamical information on the hydrated electron system. The temporal evolution of the spectral features evidences that the fastest dynamical events of electron hydration take place on the femtosecond timescale. Presently, the available time-resolution penetrates to the sub-100 fs 
regime. ${ }^{36}$ The use of $\mathrm{x}$-ray absorption and the detection of core-hole decay open a window on detecting few hundred attosecond events. ${ }^{37}$

There are several routes to monitor electron solvation dynamics in bulk water experimentally. ${ }^{38,39}$ The observed dynamics directly reflect the way the non-equilibrium excess electron was prepared. One group of initial conditions includes precursors to equilibrium hydrated electron formation, such as molecular water excited states and conduction band electrons. The molecular excited states are typically produced by nearthreshold 2-photon excitation of water while the higher energy electrons can be obtained by direct ionization of bulk water or of electron donor solutes in an aqueous environment. The second group involves well-defined optically excited states generated from an existing equilibrated hydrated electron. In all cases, the dynamics of the ejected or excited electron is subsequently monitored, with the spectral evolution to the signature of the completely equilibrated ground state hydrated electron, an intense but broad, asymmetric and featureless optical band with a maximum at $1.72 \mathrm{eV}(720 \mathrm{~nm})$ at room temperature as shown in Figure 1. ${ }^{40,41,42}$ The two scenarios sample different relaxation channels and provide complementary information on excess electron relaxation in water. More recently, femtosecond laser spectroscopy experiments with selective electron scavengers which specifically probe the presence of the excited state hydrated electron directly have been designed and carried out. ${ }^{16}$

A more detailed understanding of electron solvation can be attained, in principle, by extending the investigations from bulk water to hydrated electron clusters and hydrated electrons on or near water/air interfaces. Negatively charged water clusters were first detected in $1981 .^{43}$ Water cluster anions since then have become a favorite target for experimentalists. $44,45,46,47,48,10,11,12,13,49$ A practical advantage of cluster investigations is that due to the more limited number of nuclear degrees of freedom, the experimental spectroscopic signatures are more clearly observed and interpreted in clusters. Furthermore, since the cluster and bulk 
behavior should, in principle, converge in some sense for large clusters, extrapolating cluster properties to infinite size clusters should connect the bulk and the clusters.

In water cluster anion experiments, electron attachment takes place in an expanding molecular jet. The dynamics of the formation of hydrated electron clusters, the finite size analog of hydrated electron formation following electron ejection into bulk water, is a process of considerable interest in itself, but it has not been directly investigated experimentally and is equally difficult to simulate. In hydrated electron cluster experiments, time-of-flight mass spectrometric anion size selection is typically combined with photoelectron spectroscopy. The principal quantity detected via photoelectrons is the mass-resolved vertical electron detachment energy (VDE), the energy needed to remove the excess electron from the cluster without changing its nuclear configuration. The experimental detection of excited state dynamics of water cluster anions following optical excitation has become possible in the last few years using remarkable time-resolved photoelectron imaging in pump-probe laser experiments. $^{11,13}$

In relatively new developments, hydrated electrons were generated and observed experimentally on water/air interfaces and in thin water layers. At very low temperatures, excess electrons have been detected and characterized on crystalline and amorphous ice surfaces that were deposited on metals. ${ }^{50,51}$ Electron solvation in thin water layers were also experimentally studied on $\mathrm{TiO}_{2}$ surfaces using two-photon time resolved photoemission spectroscopy. ${ }^{15,52,53}$ Most recently, liquid water microjets under ambient conditions have been interrogated using ultrafast photoelectron spectroscopy and via second harmonic generation to study interfacial hydrated electrons. ${ }^{18,54,55,56}$

The experimental techniques listed up to this point measure signals that are directly associated with the properties of the excess electron. Nevertheless, it is also informative to probe the water molecules that interact with the hydrated electron. In particular, it is widely 
appreciated that the character of solute-solvent interactions is reflected in molecular vibrational frequency shifts. Relatively recently, time-resolved resonance Raman spectroscopy measurements have been used to probe water vibrations in the bulk hydrated electron system and have provided important insight into the structure and dynamics of the hydrated electron. ${ }^{57}$ 


\section{Theoretical approaches}

We now overview the most important theoretical methods in hydrated electron theory. On the theoretical front, there are two main approaches to the hydrated electron problem, the static and the statistical approaches. In the static route, the hydrated electron potential energy landscape is studied with computational methods of increasing complexity and sophistication, emphasizing electronic structure methods. The earliest and more recent continuum and semicontinuum methods, as well as electronic structure calculations, including high level ab initio approaches and density functional techniques, all belong in this category. In the second group of approaches, finite temperature and the statistical mechanics of the system takes the forefront; statistically averaged properties are computed over an ensemble of hydrated electron systems generated with Monte Carlo or molecular dynamics simulation techniques. Due to the inherent quantum nature of the excess electron, purely classical methods are of no use here, and electronic structure must be introduced, at least approximately. We will further separate the discussion of the statistical methods based on whether they treat only one electron quantum mechanically or implement a many-electron description of the system.

\subsection{Static computational techniques}

The static computational methods focus on systematically improving and exploring the accuracy of the description of the potential energy surface governing hydrated electron geometry. Usually two major obstacles play a role in association with this type of methodology. First, the system size that can be realistically investigated with satisfactory precision is seriously limited when using the most reliable computational methods. Second, since these calculations mostly locate stationary points on the potential energy surface, they necessarily cannot address the statistical aspects (ensemble averages and/or dynamics) of the 
system. From this latter perspective, the general appraisal of the static methods is beyond the scope of the present review. However, since sophisticated $a b$ initio calculations provide important benchmarks for the models used in statistical methods, a limited discussion of relevant electronic structure calculations is necessary. We also point out some connections between seemingly different theoretical approaches.

From a historical point of view, theoretical studies of the ammoniated electron well preceded those of the hydrated electrons. These earliest calculations introduced the so-called cavity model, well before the experimental observation of the hydrated electron. The first cavity model for the solvated electron was essentially a "particle-in-a-box" model with only some unspecified strong short-range interactions. ${ }^{58}$ The improved cavity model of Jortner included the binding of the electron by the polarized dielectric medium, ${ }^{59}$ similar to the polaron model of Landau. ${ }^{60}$ Further development of the solvated electron theory was based on the cavity picture, and soon included models for the hydrated electron, as well. In the hierarchy of increasing complexity, continuum models ${ }^{59,61}$ were followed by semicontinuum models $^{62,63,64}$ that added an atomistic description of the solvent molecules in the first shell. The early theoretical models and their merits and limitations were reviewed by Feng and Kevan. ${ }^{65}$ Dielectric continuum theory, which treats statistical thermal effects implicitly, still plays an important role in elucidating hydrated electron properties, as exemplified by the work of Makov and Nitzan, who studied solvation and ionization near a dielectric surface, ${ }^{66}$ with implications for electron hydration in finite size hydrated electron systems. More recently, solvation dynamics of an excess electron in a polarizable dielectric medium has been studied based on an extended continuum model via its polarization relaxation function. ${ }^{67}$

The classic studies of Newton ${ }^{64}$ marked the beginning of the application of ab initio quantum chemistry calculations on hydrated electron systems. Since the number of water molecules that can be treated explicitly by ab initio methods is necessarily severely limited, 
the rest of the solvent is either neglected (cluster calculations) ${ }^{68,69}$ or taken into account in some approximate ways. ${ }^{64,70}$ Calculations in the earliest studies were performed at fixed molecular geometries that correspond to particular anticipated cavity type molecular arrangements. $^{64,68}$ The investigated systems usually contained 3-8 water molecules and an extra electron. Later geometry optimization allowed localization of minimum energy configurations on the potential energy surface. Electron correlation has also been recognized to be of primary importance in the hydrated electron system, thus Hartree-Fock (HF) calculations ${ }^{64,68,69}$ have been soon followed by the application of higher level methods, mainly at the MP2 level. ${ }^{71,72,73,74,75}$ Static density functional theory (DFT) quantum chemistry computations have also been performed on finite hydrated electron systems. ${ }^{76}$ All these calculations have indicated that the potential energy surface of water cluster anions appears to be very complex and rugged with numerous local minima. This has been illustrated for $\left(\mathrm{H}_{2} \mathrm{O}\right)_{n}^{-}$clusters with $n=6,14,20$ and $24 . .^{71,73,76}$ Due to this complexity, the hydrated electron cluster potential surface is quite sensitive to the choice of the method and the basis set. The best benchmark calculations to date were performed by Herbert and Head-Gordon, showing that at least a large basis $6-3(1+, 3+) \mathrm{G}^{*}$ perturbative MP2 approximation is needed to reach satisfactory accuracy, agreeing with comparable $\operatorname{CCSD}(\mathrm{T})$ calculations. ${ }^{73,74}$ Furthermore, this benchmark showed that DFT results should be interpreted cautiously, since they depend heavily on the employed functional. ${ }^{73} \mathrm{We}$ also note here that the largest cluster size examined to date at the MP2 level, $n=33,{ }^{75}$ is still rather far from reaching the large cluster limit, as will be clear from later discussion.

\subsection{One-electron statistical, finite temperature approaches: adiabatic methods}

In the second group of approaches statistically averaged properties are computed over an ensemble of hydrated electron configurations. These approaches most often apply a mixed 
quantum-classical representation of the system with a subset of degrees of freedom modeled by quantum mechanics, the rest described classically. In the simplest implementation, the quantum mechanically treated excess electron is immersed in a classical solvent bath. The philosophy of the one-electron approach clearly has its origins in the primitive one-electron static models of the hydrated electron. ${ }^{65}$

The first such statistical approach with explicit quantum mechanical treatment for the hydrated electron was based on the Feynman path-integral (PI) theory ${ }^{77}$ formulated within either molecular dynamics (PIMD) ${ }^{78,79,80}$ or Monte Carlo (PIMC) frameworks. ${ }^{81}$ In these path integral simulations of the hydrated electron, only the excess electron is described quantum mechanically by its probability density, and the solvent molecules behave classically. The quantum mechanical distribution of the excess electron is approximated in PI simulations by a necklace of harmonically linked classical beads that interact with the solvent molecules via an electron-water molecule "pseudopotential" (see below). ${ }^{78}$ The great advantage of the PI approach is that the simulations require only classical simulation techniques, and can be relatively easily implemented. Note, however, that path-integral methods have, in general, no correspondence to the temporal nature of the real hydrated electron system, and therefore they are mostly utilized to simulate equilibrium properties. In fact, PI simulations have played an important role in interpreting and reproducing equilibrium properties of hydrated electrons. ${ }^{78-}$ $81,82,83,84,85$ Here we also mention a notable extension, the maximum entropy analytic continuation method of PIMC for the calculation of dynamical properties of the hydrated electron system. ${ }^{86}$

The mixed quantum-classical representation was subsequently implemented to model the time evolution of the hydrated electron using molecular dynamics techniques and wave function representation of the electron. This one-electron mixed quantum-classical molecular dynamics (QCMD) method treats a single electron quantum mechanically, while the solvent 
molecules interact classically and evolve in time according to the classical equations of motion. ${ }^{87,88,89,90,91,92,93}$ Although, the separate treatment of the excess electron from all the other electrons of the system is a strong assumption, the QCMD technique has become historically the most developed and, overall, the most successful theoretical approach up to now to elucidate hydrated electron spectroscopic and dynamic properties. Due to its central role in hydrated electron theory, we summarize relevant technical issues of the QCMD methodology below.

The one-electron QCMD techniques commonly include the following basic elements: an interaction model for the solvent bath, an electron-water molecule pseudopotential describing the interaction between the quantum particle and the classical bath, a representation of the electron, and a prescription on how to evaluate adiabatic electronic states and compute the forces acting upon the solvent molecules. The water molecules are usually modeled by simple classical force fields, such as the non-polarizable, flexible RW2K-M, ${ }^{94}$ and $\mathrm{SPC}^{95}$ models or the polarizable, rigid TIP4P,${ }^{96}$ and AMOEBA potentials. ${ }^{97}$ It is clear that the choice of the water model itself may pose limitations to accuracy (via its ability to reproduce neat water's structural, thermodynamic and dynamic properties). This has been demonstrated for bulk water, ${ }^{98,99}$ neutral water clusters, ${ }^{100}$ and, although to a limited extent, water cluster anions, as well. ${ }^{22}$ Nevertheless, within the mixed QCMD theoretical framework, due to the one-electron approximation, the electron-water molecule pseudopotential appears to be the most critical and sensitive ingredient of the QCMD and path integral simulations of the hydrated electron.

Several electron-water molecule pseudopotentials have been proposed and developed in the literature over the years. ${ }^{78,85,93,101,102,103,104,105,19}$ While the simplest models focused on a water point charge Coulombic model, ${ }^{78,93}$ more sophisticated models ${ }^{19,101-105}$ include exchange in the potential, based on the static exchange (SE) approximation. ${ }^{106}$ In the SE treatment, the 
Schrödinger equation for the excess electron is solved in the field of a single water molecule: ${ }^{101}$

$$
H|\Psi\rangle=\left[T+V_{n}+V_{e}+V_{x}+V_{r}\right]|\Psi\rangle,
$$

where $T$ is the kinetic energy operator, and the potential contains the static nuclear attraction $\left(V_{\mathrm{n}}\right)$, the static electronic Coulomb repulsion $\left(V_{\mathrm{e}}\right)$, and the non-local exchange $\left(V_{\mathrm{x}}\right)$. The repulsion operator $\left(V_{\mathrm{r}}\right)$ in Eq. (1) accounts for the orthogonality of the implicit electrons' wave functions and that of the excess electron, as follows from pseudopotential theory. ${ }^{107}$ The operators are evaluated in the field of the frozen Hartree-Fock wave function of the neutral molecule (SE approximation). In general, two routes in the potential development have been employed within the SE context. The first approach separately approximates the different exact SE interaction contributions (electrostatic, exchange and repulsion) based on welldefined physical models. ${ }^{85,101,102}$ Detailed analysis of the SE components has illustrated, however, that the separate treatment and approximation of the individual components of the SE Hamiltonian introduces large uncertainty in the shape of the pseudopotential in the chemically relevant region after cancellation of large contributions. ${ }^{108}$ This also clarified how numerical inaccuracies in each component of a given pseudopotential ${ }^{109}$ can be fortuitously compensated, leading to a pseudopotential ${ }^{101}$ that overall correctly captures the physical attributes of the hydrated electron. ${ }^{110}$ An alternative approach is to generate an exact pseudowave function of the excess electron in the field of a single electronically frozen water molecule. ${ }^{103,108}$ The pseudo-wave function can be constructed on well-founded grounds ${ }^{107,111}$ and determined numerically in alternative ways. ${ }^{103,112}$ The desired pseudopotential is then optimized within a suitable analytical form to closely reproduce the pseudo-wave function. ${ }^{19,103,104,105}$

Models that employ rigid charge distribution water potentials treat polarization separately, adding a polarization term to the potential a posteriori after the SE treatment. 
$19,85,101,102,103$ This is done using a model potential ${ }^{85,101}$ or by fitting the polarization to some known ab initio potential. ${ }^{102}$ Polarization may also be added, in basically an ad hoc manner, to fine tune the pseudopotential to obtain the agreement with selected experimental data. ${ }^{103}$ Pseudopotentials using polarizable water models introduce polarization in a natural way leading to an iterative, self-consistent procedure: the excess electron distorts the charge distribution of the solvent molecules, and, in return, the solvent's polarized charge distribution influences the excess electron wave function. This aspect was first implemented by Staib and Borgis, ${ }^{93}$ and later developments have built upon the idea. ${ }^{104,105}$ Although all attempts to develop electron-water pseudopotentials on theoretical grounds use the pseudopotential approach, the strikingly different structure of the hydrated electron predicted by these models $^{19,103,104,105}$ and the debate on electron-water pseudopotential development ${ }^{19,113,114,115}$ clearly demonstrates the subtle nature of the challenge. Figure 2 illustrates the problem: the excess electron density computed from the exact pseudo wave function is shown in the field of a single (Hartree-Fock wave function) water molecule and an arbitrary confining potential that forces the electron in the vicinity of the water molecule. Figure 2 shows the projection of the density in the dipole direction of the water molecule in the molecular plane. The electron densities are also computed using two different pseudopotential models where both potentials $^{19,103}$ are based on attempts to reproduce the same exact pseudo-wave function. The relative insensitivity of the fitted electron density for large changes in the parameters leads to significantly different potential surfaces. This difference is manifest in dramatically different hydrated electron structure, as will be illustrated below.

Once all the interactions are known in the system the adiabatic Born-Oppenheimer electronic states are to be evaluated for the electron using efficient numerical techniques. In QCMD approaches the excess electron is directly represented with its wave function that is expanded in either plane waves, ${ }^{87,89,92}$ or distributed Gaussian functions. ${ }^{90,93}$ The plane wave 
expansion is closely associated with the discrete spatial grid representation of the wave function, and the extensive use of Fourier transformation between $r$-space and $k$-space ${ }^{88,92} \mathrm{On}$ the other hand, the use of Gaussian functions significantly simplifies the evaluation of the matrix elements of the Hamiltonian. ${ }^{93}$

The time evolution of the system can be conveniently followed in the wave function representation. In the simplest case, the nuclear degrees of freedom are propagated in time on the adiabatic ground state electronic potential surface. This is generally a good approximation when the energy gap between the ground electronic state and the first excited state remains much larger than typical nuclear thermal energies $\left(k_{\mathrm{B}} T ; k_{\mathrm{B}}\right.$ is Boltzmann's constant). This approach is correct within the Born-Oppenheimer approximation. The time dependence of the electron wave function appears only through the time dependence of the nuclear coordinates on which the electronic wave function is parametrically dependant. In the case of adiabatic (or Born-Oppenheimer) dynamics the force acting upon the water molecules originates from the potential acting only among the water molecules $V_{\text {solv }}$ and from the excess electron according to the Hellman-Feynman theorem:

$$
\underline{F}(t)=-\nabla_{R} V_{s o l v}(\underline{R}(t))+\underline{F}_{Q}(t),
$$

where the quantum force is the expectation value of the negative gradient of the total electronwater molecule pseudopotential $V_{n-e}$,

$$
\underline{F}_{Q}(t)=-\int d \underline{r} \psi_{0}^{*}(\underline{R}(t)) \nabla_{R} V_{n-e}(\underline{r}, \underline{R}(t)) \psi_{0}(\underline{R}(t)) .
$$

The adiabatic ground state wave function $\left(\psi_{0}(\underline{R}(t))\right)$ computed at a given water configuration $\underline{R}(t)$ as the solution of the time-independent Schrödinger equation exerts a force on the water molecules that evolve according to classical dynamics. At the new nuclear configuration the electronic states are again evaluated leading to a sequence of adiabatic time steps. The most important information one receives from QCMD simulations of an excess 
electron in water is the time evolution of the physical properties of the system, such as the eigenvalues of the excess electron (Figure 3). Using such trajectories the adiabatic QCMD simulations are perfectly suited to investigate time-dependent phenomena in equilibrium, including electronic spectra, but may also provide useful information in non-equilibrium scenarios, as well.

In summary, the one-electron QCMD techniques are very useful but do suffer from several shortcomings. The most important limitation of the method is the separate treatment of the excess electron from the other electrons of the bath. This approximation neglects electron correlation and also assumes that the physical attributes of the hydrated electron system arise from elementary events of single electron character. Although this is not strictly

so, as has been demonstrated experimentally, ${ }^{26,42}$ these effects appear to be minor. Within the one-electron picture, the electron-water molecule pseudopotential and the classical water force field limit the accuracy of the theoretical treatment. Most recently a paper overviewed these aspects, and, in particular, compared the capabilities of three different one-electron interaction models in reproducing hydrated electron properties. ${ }^{116}$ Overall, we can conclude that due to their inherent limitations, the one-electron techniques are not quantitative methods. Carefully developed one-electron potentials can be used for, at most, semi-quantitative purposes, such as to reproduce qualitative trends in physical properties of the hydrated electron systems.

\subsection{Extensions of the one-electron adiabatic approach}

A notable attempt to go beyond the single quantum mechanical electron - classical water bath picture is the Drude oscillator model developed by the Jordan group. ${ }^{117,118,119}$ This model is a one-electron model in spirit, but it cleverly extends the quantum mechanical treatment to the electrons of the water molecules with an effective coarse graining procedure. 
The Drude model employs a polarizable water model but replaces the classical polarizable site on each molecule by a quantum Drude oscillator. These quantum oscillators mimic the water electrons and the deformation of the charge cloud of each molecule in the presence of the excess charge and all other water molecules in the system. The Hamiltonian of the excess electron takes into account the electrostatic interactions with the partial charges of the molecules, the induced dipoles, the coupling with the Drude oscillators, and the short-range interaction between the excess charge and the water monomers. The method has been carefully benchmarked to high-level ab initio calculations on small water cluster anions and achieves small deviations in terms of VDE. ${ }^{120,121}$ Nevertheless, its implementation as a computational method to sample phase space is very demanding. The computational effort for the Drude model stems mainly from the multidimensional nature of the Schrödinger equation. Due to its accuracy, this method is very useful to explore the potential energy landscape of smaller clusters, and it also can serve as a benchmark for more approximate methods. ${ }^{119,122,123,124,125}$ In this sense, for larger clusters $(n>\sim 50)$ the Drude model approach, in its present form, still belongs to the static computational approaches discussed in the previous section.

Another straightforward extension of the one-electron methods is the combined approach of one-electron QCMD simulations (to sample the hydrated electron ensemble) and a subsequent higher-level analysis on the configurations collected from the QCMD runs. Several such benchmark calculations have been performed in recent years at the MP2 level. ${ }^{75,126}$ A more complete approach to improve the one-electron model is to treat the excess electron, all (or the valence) electrons of a few molecules nearest the hydrated electron with explicit quantum mechanics, and the rest of the solvent classically. The hydrated electron structure and spectra has been explored along these lines using DFT calculations for the first solvent shell molecules and a point charge model for the remaining solvent. ${ }^{127}$ Calculations of 
the absorption spectrum of the hydrated electron with time-dependent density functional theory have also appeared using QCMD generated configurations. ${ }^{128}$ These calculations assume that the phase space is explored statistically correctly, a hypothesis that can be reasonably tested by comparing the computed physical properties to available experimental data. Such a hybrid cluster anion - classical bath approach was implemented in dynamics by Park et al. ${ }^{129}$ Technical details of this approach are typically a combination of those of the QCMD approaches (above) and the ab initio molecular dynamics techniques described in the following subsection.

\subsection{Ab initio molecular dynamics techniques}

One can extend the quantum chemical treatment to all the electrons of the water molecules (or, at least, all valence electrons, using pseudopotentials) employing $a b$ initio molecular dynamics (AIMD) methodology. Here, the bare nuclei (or the atomic cores) are propagated classically, while the electrons (or valence electrons) are treated by electronic structure methods. Two main procedures that are in general practice for AIMD have been applied to the hydrated electron. The Car-Parrinello molecular dynamics (CPMD) $)^{130}$ technique was used first to investigate the physical properties of hydrated electrons. ${ }^{131,132,133}$ Since CPMD involves fictitious electron dynamics, it is most relevant to evaluation of equilibrium properties. Born-Oppenheimer molecular dynamics (BOMD), where the classical nuclei are explicitly propagated on the Born-Oppenheimer electronic potential surface, is the method of choice when the dynamical details are also under scrutiny. The BOMD technique has been used for adiabatic simulations of excess electrons in finite water clusters. ${ }^{134,135,136,137,138,139,140}$ Due to the many electron character of both CPMD and BOMD, they both currently retain serious size limitations. In fact, it remains unclear if CPMD simulations using periodic simulation cells with 32 molecules faithfully represent the bulk 
hydrated electron system. ${ }^{131,132}$ Cluster BOMD studies ${ }^{134-140}$ are free of the artificial periodicity of bulk simulations, although, as already pointed out, extrapolation to the bulk remains an issue. Nevertheless, the largest water cluster anion simulated so far with AIMD techniques contains an impressive number $(n=105)$ of water molecules. ${ }^{140}$ Technical issues also contribute to uncertainties for AIMD simulations. The typical choice of the electronic structure method is DFT, as all of the cited AIMD studies here have employed DFT. However, as already noted, DFT has well known limitations in accuracy, and, further, cannot be improved in a systematic way, in contrast to wave function-based $a b$ initio methods. Therefore, DFT methods must be independently benchmarked. The most reliable benchmarks for hydrated electron clusters show that DFT methods tend to somewhat overestimate the binding energy of the electron to water, especially in small clusters. ${ }^{73,134}$ A critical element is the choice of the exchange-correlation functional: The Becke-Lee-Yang-Parr (BLYP) functional $^{141,142}$ (in Refs. 131, 132, 133, 137, 138) and the Perdew-Burke-Ernzerhof (PBE) functional $^{143}$ (in Refs. 135, 136, 140), as gradient-corrected approximations to DFT, have been used in hydrated electron simulations. They predict similar liquid water behavior with PBE yielding more stable water clusters. ${ }^{144,145}$ Since the hydrated electron binds relatively weakly to water, the application of a large, very flexible and diffuse basis set is necessary to properly represent the electronic states. Usually, a plane wave basis set is a convenient tool (in Refs. 131, 132, 133), but recently, the hybrid Gaussian and plane wave scheme (GPW) ${ }^{146,147}$ has also been used; the Kohn-Sham orbitals are expanded into a large atom-centered Gaussian basis function in conjunction with an auxiliary plane wave basis (in Refs. 135, 136, 137, 138). The addition of a modest plane wave basis entails the use of soft potentials, and thus necessitates the application of pseudopotentials for atomic cores; the norm-conserving Troullier-Martins $^{148}$ (in Refs. 131, 132, 133, 140) and the Goedecker-Teter-Hutter ${ }^{149}$ (in Refs. $135,136,137,138)$ potentials are used for oxygen core electrons. 
Two other more subtle technical issues further complicate the picture. First, it has been shown that addition of empirical long-range dispersion corrections ${ }^{150}$ improves the description of water. ${ }^{151}$ Most recently, this correction has also been applied in hydrated electron simulations. ${ }^{136,137,138}$ The second problem is due to self-interaction energies in DFT calculations in systems with unpaired electrons, necessarily an issue in hydrated electron simulations, in particular with gradient-corrected approximations (such as with BLYP or PBE functionals). ${ }^{133}$ There are known cases when the self-interaction correction (SIC) ${ }^{152}$ to the DFT functional does not improve the results significantly. ${ }^{132}$ Although SIC has been employed in a number of hydrated electron simulations (mostly in an empirical manner), ${ }^{132,133,135,136,137,138,139}$ it is not yet clear how to optimally parameterize this correction for the hydrated electron. Based on these uncertainties that highlight the potential weaknesses of the DFT methodology in the present context, we conclude that additional rigorous benchmarking of DFT methods in the hydrated electron system is still needed.

\subsection{Non-adiabatic (NA) molecular dynamics methods}

If the Born-Oppenheimer approximation breaks down, adiabatic dynamics is no longer a reliable approximation, and non-adiabatic events (mixing of Born-Oppenheimer electronic states) take place. Such non-adiabatic processes can be represented as electronic transitions of the excess electron between adiabatic states. These typically occur, for example, after large energy excess electrons are created in water, or when non-equilibrium hydrated electron populations are created by interaction with an electromagnetic field (e.g., a pump laser!). The former process of hydrated electron relaxation to equilibrium hydrated electrons and the latter framework for spectroscopic probes of the hydrated electron are both of great interest. The development of simulation techniques taking non-adiabatic electronic transitions into account are considerably more involved both in theory and practical implementation than those 
discussed earlier. So the relatively uncomplicated hydrated electron system is one where several non-adiabatic algorithms treating electronic transitions between adiabatic potential surfaces have been developed and tested as a one-electron QCMD approach. ${ }^{153}$ Since the most important features of these methods have been summarized elsewhere in connection with hydrated electron dynamics, we describe them only very briefly here. ${ }^{154}$

One way to view these methods is from the starting point of the set of (approximate) coupled QCMD Ehrenfest equations of motion. ${ }^{155}$ The time evolution of the excess electronic wave function $(\psi(\underline{r} ; \underline{R}(t)))$ is governed by the time-dependent Schrödinger-equation (TDSE) with the time-dependent potential associated with the nuclear configuration of the solvent bath, $\underline{R}(t)$. The solvent bath, on the other hand, moves classically in the potential of the other water molecules and the electronic Hamiltonian, $\hat{H}_{e}$.

$$
\begin{gathered}
i \hbar \frac{\partial \psi}{\partial t}=-\sum_{i} \frac{\hbar^{2}}{2 m_{e}} \nabla_{r_{i}}^{2} \psi+\hat{V}_{n-e}(\underline{r}, \underline{R}(t)) \psi=H_{e}(\underline{r}, \underline{R}(t)) \psi(\underline{r} ; \underline{R}(t)) \\
M_{I} \underline{R}_{I}=-\nabla_{R_{I}} \int d \underline{r} \psi * \hat{H}_{e} \psi
\end{gathered}
$$

The coupled equations for the electron and the nuclei are to be solved in a selfconsistent manner. The requirement of the consistent time evolution of the quantum and classical degrees of freedom, however, brings up several problems. First, for electronically excited initial states, the time evolution of the nuclear degrees of freedom will eventually lead to trajectory regions where the electronic wave function has become a strong mixture of adiabatic states. This is consistent with the system making non-adiabatic transitions. However, after the trajectory leaves the coupling region, instead of proceeding on a single adiabatic potential surface, the nuclear dynamics will be governed by a weighted average of potential surfaces with weights depending on the history, a result that is not generally theoretically well founded. ${ }^{156}$ The most popular route to restore consistency is via a stochastic 
surface hopping method introduced originally by Tully and Preston, ${ }^{157}$ and later further improved by Tully. ${ }^{158}$ In surface hopping, an ensemble of trajectories is propagated in time with classical nuclear forces determined by the currently occupied quantum basis state. A trajectory may hop to another basis state in a region of strong electronic coupling stochastically according to a probability related to the correct flux between states as determined by integrating the TDSE.

To develop a method with a self-consistent nuclear force, Webster et al. combined the surface hopping method with an expression based on the non-adiabatic theory of Pechukas, ${ }^{159}$ and implemented this technique for the hydrated electron. ${ }^{92,160}$ The advantage of this method (called stationary phase surface hopping (SPSH) method) is that it provides consistent propagation of the classical trajectory and the time-dependent wave function, providing an $a$ priori conservation of energy during hopping events. However, evaluation of the quantum force in the algorithm leads to an iterative self-consistent procedure within each simulation timestep, so that the computational implementation is complex and the computation time is relatively long.

In these QCMD approaches, quantum phase coherences are generally not properly described without specific additional attention, due to the neglect of the role of nuclear wave functions in the QCMD framework. The nuclear wave functions evolving on different electronic potential surfaces quickly diverge and become orthogonal. This divergence causes decoherence, a rapid loss of quantum correlations in the quantum subsystem. ${ }^{161}$ The phenomenon is directly reflected in the decay of the off-diagonal elements of the reduced density matrix of the quantum subsystem (obtained by tracing the density matrix of the full system over the bath coordinates). ${ }^{162,163}$ One approach which integrates quantum decoherence into the QCMD approach and that has been implemented for the hydrated electron is the mean field with surface hopping (MFSH) algorithm. ${ }^{164,165,166,167}$ In MFSH, the calculation of the 
transitions between adiabatic states is determined by the usual stochastic surface hopping principle, but, at the same time, the time evolution of the density matrix incorporates coherence loss based on the current nuclear geometry. Another method, mean-field dynamics with stochastic decoherence (MF-SD) derives the nuclear-induced decoherence rate for the quantum subsystem and uses this rate to determine stochastically whether the system remains in a mixed state or it collapses into one of the adiabatic basis states. ${ }^{154}$ The decay of mixing methods introduce decay terms in the equations of motion for the electronic state population that generate a force component driving the electronic state toward a pure state. ${ }^{168,169,170}$ These methods take decoherence into account in a different way, and therefore predict differences in the non-adiabatic dynamics of the hydrated electron system, as we will illustrate below.

$A b$ initio many-electron simulation techniques have been applied for the hydrated electron most recently to simulate the photoinduced non-adiabatic electron transfer in the hydrated electron system on a $\mathrm{TiO}_{2}$ surface. ${ }^{171}$ This is the only many-electron non-adiabatic study that we are aware of involving the hydrated electron. The method is based on a nonadiabatic dynamics implemented into the time-dependent DFT theory. ${ }^{172}$ The non-adiabatic MD simulations are performed in the mean-field approximation where the TDSE is coupled to the classical nuclear equations of motion (Eqs 4 and 5). In practice, the application of the variational principle to the energy leads to a set of single particle TDSE's for the Kohn-Sham orbitals. The time-dependent one-electron wave functions are expanded in the adiabatic basis, and the coefficient dynamics contains the information on the non-adiabatic transitions. ${ }^{171}$

3.6. Quantum simulations of non-adiabatic events with nuclear quantization: the correlation function approach.

The rate of a non-adiabatic transition might also be evaluated using the standard expression of first-order time-dependent perturbation theory, the Fermi Golden rule. ${ }^{173}$ 


$$
k_{1 \rightarrow 2}=\frac{2 \pi}{\hbar} \sum_{i} \frac{e^{-\beta E_{1 i}}}{Z_{1}} \sum_{f}|\langle 1 i|V| 2 f\rangle|^{2} \delta\left(E_{1 i}-E_{2 f}\right)
$$

Here, the formula gives the thermal transition rate between two adiabatic electronic states, 1 and 2, thermally averaged over the initial nuclear quantum states, $i$, on the initial electronic surface 1 . The delta function ensures energy conservation during the electronic transition ending the system in nuclear quantum states, $f$, on the final electronic surface 2 . In Eq. (6) $V$ is the non-adiabatic coupling operator resulting from the nuclear kinetic energy, while $Z_{1}=\operatorname{Tr}\left(e^{-\beta H_{1}}\right)$ is the canonical partition function $\left(\beta=1 / k_{\mathrm{B}} T\right)$. The golden rule expression can be written in an equivalent time-dependent form ${ }^{174,175,176,177}$

$$
k_{1 \rightarrow 2}=\frac{1}{\hbar^{2}} \int_{-\infty}^{\infty} d t\left[\frac{1}{Z_{1}} \frac{1}{\hbar^{2}} \sum_{i} e^{-\beta E_{1 i}}\left\langle i \mid e^{i H_{1} t / \hbar}\langle 1|V| 2\rangle e^{-i H_{2} t / \hbar}\langle 2|V| 1\rangle i\right\rangle\right]=\int_{-\infty}^{\infty} d t C(t)
$$

where $H_{1}$ and $H_{2}$ are the nuclear Hamiltonians corresponding to the first and second adiabatic electronic states, respectively. Neria and Nitzan proposed a semiclassical evaluation of $C(t)$ approximating the nuclear wave function as the product of frozen Gaussian functions. ${ }^{174,175}$

$$
C(t)=\frac{1}{\hbar^{2}}\left\langle V_{12}^{q-c}(t) V_{21}^{q-c}(0) J(t)\right\rangle_{T}
$$

In Eq (8) $V_{12}^{q-c}(t)$ is the time-dependent electronic coupling, evaluated with classical nuclear momenta, along the classical trajectory propagated on the initial potential surface, and $J(t)$ is a (complex valued) overlap of the two nuclear wave functions, propagating on different potential surfaces. In the practical implementation of the scheme, a number of short trajectories are needed to evaluate $C(t)$. These trajectories are launched from selected configurations of the equilibrium nuclear trajectory on the initial potential surface and are then followed on both the initial and final potential surfaces. The frozen Gaussian based method introduces nuclear quantum effects into the rate calculation scheme a priori, in contrast to the non-adiabatic surface hopping techniques detailed above, but the two methods are intimately connected. ${ }^{176}$ 
An alternative form of the transition rate expression ${ }^{177}$ contains quantum time correlation functions of the non-adiabatic coupling and of the state-to-state energy gap and can be interpreted as integrating the chemical flux correlation function associated with a generalized transport coefficient for the chemical reaction rate. ${ }^{178,179}$ Because of the difficulty in the practice of evaluating quantum correlation functions, a pragmatic approach replaces the quantum correlation functions by their classical counterparts computed from mixed quantumclassical molecular dynamics simulations, with subsequent quantization of the nuclear degrees of freedom using standard a posteriori quantization schemes for classical correlation functions. $^{93,177}$ This quantization procedure can be applied in estimating any physical quantities from mixed quantum-classical simulation techniques that can be derived from autocorrelation functions. One important property, highly relevant to the hydrated electron, is the absorption spectrum for a quantum electronic-bath system given by the well-known Kuboformula $^{180}$

$$
I(\omega)=\frac{\omega}{2 \pi}(1-\exp (-\beta \hbar \omega)) \int_{-\infty}^{\infty} d t \exp (-i \omega t)\langle\hat{\mu} \hat{\mu}(t)\rangle
$$

where $\omega$ is the observation frequency, and $\hat{\mu}(t)$ denotes the time-dependent electronic dipole moment operator. Since Eq. (9) contains the time autocorrelation function of the dipole moment operator (averaged over the electronic eigenstates and the solvent degrees of freedom), the above procedure can be relatively straightforwardly applied for the nuclear quantization procedure of the optical absorption spectrum of the hydrated electron from QCMD simulations. ${ }^{181}$ 


\section{Equilibrium structure and energetics of the hydrated electron}

\subsection{Structural models and energetics of the hydrated electron}

Experimentally, there is no direct probe of the microscopic structure of the hydrated electron. One must turn to theoretical considerations to study the experimental measures and infer the molecular structure that is most closely consistent with experiments. There have been several structural models developed for solvated electrons. The dominant paradigm for the hydrated electron structure is that of the cavity model. ${ }^{58}$ In the cavity model the electron is confined in a solvent void, in a physical cavity, surrounded by properly oriented water molecules. Although the cavity picture has been widely accepted for the hydrated electron, other alternative models have also been proposed. Most recently, a conceptually different model was suggested in which the electron, instead of being confined in a solvent cavity, is delocalized, and extends over many water molecules in a region of enhanced solvent density. ${ }^{19}$ Other alternative models attribute the characteristic hydrated electron properties (in particular, its absorption spectrum, see below) to molecular species other than water molecules. Such species include the hydrated hydronium molecule-hydroxide ion pair $\left(\mathrm{H}_{3} \mathrm{O} \ldots \mathrm{OH}^{-}\right),{ }^{182}$ the solvated solvent-anion complex, ${ }^{183}$ and the hydrated hydronium radical. ${ }^{184,185,186}$ In the following, we overview the experimental observations directly related to the structural aspects of the hydrated electron and confront them with the predictions of various theoretical approaches.

In bulk water, the structural features of the hydrated electron can be most directly deduced from low temperature electron spin resonance measurements. Kevan performed electron spin echo ESR experiments in an aqueous glass at $77 \mathrm{~K} .^{25}$ The experimental signals were interpreted in terms of a cavity type molecular arrangement with six-fold water coordination around the localized electron. The water molecules in the first solvation shell 
were observed to be bond-oriented toward the electron, with the average shortest electronhydrogen distance of $2.1 \AA$. Although this experiment very strongly support the cavity model, it is difficult to evaluate what relevance it may have for ambient experimental conditions. Shkrob performed electron paramagnetic resonance and electron spin echo envelope modulation spectroscopy measurements on hydrated electrons trapped in alkaline glasses, ${ }^{26}$ with results in support of the Kevan model, but without the octahedral regularity. It was also noted that the observed small negative hyperfine coupling constants for protons in hydroxyl groups could not be explained in terms of the simple one-electron model, a result that is not surprising.

Additional important structural information of the hydrated electron can be obtained from the lineshape of its experimental absorption spectrum $(\gamma(\omega)) .^{40} \mathrm{Using}$ spectral moment analysis one can estimate the thermal dispersion and the kinetic energy of the excess electron: $183,187,188$

$$
\begin{aligned}
& M(-1)=n_{0} \int_{0}^{\infty} \omega^{-1} \gamma(\omega) d \omega=\frac{2}{3}\left\langle r^{2}\right\rangle \\
& M(0)=n_{0} \int_{0}^{\infty} \gamma(\omega) d \omega=1 \\
& M(1)=n_{0} \int_{0}^{\infty} \omega \gamma(\omega) d \omega=\frac{4}{3}\langle T\rangle,
\end{aligned}
$$

where $n_{0}$ is the refractive index of the solvent, $\left\langle r^{2}\right\rangle$ and $\langle T\rangle$ are the square of the radius of gyration and the kinetic energy of the electron. One notes that an important assumption behind the use of the spectral moments is that the coupling between the excess electron and the other electrons of the system is weak. ${ }^{183,187}$ This assumption is consistent with the use of one-electron computational models. The radius of gyration $\left(\left\langle r^{2}\right\rangle^{1 / 2}\right)$, characterizing the size of the hydrated electron, has been determined and described by an empirical series expansion in temperature, giving $2.45 \AA$ at ambient conditions. ${ }^{188}$ 
A realistic theoretical description of the structure of the bulk hydrated electron requires statistical models using either one-electron or simplified many electron quantum techniques. Computational limitations restrict static quantum chemical calculations to the reliable characterization of the potential energy surfaces of small hydrated electron clusters. In addition to the interest in clusters per se and as possible windows on the bulk, quantum chemistry offers precise benchmarks for statistical methods which use strong approximations. Hence, we overview the results of quantum mechanical calculations in the cluster section below.

Path-integral and mixed quantum-classical molecular dynamics simulations have been performed for the bulk hydrated electron within the context of the one-electron model. The simulations, from the first pioneering PIMC $^{85}$ and PIMD $^{78,79}$ studies and one-electron QCMD simulations ${ }^{88,93}$ to more recent attempts, ${ }^{103,105}$ predominantly support the cavity model for the hydrated electron. One-electron MD simulations ${ }^{88}$ predict that the approximately spherical, $s$ type ground state is followed by three non-degenerate $p$-like excited states, with degeneracy broken by the fluctuating solvent environment. The solvent fluctuations influence the size and the shape of the electron cavity and, as a result, act on the energy levels of all states. These factors have an important role in determining the shape of the absorption spectrum of the hydrated electron, as will be illustrated below. Table 1 collects selected simulated structural parameters for alternative measures and models. The radius of gyration varies in the 2.1-2.7 range (see Table 1). The solvent structure is characterized by radial distribution functions from the center of the electron distribution to the hydrogen and oxygen sites of the water molecules. The distribution functions testify that water molecules are predominantly bond oriented with the hydrogen atoms pointing toward the electron. The coordination numbers in the first coordination sphere around the electron change from 4 to 6 depending on the particular model. 
Most recently an alternative electron-water molecule pseudopotential has been developed that predicts non-cavity structure for the solvated electron in QCMD simulations. ${ }^{19}$ The excess electron in this model becomes delocalized over several water molecules in a region where the solvent density is increased relative to that of neat water. The best illustration of the cavity vs. non-cavity character is seen in the electron-hydrogen and electron-oxygen radial distribution functions; see Fig. 4. In the cavity model (top panel), a distinct excluded volume appears around the origin where the center of the electron is located, while in the non-cavity model (bottom panel) no such feature is apparent on the distribution functions. Another pictorial representation of the main features of the cavity and the noncavity models is shown in Figure 5 with the electronic isodensity surfaces corresponding to the $80 \%$ probability of finding for the excess electron. The non-cavity model has immediately been seriously criticized. ${ }^{113,114}$ In particular, it was pointed out that a) the model's predictions are not consistent with high level ab initio results, ${ }^{114}$ and b) that the fitted non-cavity potential appears to distort important parts of the exact pseudopotential. ${ }^{113}$ This distortion leads to an artificial decrease of the electron density on the hydrogen side and an artificial enhancement of the electron density on the oxygen side of the water molecule. ${ }^{113}$ A comparison of the structural parameters of the non-cavity model with experiment and with other models reveals that the non-cavity model generally predicts a more diffuse electron distribution with a radius of $2.69 \AA .^{116}$

The only many-electron quantum molecular-dynamics studies that have been performed on bulk hydrated electron are the CPMD simulations on a small $(n=32)$ periodic sample of water molecules by Boero et al. ${ }^{131,132}$ The calculations are based on DFT electronic structure calculations using the Becke-Lee-Yang-Parr functional. ${ }^{141,142}$ The simulations predict that the excess electron localizes in solvent cavities with characteristics similar to the cavity state just described. The cavities appear to have a short lifetime of 50-90 fs. The radius 
of the highest occupied Kohn-Sham molecular orbital is computed to be $2.2 \AA$, shorter than, but close to, the electron radius in one-electron simulations.

The perturbation of the water matrix by the hydrated species has been investigated by Tauber and Mathies performing resonance Raman spectroscopy experiments on the hydrated electron. ${ }^{57}$ Their vibrational spectra manifest significantly downshifted $\mathrm{HOH}$ bending (by $~ 30$ $\mathrm{cm}^{-1}$ ) and $\mathrm{OH}$ stretching vibrations (by $\sim 200 \mathrm{~cm}^{-1}$ ) strongly suggesting that the resonance Raman scattering of the aqueous solvated electron originates only from perturbed water molecules. These results support those models for the hydrated electron that do not contain other water containing species besides molecular water. The study emphasizes that the solvated anionic cluster model of the hydrated electron ${ }^{19}$ and the cavity $\operatorname{model}^{58}$ as a special case of the solvated anion picture are consistent with the measurements. This has been tentatively shown to hold true for both one-electron non-cavity ${ }^{19}$ and cavity models. ${ }^{105}$

The most fundamental energetic data of the hydrated electron is its VDE. Although experimental VDE values for finite size hydrated electron clusters have been available since the 1990 's, ${ }^{46}$ the direct measurement of the bulk VDE is very recent. Time-resolved photoelectron spectroscopy of the charge-transfer-to-solvent reaction in aqueous KI solutions have resulted in a value of $3.27 \pm 0.10 \mathrm{eV}$ for the bulk hydrated electron VDE. ${ }^{189}$ Similar measurements predicted comparable values with slight variation, $3.3 \mathrm{eV}^{18}$ and $3.6 \mathrm{eV} .^{55}$ The interpretation of the simulated bulk VDE values is complicated by the fact that there are technical difficulties associated with the implementation of the Ewald summation in QCMD simulations. ${ }^{93,105,190}$ It has been shown that the effect of inclusion of long-range corrections in simulations with periodic boundary conditions can be significant; the application of Ewald summation in a hydrated electron QCMD simulation shifts the VDE considerably, from the non-corrected $3.1 \mathrm{eV}$ to $3.9 \mathrm{eV} \cdot{ }^{190}$ Further analysis pointed out that this corrected result is the consequence of cancellation errors from neglecting polarization and finite box-size effects. ${ }^{105}$ 
It was also pointed out that the simulated geometric parameters were nonetheless insensitive to the long-range correction. ${ }^{103,190}$ Ewald corrected VDE values of $\sim 3.2 \mathrm{eV}^{93}$ and $3.7 \mathrm{eV}^{105}$ were reported using polarizable water force fields in good agreement with experiments. The VDE computed from the most recent non-cavity forming pseudopotential is significantly larger than these values, $\sim 5-6 \mathrm{eV} .{ }^{19}$ Another way to obtain the bulk hydrated electron VDE is from extrapolating cluster anion VDE values to the infinite size limiting case. We review this aspect in the cluster context, below.

\subsection{Structure and energetics of water cluster anions}

Finite size hydrated electron systems (clusters, interfacial hydrated electrons) have added significantly to the complexity of the hydrated electron structural picture, a fact not anticipated in the early experimental observations of hydrated electron clusters $\left(\left(\mathrm{H}_{2} \mathrm{O}\right)_{n}^{-}\right) .^{43,44,45}$ Experimental works on water cluster anions have been strongly inspired in part by pioneering simulations of Barnett et al. ${ }^{80,82,83,191}$ that first predicted the appearance of two distinct types of cluster configurations which should be distinguishable experimentally. The first group of configurations binds the excess electron in a cavity type arrangement, sometimes called an interior state; such clusters can be viewed as embryonic hydrated electron systems. Alternatively, the excess electron could be attached to the surface of the water cluster with the excess electron density localizing mainly outside the molecular frame of the cluster. These clusters are usually called surface state clusters, or sometimes dipolebound clusters. The connection of these two types of water cluster anion isomers to infinite size extrapolated hydrated electron systems is illustrated in Figure 6. Increasing the size of interior state water cluster anions extrapolates to the bulk hydrated electron, while the same limit for surface state cluster anions is a surface hydrated electron at an infinite water/air interface. 
The photoelectron ${ }^{46,48}$ and photodestruction spectra ${ }^{47,192}$ of various sized hydrated electron clusters indicated smooth evolution of the data (VDE and absorption spectra) with size with no apparent break. More precisely, a linear relationship was observed in $\left(\mathrm{H}_{2} \mathrm{O}\right)_{n}^{-}$ clusters between cluster VDE and $n^{-1 / 3}, 46$ in agreement with the prediction of dielectric continuum theory. ${ }^{66,83}$ This observation and lineshape fitting of the absorption spectra led Coe et al. to interpret the data as belonging to a single structural motif, assigning this motif to interior state water cluster anions. ${ }^{46,193}$ Extrapolation of the cluster data to infinite size was taken as the VDE of the bulk hydrated electron $(3.3 \mathrm{eV}) .{ }^{46}$ This data agrees well with the results of recent direct VDE measurements for the bulk hydrated electron of $3.27 \pm 0.10$ $\mathrm{eV},{ }^{189} 3.3 \mathrm{eV}^{18}$ and $3.6 \mathrm{eV} .^{55}$ Nevertheless, spectral moment analysis of the optical absorption lineshape for various size clusters indicated that the evolution of the kinetic energy and the radius of the hydrated electron with size might be consistent with alternative assignments for the hydrated electron structural isomers. ${ }^{187}$ More recent cluster experiments ${ }^{13,17,194,195,196}$ revealed the existence of at least three different trends of the VDE with increasing cluster size. In particular, the Neumark group observed three distinctly different tendencies, ${ }^{13}$ while the photoelectron spectra of cold size selected water cluster anions indicated that the strongest bound feature of the photoelectron spectra of cold water cluster anions (thermalized at $10 \mathrm{~K}$ ) possess three distinct components. ${ }^{196}$ The appearance of several different patterns in the photoelectron spectra shows that the structural description is at least more complex than simply interior state vs. surface state clusters. In addition, the VDE of surface hydrated electrons at infinite water/air interface, the limiting case of surface state hydrated electron clusters, has been reported most recently from ultrafast liquid jet photoelectron spectroscopy measurements. ${ }^{18}$ This VDE, $1.6 \mathrm{eV}$, is significantly smaller than that for the bulk hydrated electron. We note, however, that several subsequent liquid jet studies were unable to confirm that this signal belongs to long-lived surface hydrated electrons. ${ }^{54,55,56}$ 
A different facet of the problem, the molecular details of the electron-water molecule interaction were interrogated in small $(n<7)$ clusters by Johnson and his group probing argon-solvated water cluster anions via infrared spectroscopy. ${ }^{12,197}$ The results point to an important role of a water molecule that binds the electron with both $\mathrm{OH}$ groups, so that is connected to the cluster hydrogen-bonded network with only a double hydrogen-bonding acceptor (AA) motif. The spectral signature identified in smaller clusters is found to be important in the intermediate size regime $(n=7-21)$, as well. ${ }^{198}$ As the cluster size further increases, the bending feature characteristic of the AA motif remains present, but shifts to the blue and broadens. This was interpreted as an indication that other water molecules increasingly participate in stabilizing the electron, and the electron binding motif becomes more delocalized. ${ }^{199}$ We note that in this latter experiment the temperature of the ion trap was held at $20 \mathrm{~K}$ defining the temperature of the clusters more reliably than in previous experiments.

As we indicated in the previous section, several static quantum chemistry computational studies on small size water cluster anions have been performed with geometry optimization..$^{71,72,73,74,76}$ The calculations indicated that the potential surface of even very small water cluster anions is very complex with a large number of local minima. Of the minima, most configurations support the excess electron density mainly concentrated on the surface of the cluster, while a relatively few exhibit interior state character. The relative energies of these local minima, however, strongly depend on the computational level and basis set. While $a b$ initio calculations on the smallest optimized static cluster anion structures $(n \leq 6)$ have been used to reliably assign the experimental photoelectron spectra, ${ }^{73}$ due to the increasing complexity of the potential surface with size, there is still no consensus for larger clusters. The best available ab initio calculations for medium sized clusters at scaled MP2/6$31(1+, 3+) \mathrm{G}^{*}$ level show the presence of several weakly bound and strongly bound isomers in 
$\left(\mathrm{H}_{2} \mathrm{O}\right)_{20}^{-}$and $\left(\mathrm{H}_{2} \mathrm{O}\right)_{24}^{-}$clusters. ${ }^{73}$ The excess electron distribution of the isomers indicates either interior or surface excess electron type ${ }^{73}$ via natural bond orbital analysis ${ }^{200}$ of singly occupied molecular orbitals. This limited cluster size is in any case not sufficient to anticipate the behavior of larger clusters, particularly under finite temperature experimental conditions. This is especially true when one selects and analyzes only a few local minimum configurations for analysis, ${ }^{76}$ as was clearly pointed out by Sommerfeld. ${ }^{201}$

Quantum chemistry cluster calculations also provide an important insight into the electronic origin of the large experimental vibrational shifts in hydrated electron systems. ${ }^{57}$ Large basis set DFT calculations, vibrational and natural bond orbital analyses indicated significant charge penetration from the excess electron to the $\mathrm{O}-\mathrm{H} \sigma^{*}$ orbital on a water molecule with double hydrogen bond acceptor (AA) motif in small water cluster anions $(n=4,6) .{ }^{202}$ This charge penetration is shown to lead to sizable O-H vibrational red-shifts. An embedded cluster study at DFT/BLYP level on large interior state water cluster anions taken from bulk QCMD simulations resulted in similar conclusions. ${ }^{127}$ Here, a significant transfer of the unpaired electron's density is observed to the $2 \mathrm{p}$ oxygen orbital of the $\mathrm{OH}$ groups nearest the electron. Although both studies conclude that excess charge penetration to water unoccupied orbitals is a many-electron phenomenon that cannot be captured in the strict oneelectron models, the overall density transfer is minor, estimated to be less than roughly 20 $\% .^{127}$

The Drude-model of the Jordan group, ${ }^{117-125}$ discussed already, is relatively expensive in large size simulations, but has been employed with success in parallel tempering Monte Carlo simulations to map the potential surface of finite temperature small water clusters. ${ }^{119,122,124,125}$ For the $n=12-24$ range, they identified three different binding motifs, namely, surface states, cavity states and a hydrogen-bonding network permeating pattern. 
Possible surface states also include those with double hydrogen-bonding acceptor water molecules. $^{123}$

Despite the deficiencies of one-electron models discussed in previous sections, the clear advantage is that all cluster sizes observed in experiments can be readily simulated using this technique. There have been several attempts to simulate water cluster anions with oneelectron models. The first series of works came from the Landman group. These works addressed a wide variety of anionic cluster problems: electron localization in clusters, ${ }^{80,83,102}$ spectroscopy of excess electrons in water clusters ${ }^{89,203}$ and various dynamics investigations of electron excitation, ${ }^{82}$ relaxation dynamics ${ }^{204}$ and non-adiabatic processes. ${ }^{174,175}$ The next wave of systematic cluster anion investigations, using a different electron-water molecule pseudopotential, ${ }^{103,108}$ was not launched until 15 years later. ${ }^{14,113,126,190,205}$ The results parallel each other where they can be compared, but they are for the most part complimentary. Most recently, a cluster simulation study using a polarizable electron-water pseudopotential was published. ${ }^{206}$ Here, we review the energetic and structural questions, and postpone discussion of spectroscopy and dynamics to later sections.

The PIMD study of Barnett et al. on $\left(\mathrm{H}_{2} \mathrm{O}\right)_{n}^{-}$clusters with $n=8-128$ predicted the existence of interior and surface state excess electronic states. ${ }^{83}$ More recent QCMD studies extended the simulated cluster size range up to 8000 molecules. ${ }^{14,190}$ It was found that the localization of the excess electron depends on the size and temperature (internal energy) of the clusters. In general, smaller clusters tend to stabilize in surface states, while larger clusters prefer interior state electron localization, as expected from the earlier studies. ${ }^{83}$ More importantly, the different electron localization modes, surface vs. interior localization, lead to clearly different physical observables. The observed trends can be directly compared to available photoelectron data and used to identify common structural patterns within the groups. These issues were also demonstrated in the latest cluster anion QCMD simulations. ${ }^{206}$ 
The first area for comparison concerns structure. QCMD simulations easily distinguish surface state structures and interior state clusters by their geometric parameters, readily accessible from simulations. For surface states, the distance $(R)$ between the centers of the electron distribution and the center of mass of the cluster is comparable to the radius of the cluster $\left(r_{\mathrm{c}}\right), R \sim r_{c}$, while for interior states $R+r_{\mathrm{e}}<r_{\mathrm{c}}$, where $r_{\mathrm{e}}$ is the electron radius (radius of gyration). The size dependence of the simulated electron radius exhibits characteristic tendencies for surface state and interior state clusters. ${ }^{14}$ Figure 7 shows the computed radius (and the associated kinetic energy) of the excess electron in varying size interior state and surface state clusters, as computed by Madarász et al. ${ }^{190}$ The radius is nearly invariant (2.3$2.4 \AA$ ) in the simulated interior state clusters and is in good agreement with the bulk hydrated electron radius $(2.4 \AA) .{ }^{25}$ The surface bound states are distinctly more diffuse, contract upon increasing the cluster size and smoothly decrease to the radius of a hydrated electron on an infinite water/air interface (see Figure 6 and the discussion below). The simulated behavior can be compared with the trend for the experimental radii extracted from the photodestruction data of Ayotte and Johnson ${ }^{47}$ by Bartels using spectral moment analysis. ${ }^{187}$ The analyzed experimental PES data also closely corresponds to the clusters measured in the Coe et al. experiments. ${ }^{46,193}$ Simulations found ${ }^{14,190}$ that the simulated trend for the surface state radii parallel that derived ${ }^{187}$ from the available experimental action spectra. ${ }^{47}$ This trend was also observed in the Barnett simulations, ${ }^{83}$ although both sets of simulations overemphasize the diffuse nature of the electron in small size clusters. We note that a similar size dependence of the electron radius in surface state clusters is not fully evident in the latest study, by Jacobson and Herbert. ${ }^{206}$ As expected, for data that shows a parallel between moment analysis and simulated radii, a similar pattern holds for the kinetic energy of the excess electron, ${ }^{14,190}$ also shown in Figure 7. The cavity states have higher kinetic energy than the surface states, and all interior state kinetic energies are similar to one another. The kinetic energy increases with 
increasing cluster size for surface states approaching the bulk interface limit, now from below. This trend follows the experimentally derived size dependence. ${ }^{187}$

Turning to the energetics of water cluster anions, the top panel of Figure 8 collects the most relevant experimental VDE data showing six distinct sets. The variation in the experimental data originates from different experimental conditions. In particular, varying the backing pressure of the carrier gas in the cluster preparation process significantly influences the experimental observations. ${ }^{13,207}$ It is generally assumed that higher pressure corresponds to lower cluster internal energies, that is, colder clusters, indicating that cluster internal energy plays a key role in the physics of molecular cluster anions. The most strongly bound feature of Neumark's photoelectron spectra, most prominent at the warmest conditions (denoted as type I clusters), were attributed to interior bound cluster anions, while the other two (type II and III) were assumed to be surface state clusters. ${ }^{13,17,195,208,209}$ The observed dynamics of the hydrated electron clusters are also explained in terms of this picture (see below). ${ }^{13,210,211} \mathrm{We}$ note that cluster type I VDE's correspond closely to those observed by Coe et al. ${ }^{46,193}$ Three distinct components of the strongest binding motif of size selected water cluster anions were identified by Ma et al. in the photoelectron spectra of cold water cluster anions. ${ }^{196}$ The position of the peaks for Ia and $\mathrm{Ib}$, the two dominant species brackets those for type I of Neumark. The fact that the ratio of the offset energy of the Ib peak to the main peak (Ia) with water and heavy water rapidly converges to 1 indicates that the new Ib feature is likely not of vibrational, but of structural origin. Class Ic has also been observed, but with large uncertainty and were tentatively assigned to the vibrational excitation of class Ib. ${ }^{196}$ Ma et al. considered the structural differences between the dominant Ia and Ib isomers, and raised the possibility that group Ib contains water cluster anions with internally localized excess electron. In fact, observing magic numbers in the mass spectra of these data suggests interior state clusters in group Ib. ${ }^{196}$ 
The most important simulated VDE results are collected in the bottom panel of Figure 8. Although the results differ in the magnitude of the VDE, the main tendencies of the experimental and simulated data are shared. Interior state simulations predict stronger binding than those for surface stabilized electrons, and the interaction strength increases with cluster size. Of the different simulations, Barnett et al. predict by far the strongest binding for the interior bound isomers, much stronger than any observed VDE data (not shown) ${ }^{83}$ Their surface state VDE values are also the largest among simulations. The interior state simulated data of Madarász et al. at a nominal temperature of $T \sim 200 \mathrm{~K}$ have a large size limit intercept of $4.4 \mathrm{eV}^{190}$ and correspond reasonably to the strongest experimental features of Ma et al., of which group $\mathrm{Ib}$ is tentatively assigned to consist of interior state isomers (with a $4.0 \mathrm{eV}$ intercept $).{ }^{196}$ The latest, Jacobson-Herbert interior state simulations $(T=200 \mathrm{~K})$ are also in the same range, although a bit less stable, with a $3.4 \mathrm{eV}$ bulk extrapolation. ${ }^{206}$ The experimental feature, I, has been argued by experimentalists for a while as consisting of interior state clusters. $^{13,17,46,193,195}$ The patterns of the electron radius with size as identified in spectral moment analysis ${ }^{187}$ and in simulations, ${ }^{14,83,190}$ however, indicate surface state character for type I. The equilibrated surface state clusters of Madarász et al. also fall in the same VDE regime, but show distinct deviation from linearity at large cluster sizes. ${ }^{190}$ Modest cluster sizes $(n \leq 200)$ of the surface series extrapolate to $\sim 3 \mathrm{eV}$ reasonably close to that obtained by experiments, ${ }^{13,17,46,193}$ while deviations from linearity push the limiting value at an infinite interface to a considerably more positive value, $\sim 4 \mathrm{eV}$. Jacobson and Herbert considered three types of surface states for water cluster anions: dipole-bound clusters, proper surface isomers and partially embedded surface clusters. ${ }^{206}$ Since these isomers may appear within the same trajectory, and the clusters may also fluctuate between these forms, their clear distinction is not without uncertainty. Nevertheless, the partially embedded structures at $T=$ $100 \mathrm{~K}$ coincide well ( $3.3 \mathrm{eV}$ intercept) with experimental type I cluster. However, they don't 
exhibit the size dependence of the electron radius inferred from measured spectral moments. ${ }^{187}$ The proper surface isomers (found in $T=100 \mathrm{~K}$ and $200 \mathrm{~K}$ simulations) extrapolate to smaller VDE, $2.4 \mathrm{eV}$, somewhere between the experimental types of I and II (see Figure 8). Although this isomer shows some indication of change in the electron radius of gyration with size, this effect is still modest. ${ }^{206}$ For comparison, in the bottom panel of Figure 8 , we have added the predictions of a dielectric continuum treatment ${ }^{190}$ based on the work of Makov and Nitzan. ${ }^{66}$ We find it striking that this simple theory of surface states also provides reasonable VDE predictions. Nevertheless, we point out that all simulated infinite size extrapolated surface clusters have significantly larger VDE than reported experimentally, 1.6 $\mathrm{eV}^{18}$ a discrepancy that should be addressed and resolved in the future.

The key point in understanding the experimental VDE signatures is the cluster preparation protocol. In fact, the alternative groups of simulated data, in particular those of Jacobson and Herbert, ${ }^{206}$ simply reflect different initial simulation conditions. Cluster anions can be trapped to different metastable states depending on the initial conditions. The most obvious choice to manipulate cluster preparation is by varying the simulation temperature or the initial preformed cluster structure. A recent systematic simulation study modeled the influence of cluster preparation procedure on the observable physical properties of water cluster anions. ${ }^{205}$ Two protocols have been designed for preparing neutral water clusters to which one adds an excess electron at the beginning of the simulation. In one (so called quenched clusters), neutral equilibrium clusters are prepared at relatively high temperature, and then quenched down to the desired low temperature. In the second one (metastable clusters), the clusters are prepared at very low temperature (near $0 \mathrm{~K}$ ), and slowly heated up to the desired temperature. The incomplete relaxation of the two types of clusters at low temperature leads to different observed properties, including the VDE distribution, up to 200 $\mathrm{K}$ when these properties slowly converge. 
The few available many electron dynamics calculations provide important additional insight. In the smallest size regime, Herbert and Head-Gordon showed quantitative agreement of the simulated and experimental vibrational spectra for a hydrated electron tetramer. ${ }^{134}$ The double acceptor (AA) binding motif was found to be very stable to thermal fluctuations. Jungwirth et al. performed AIMD simulations on medium-sized $(n=32)$ water cluster anion. At this size, the simulations suggest that the excess electron is predominantly located at the surface of the cluster. The binding energy strongly correlates with the size (radius) of the excess electron, indicating that photoelectron spectroscopy can determine, via this correlation, the size of the excess electron (see Figure 9). ${ }^{135,139}$ Similar correlation was also found by Jacobson and Herbert using one-electron simulations but also extended to multiple cluster sizes and different binding motifs. ${ }^{206}$ The strong correlation indicates universal relationship that was also analyzed in Ref. 206 using a simple theoretical model. Interestingly, while no apparent correlation was found between VDE and the relative position of the electron to the cluster in AIMD simulations, ${ }^{139}$ the Jacobson-Herbert one-electron simulations indicate a fairly strong correlation. ${ }^{206}$ The VDE distribution of Jungwirth et al. along a single AIMD relaxation trajectory exhibits two peaks, at $\sim 0.7 \mathrm{eV}$ and $\sim 1.6 \mathrm{eV},{ }^{135,139}$ in good agreement with the corresponding experimental points for type II and I clusters, ${ }^{13}$ correspondingly. The average radius of gyration, $\sim 3 \AA$ also corresponds well with experiments. Computer simulation of the cluster preparation procedure adding an excess electron to neutral clusters was revisited by investigating cluster relaxation of the same $n=32$ size cluster at $T=20 \mathrm{~K}, 50$ $\mathrm{K}$ and $300 \mathrm{~K} \cdot{ }^{138,139}$ In another scenario, an electron was attached to an equilibrated neutral water cluster at $T=300 \mathrm{~K}$, then instantaneously quenched to $\sim 0 \mathrm{~K} .{ }^{137}$ In both cases, the observation of metastable states challenged the validity of extrapolation of solvated electron properties from cold clusters to bulk liquid. ${ }^{137,139}$ The most recent AIMD simulations were performed by Barnett et al. ${ }^{140}$ The simulation employs DFT with the PBE exchange- 
correlation functional and plane-wave basis set. ${ }^{143}$ This AIMD study has examined the broadest range of hydrated electron clusters with $n=15-105$ at $T=250 \mathrm{~K}$. Three isomers were identified, diffuse, surface and interior states. The VDE values for the diffuse state correspond nicely to type II clusters, while small and medium size surface state clusters and medium size interior clusters reproduce type I trends well (see Figure 8). Surface and interior state clusters have basically the same calculated VDE for the largest examined sizes, $n=85$ and 105. We note, however, that although it has been suggested that gradient-corrected methods are suspected to suffer from self-interaction error and overestimate VDE, ${ }^{133,135}$ this study did not include SIC. Thus, although this study unquestionably represents an example of the next generation of methodology for these systems, ${ }^{140}$ the assignment of several experimental features remains unclear. In addition, without a notable extension of capabilities, AIMD calculations remain unable to address the extrapolation of cluster properties to those for the bulk hydrated electron species.

Direct comparisons of bulk simulation VDE to extrapolated cluster properties are scarce. The main difficulty here is, as discussed above, that the comparison is valid only if the simulation conditions, most notably the temperature, of clusters and the bulk are at least comparable. The extrapolation of the cluster data by Jacobson and Herbert results in $3.4 \mathrm{eV}^{206}$ for $T=200 \mathrm{~K}$ interior state clusters, in reasonably good agreement with their Ewald corrected bulk simulation data $(3.7 \mathrm{eV})$ at $T=300 \mathrm{~K} .{ }^{105}$ The bulk, room temperature simulation using the pseudopotential of Turi and Borgis ${ }^{103}$ with Ewald correction $(3.9 \mathrm{eV})$ underestimates the corresponding extrapolated VDE at $200 \mathrm{~K}$ by $0.5 \mathrm{eV} \cdot{ }^{190}$ Simulations of a surface bound hydrated electron on an infinite water/air interfaces would provide the comparison for the extrapolated surface state cluster VDE. Interfacial surface state simulations have been performed using both one-electron $\mathrm{QCMD}^{212}$ and many-electron CPMD techniques. ${ }^{133}$ Surface state hydrated electrons on an infinite supercooled water/air interface (200 K), Ih 
ice/air interface $(200 \mathrm{~K})$ and an infinite amorphous solid water/air interface $(100 \mathrm{~K})$ yield VDEs, respectively, of $2.6 \mathrm{eV}, 2.7 \mathrm{eV}$ and $1.6 \mathrm{eV}^{212}$ without the Ewald summation and using the Turi-Borgis pseudopotential. ${ }^{103}$ Although it is difficult to compare these data to the extrapolated cluster results, $0.8 \mathrm{eV}$ extra stabilization from long-range contributions and allowing for a $0.5 \mathrm{eV}$ underestimation relative to the extrapolated cluster data in the bulk case would suggest again reasonable agreement between bulk and cluster data. In fact, Rodriguez and Laria simulated artificial surface bound electrons at $298 \mathrm{~K}^{213}$ using the same pseudopotential $^{103}$ with Ewald correction. They found $-3.5 \mathrm{eV}$ ground state energy, in line with the above estimate and in support of the extrapolation procedure. ${ }^{213}$ These simulated surface VDE data once again are greater than the experimentally suggested VDE value of 1.6 $\mathrm{eV}^{18}$

Structurally, the relaxed surface excess electron states in the interface QCMD simulations at $200 \mathrm{~K}$ are clearly seen to partly penetrate into the condensed phase, and in this sense they are similar to what has later been termed partially embedded structures by Jacobson and Herbert. ${ }^{206}$ The electron radius turns out to be somewhat larger, $2.6 \AA,^{212}$ than that in the bulk $(2.45 \AA),{ }^{188}$ in agreement with experimental observations. ${ }^{187}$ Minimal reorganization of the water in a $100 \mathrm{~K}$ simulation at a solid amorphous ice/air interface allows essentially no penetration, resulting in a larger electron radius, $3.0 \AA \AA^{212}$ The only AIMD study on surface hydrated electrons, by Baletto et al., simulated surface trapped electrons on Ih ice/air interface, but neither the VDE nor the radius were reported. ${ }^{133}$ 


\section{The equilibrium optical absorption spectrum of the hydrated electron}

Its optical absorption spectrum was the principal means of identification in the original observation of the hydrated electron. ${ }^{1}$ The hydrated electron spectrum, a broad, featureless, asymmetric band with a maximum at $1.72 \mathrm{eV}$, was measured and characterized in great detail by Jou and Freeman. ${ }^{40}$ From a theoretical point of view, to reproduce and explain the shape of the absorption spectrum has proven to be a nagging challenge. Since the optical absorption spectrum appears to be a characteristic signature of the hydrated electron, it is often used in theoretical approaches to a posteriori justify the validity of the model on which the calculation is based. Unfortunately, the hydrated electron's essentially featureless absorption band can be described by basically different models with similar success. With the most recent development of new pseudopotential models and the increasing availability of AIMD techniques, the problem now seems to have converged to a satisfactory resolution. In this section we will give a short review of the progress of the theory of the hydrated electron absorption spectrum.

Early theoretical models based on ad hoc assumptions have been used to rationalize the hydrated electron spectrum, but these attempts remained largely unsuccessful and failed to provide a firm theoretical basis for the absorption line shape. ${ }^{65,214}$ The first successful attempts to simulate the absorption spectrum and suggest a detailed description of the physics underlying the optical spectrum were based on one-electron models. A pioneering simulation study came from Rossky, Friesner, and coworkers. ${ }^{87}$ The absorption spectrum envelope was constructed from instantaneous values accumulated from an ensemble of electronic ground state configurations generated via PIMD simulation. The basic features of the computed spectrum are common to all later work. It is generally accepted that three electronic transitions dominate the spectrum, from the s-type electronic ground state to the first three, 
non-degenerate, p-states. ${ }^{88}$ It was also pointed out that fluctuations of the solvent cavity radius influence the mean s-p energy gap, while fluctuations from spherical symmetry (asymmetric distortions) modulate the splitting of the p-levels. The inhomogeneous broadening of the spectrum was thus attributed roughly equally to solvent fluctuations in the size and the shape of the solvent cavity. ${ }^{88}$ The most important simulated features of the computed spectrum are shown in Figure 10. The simulated absorption spectrum however was not able to correctly describe the position and the shape of the spectrum. The maximum is $\sim 0.7 \mathrm{eV}$ shifted to the blue, while the half-width is underestimated. A related problem is that the intensity of the blue-side of the spectrum, the high energy tail is not reproduced with acceptable accuracy. ${ }^{88}$ This study, although inadequate in some quantitative respects, nevertheless shed light on fundamental qualitative aspects underlying the origin of the spectrum. ${ }^{88}$

The failure to correctly reproduce the experimental spectrum in simulations has two major, related origins: the application of pseudopotential models and the neglect of the quantum behavior of the solvent bath in the simulations (see later). The most important source of discrepancy between experiment and theory likely originates from the approximate nature of the employed pseudopotentials especially in predicting the energetics of the excited states. This problem is characteristic of other pseudopotentials, as well. Examples include the PIMC simulation of Wallqvist et al., ${ }^{215}$ and the QCMD study of Barnett et al. ${ }^{203}$ Subsequent improvements of the pseudopotentials led to a more satisfactory agreement of the position of the spectral maximum with experiment. ${ }^{93,103}$ Inclusion of solvent electronic polarizability made it possible to fine tune the band maximum to near the exact position. ${ }^{103}$ The high energy tail of the spectrum, however, is still not fully developed in these models. We note here that Staib and Borgis was the first to apply the well-known Kubo-formula for computing the frequency resolved absorption spectrum of the hydrated electron (see Eq (9)). ${ }^{93} \mathrm{We}$ remind that the formula contains the time autocorrelation function of the dipole moment operator 
averaged over the electronic eigenstates and the solvent degrees of freedom. In a promising attempt, Gallicchio and Berne also used the time correlation function formula expressed in terms of a time-ordered displacement correlation function. ${ }^{86}$ The imaginary-time displacement correlation functions have been used to compute the hydrated electron absorption spectrum using PIMC simulation data with satisfying result. ${ }^{86}$ Nevertheless we note that the simulated spectrum underestimates the width, and also the variation in width with temperature is opposite to experiments.

The most recent work on this problem, due to Jacobson and Herbert, ${ }^{105}$ suggests that inclusion of self-consistent treatment of solvent polarizability in a carefully benchmarked potential may be sufficient to remedy the notorious problems in the shape of the absorption band. $^{22,116,128}$ In fact, although the computed absorption band improved significantly, and this is probably the best available spectral shape computed with one-electron pseudopotentials, the spectral shape is still not perfect. Notably, the red-edge of the spectrum has a mismatch, while the high-energy tail above $\sim 3 \mathrm{eV}$ is still significantly underestimated (Figure 10). ${ }^{128}$ Unlike all the pseudopotentials listed here that predict cavity arrangement for the hydrated electron, the recent electron-water pseudopotential developed by Larsen et al. yields a non-cavity structure. ${ }^{19}$ The Larsen potential predicts a spectrum red-shifted by $0.2 \mathrm{eV}$ with a significant depletion of the oscillator strength above $2.5 \mathrm{eV} .{ }^{19}$ An interesting comparison of the spectral calculations was performed by Herbert and Jacobson ${ }^{116}$ using three different pseudopotential models, the one by Turi and Borgis, ${ }^{103}$ the Larsen-Glover-Schwartz ${ }^{19}$ potential and the Jacobson-Herbert pseudopotential. ${ }^{105}$ They performed time-dependent DFT (TDDFT) spectral calculations on selected configurations generated from molecular dynamics using each of the three examined potentials. ${ }^{116}$ The calculations using the Turi-Borgis potential basically reproduce the experimental spectrum, while those using the Jacobson-Herbert configurations shift the spectral maximum with $0.3 \mathrm{eV}$ to the blue, and those with the Larsen-Glover- 
Schwartz set redshift the data by $0.5 \mathrm{eV} .{ }^{116}$ Based on these results, we conclude that the problem of reproducing the experimental spectrum of the ground state hydrated electron using pseudopotential methods remains incompletely resolved.

The second main source of discrepancy between experiment and theory is the neglect of the effect of the quantum behavior of the solvent bath on the calculated spectra. The Kubo formula, as we discussed in Section 3, opens a possibility to an a posteriori quantization of the calculated absorption spectrum. ${ }^{181}$ The quantized spectrum can be written in the following form:

$I^{q}(\omega)=\frac{\omega}{2 \pi}(1-\exp (-\beta \hbar \omega)) \times \sum_{k=1} \int_{-\infty}^{\infty} d t \exp \left(-i\left(\omega-\left\langle\Omega_{k 0}\right\rangle\right) t\right) \times \exp \left\{-\int_{0}^{t} d \tau_{1} \int_{0}^{\tau_{1}} d \tau_{2} C_{\Omega_{k 0}}^{q}\left(\tau_{2}\right)\right\} \times C_{\mu_{k 0}}^{q}(t)$

Eq. (11) contains $C_{\Omega_{k 0}}^{q}$, the quantized energy gap autocorrelation function, where the energy gap is calculated for the $0 \rightarrow k$ transition as $\Omega_{k 0}(t)=\left(E_{k}(t)-E_{0}(t)\right) / \hbar$, and $C_{\mu_{k 0}}^{q}$, the transition dipole moment autocorrelation function. The classical form of the autocorrelation functions are evaluated from QCMD simulations and are quantized using the harmonic quantization procedure. $^{216,217}$ It was found that the quantization procedure noticeably influences the shape of the absorption spectrum, especially its high-energy tail that arises from transitions to delocalized electron states. The inclusion of nuclear quantum effects improves the agreement between theory and experiment for both the low and high frequency edges of the spectrum. Thus, it seems likely that simultaneously addressing the two sources of errors is required for a satisfactory reproduction of the optical absorption spectrum in the oneelectron framework.

We note that most models that propose alternatives to the cavity picture are based on arguments related to the absorption spectrum. In particular, Tuttle and Golden compared optical absorption spectra of solvated electrons to those of $\mathrm{F}$ centers in alkali-metal halides 
and to those of solvated iodide ions. ${ }^{183}$ They suggest the solvated solvent-anion complex as the carrier of the hydrated electron spectroscopic and chemical properties. ${ }^{183}$ The hydrated hydronium radical complex advocated by Sobolewski and Domcke ${ }^{184}$ has spectroscopic properties that are consistent with the observed bulk infrared and electronic spectra. TDDFT/B3LYP calculated vertical electronic excitation energies of $\mathrm{H}_{3} \mathrm{O}\left(\mathrm{H}_{2} \mathrm{O}\right)_{3 \mathrm{~m}}, m=0,1,2$ clusters, convoluted with a Gaussian function of $0.7 \mathrm{eV}$ half-width, are surprisingly similar to the bulk hydrated electron spectrum. A crude ab initio based resonance Raman simulation by Neumann et $a l^{218}$ on similar species qualitatively reproduces the characteristic bands of Tauber and Mathies. ${ }^{57}$

We note that the two ab initio molecular dynamics studies of which we are aware on the bulk hydrated electron resulted in spectral characteristics that resemble to experiment (Figure 10), ${ }^{131,132}$ although limited statistics clearly hinder a comparison to qualitative features. As a technical point, Boero observed that the self-interaction error correction in DFT-based CPMD simulations hardly influences the shape of the absorption spectrum. ${ }^{132} \mathrm{We}$ note here that it has been more recently demonstrated by Shkrob et al. ${ }^{127}$ and by Herbert and Jacobson $^{116}$ that DFT excitation energies of the hydrated electron do appear at the correct places.

The electronic absorption spectra of size selected hydrated electron clusters were measured by Ayotte and Johnson in the $n=6-50$ size range. $^{47}$ The spectra strongly blueshift and broaden with increasing cluster size (Figure 11). Although the position of the maximum quickly moves toward the maximum of the bulk species, it reaches only $\sim 1 \mathrm{eV}$ at $n=50$. The non-trivial size dependence of the spectra ${ }^{47}$ reheated the debate regarding the existence of surface vs interior state clusters. Systematic simulations of the size dependence of hydrated electron clusters were performed in the one-electron simulation framework. ${ }^{14,190,203}$ The earlier simulation of Barnett et al. predicted that the spectra of surface state clusters are 
located at considerably lower energies than the interior state spectra. ${ }^{203}$ Turi, Rossky and coworkers confirmed this behavior for a different pseudopotential model (Figure 11). ${ }^{14,190}$ The observed size dependent peak position in the Ayotte-Johnson experiment ${ }^{47}$ is mimicked in the simulations of surface state spectra, gradually shifting to the blue as the clusters grow. ${ }^{14,190}$ Such effect is not observed for interior states (Figure 11). In fact, the interior state spectra do not vary much with size being located basically at the simulated bulk spectral position, $\sim 2$ $\mathrm{eV}{ }^{14,190}$ The progression of surface states to the infinite size limit leads to surface hydrated electrons at the infinite water/air interface. Extrapolation of small cluster data yields a peak at $1.7 \mathrm{eV}$ at infinite size at $200 \mathrm{~K},{ }^{190}$ somewhat larger than the value $(1.5 \mathrm{eV})$ found from interface simulations, ${ }^{212}$ but in any case red of the peak position for the bulk. ${ }^{103}$ For comparison, the simulated absorption spectrum of the bulk hydrated electron using the same pseudopotential appears with a maximum at $1.9 \mathrm{eV} .{ }^{103}$ Spectral calculations for hydrated electron clusters are not yet available with two other recent pseudopotential models (Jacobson-Herbert ${ }^{105}$ and Larsen-Glover-Schwartz ${ }^{19}$ models), and these will be helpful to further evaluate the capabilities of the one-electron models. Similarly, absorption spectra for hydrated electron clusters from many-electron AIMD techniques would serve as potentially important benchmarks. 


\section{On other dynamical aspects of the equilibrium hydrated electron}

The hydrated electron is a prototype particle in many respects. The simplicity of the solute, a single electron in a water bath makes it an ideal probe to study the microscopic details of solvation dynamics. Equilibrium properties of the hydrated electron are strongly influenced by solvent fluctuations at ambient conditions, as we already discussed for equilibrium energetic, structural, and spectroscopic properties. In fact, the coupling between the solute and the solvent can be related to certain dynamical phenomena via the fluctuationdissipation theory, and we overview such dynamics in this section. First, we inspect solvation dynamics via linear response theory then extend the investigated properties to the diffusion coefficient of the hydrated electron. The observable signature of the presence of the excess electron upon the solvent matrix will be examined by looking at the simulated infrared spectrum of the hydrated electron. At the end of Section 6, we also review studies that simulate hydrated electron properties under different thermodynamic conditions.

\subsection{Solvation dynamics and equilibrium energy fluctuations}

Equilibrium simulations provide a convenient and relatively simple tool to study nonequilibrium phenomena in electron hydration. In electron solvation dynamics, the solvation response following an instantaneous perturbation of an equilibrium system is usually characterized by a non-equilibrium response function, $S(t),{ }^{219}$

$$
S(t)=\frac{\langle U(t)\rangle_{n e}-\langle U(\infty)\rangle_{n e}}{\langle U(0)\rangle_{n e}-\langle U(\infty)\rangle_{n e}}
$$

where $U(t)$ corresponds to the solvation energy at time $t$, and the "ne" subscript shows averages over an ensemble of non-equilibrium trajectories. In the linear response (small perturbation) limit, non-equilibrium perturbations of a system relax to equilibrium in the same 
way as spontaneous equilibrium fluctuations. The non-equilibrium $S(t)$ then coincides with the equilibrium time correlation function, $C(t)$, of the fluctuations of the solvation energy $(\delta U(t)=U(t)-\langle U\rangle),{ }^{220,221}$

$$
\frac{\langle\delta U(0) \delta U(t)\rangle}{\left\langle\delta U^{2}\right\rangle}=C(t)
$$

In the context of electron hydration, one can investigate two interesting scenarios. ${ }^{103}$ The relaxation of a ground state solvated electron can be characterized by the equilibrium response function of the ground state energy $\left(U(t)=E_{0}(t)\right)$, while the relaxation of an excited state electron, e.g., following excitation from the equilibrium ground state, may be approximated by the autocorrelation function of the quantum energy gap fluctuation $\left(U(t)=E_{1}(t)-E_{0}(t)\right)$. These two processes are closely connected to experiments involving the thermalization of a photoinjected excess electron and to the photoexcitation of a ground state electron, respectively. The corresponding non-adiabatic simulations will be reviewed in the following section.

Several molecular dynamics studies examined and utilized the linear response approximation when studying electron hydration. ${ }^{219,222,223,103}$ The solvent fluctuations directly influence the electron energy levels, and clearly appear in the fluctuations of the electronic energies (Figure 3). Solvent fluctuations can be identified in the solvent's spectral density, and the degree of impact on the energies analyzed. It has been found that while all regions of the spectrum influence individual electron energy levels, high frequency motions are weakly evident in the energy gap spectral density due to parallel fluctuations in the individual energy curves. Only those solvent modes that are significantly displaced upon perturbation (excitation) appear in the fluctuations of the quantum energy gap. ${ }^{222}$ The most important solvent fluctuation components to modulate the energy gap are translational modes, although librational motions also participate to a lesser extent. ${ }^{219,222,223}$ Explicit calculations of the 
response functions by Rossky and coworkers demonstrated that the non-equilibrium response following electron hydration behaves linearly. ${ }^{219,222,223}$ They also recognized and rationalized the difference between the ground state equilibrium response function, associated with photoexcitation from the ground state, and the response function of an equilibrium excited state, associated with a radiationless transition to the ground state, a "weaker" form of linear response behavior. $^{219,222,223}$ The general characteristics of the response functions are, nevertheless, similar. There are two main components, a shortest timescale relaxation $(\sim 10-20$ fs) with an inertial (Gaussian) behavior followed by an exponential relaxation on the $\sim 200$ $300 \mathrm{fs}$ timescale. The relative weight of the inertial part is large, usually more than $50 \%$ of the total response. Slight differences may appear in the response functions depending on the applied pseudopotential models, but this does not affect the main conclusions on electron hydration dynamics. Instantaneous normal mode (INM) analysis ${ }^{224}$ of electron hydration provided further insight into electron hydration dynamics. ${ }^{225}$ Introduction of differential INM spectra sheds light upon the changes solvent molecules experience upon photoexcitation of the solute. Low frequency diffusive and rotational modes and fast librational motions are influenced most by electron excitation. The computed INM response functions produce good general linear response behavior. The INM analysis also reveals that translational and librational motions govern the solvent response at the early stages of the dynamics ( $\leq 50 \mathrm{fs}$ ), while it is librational motions that dominate in the first half of this period. This observation leads to a prediction of an isotope effect in the inertial part of the response, an $~ 40 \%$ increase in the Gaussian timescale in deuterated water. This study adjusts previous observations where the response functions for electron hydration were found only a bit slower in deuterated water. $^{222}$

Before closing this subsection, we mention that an interesting relationship exists between solvation dynamics and the quantum decoherence timescale, as a result of their 
common dependence on the differences in nuclear forces between different electronic states. In particular, the short time solvent response for a solute/solvent bath is directly proportional to the timescale of quantum coherence loss. ${ }^{226}$ This relationship allows the prediction of quantum decoherence timescale in the hydrated electron system from the solvent response functions.

\subsection{Hydrated electron diffusion}

Understanding transport properties of ions is of particular interest in chemical and biological contexts. The fact that the simplest ion, the hydrated electron, exhibits an unusually high mobility relative to its ionic counterparts has generated considerable debate. Although electron transport investigations have a long history both experimentally and theoretically, here we limit ourselves to the discussion of the most recent experiments and first-principles studies only.

Experimentally, the mobility and the diffusion of the excess electron were measured by conductometric techniques. ${ }^{27}$ This latter property was observed to show an Arrhenius-type behavior. The transport properties of the hydrated electron have also been studied by numerous quantum molecular dynamics simulations. ${ }^{227,228,229,230,93,231}$ These simulations are mixed quantum-classical simulations, so the quantum mechanical character of the migrating electron is fully taken into account. As other common features, the simulations are run in an adiabatic fashion, and the dynamics is analyzed semi-classically by following the center-ofmass motion of the electron. Since the electron is well-localized in water, this is a sound approximation. Excess electron migration dynamics were first simulated at room temperature simultaneously by the Nitzan group in water cluster anions, ${ }^{227}$ and by the Rossky group in bulk water. ${ }^{228}$ These two adiabatic QCMD simulation studies explained hydrated electron diffusion in terms of polaron-like dynamics with electron-induced cluster reorganization that 
accompanies electron migration. This type of transport dynamics was argued to be responsible for the excess electron migration from the surface of large cluster anions toward the center of the cluster, to an interior state in $\left(\mathrm{H}_{2} \mathrm{O}\right)_{256}^{-} \cdot{ }^{227}$ Other simulations also verified faster excess electron diffusion compared to that of halide ions. ${ }^{228}$ The simulations also found that nonlocal transitions, quantum mechanical tunneling and non-adiabatic hopping need not be included in the model to explain experimental migration observations. Instead, the instantaneous response of the electron to solvent collective dynamics determines the diffusion process with a quasi-Brownian motion. The computed diffusion constant for the electron $\left(3.3 \times 10^{-5} \mathrm{~cm}^{2} / \mathrm{s}\right)^{228}$ is similar to that computed for the self-diffusion coefficient of water but smaller than the experimental value $\left(4.9 \times 10^{-5} \mathrm{~cm}^{2} / \mathrm{s}\right) .{ }^{27}$ It was found that the solvent model and the electron-water interaction influence the diffusion coefficient significantly, leading to a surprisingly wide range of simulated $D$ values. Three rigid model simulations using different electron-water pseudopotential predicted similar diffusion coefficients. Using a rigid RWK2 water model Barnett et al. received a $D$ value $\left(3.7 \times 10^{-5} \mathrm{~cm}^{2} / \mathrm{s}\right)^{229}$ similar to that of Schnitker and Rossky with rigid SPC model, ${ }^{228}$ and that by Staib and Borgis with a polarizable, rigid TIP4P $\left(4 \times 10^{-5} \mathrm{~cm}^{2} / \mathrm{s}\right) .^{93}$ The use of a different (softer) electron-water pseudopotential ${ }^{103}$ with the flexible SPC model enhances diffusion $\left(D=6.0 \times 10^{-5} \mathrm{~cm}^{2} / \mathrm{s}\right){ }^{105}$ The flexible RWK2-M model, on the other hand, leads to a much smaller $D\left(1.9 \times 10^{-5} \mathrm{~cm}^{2} / \mathrm{s}\right){ }^{229}$ Of the most recent hydrated electron models the model of Jacobson and Herbert overestimates the diffusion coefficient by $50 \%\left(D=7.9 \times 10^{-5} \mathrm{~cm}^{2} / \mathrm{s}\right),{ }^{105}$ while that of the non-cavity preferring potential of Larsen et al. ${ }^{19}$ predicts a too small $D$ by a factor of $2 .{ }^{105}$

The most recent comprehensive study on hydrated electron diffusion was performed by Tay et al. $^{231}$ They found Brownian-type behavior with a diffusion constant that is significantly larger than that of the solvent. The dominant solvent mode that couples to the instantaneous response of the electron is the libration. The mechanism of the diffusion is 
rationalized in terms of a transfer diffusion model ${ }^{232}$ involving an exchange of an extramolecular electron between identical water molecules. A rate constant of $5.0 \mathrm{ps}^{-1}$ for this second-order process is reported at room temperature. In accordance with experimental findings Arrhenius-type temperature dependence is found for electron diffusion in the 298 $400 \mathrm{~K}$ temperature range. The activation energy is computed to be $8.9 \mathrm{~kJ} / \mathrm{mol}^{231}$

\subsection{Vibrational density of states of the hydrated electron}

The main features of the IR spectrum of the hydrated electron system can be approximated from simulations by the Fourier transform of the velocity autocorrelation function of the water molecules. Direct comparison is possible with available experimental data. Resonance Raman spectroscopy measurements observe significantly downshifted $\mathrm{HOH}$ bending (by $\sim 30 \mathrm{~cm}^{-1}$ ) and $\mathrm{OH}$ stretching vibrations (by $\sim 200 \mathrm{~cm}^{-1}$ ). ${ }^{57}$ The earliest simulation attempt to predict the hydrated electron vibrational spectrum reaches back to the QCMD study of the smallest hydrated electron system, water dimer anion. ${ }^{89}$ Comparison of the characteristic frequencies to those of a neutral dimer did not hint at any characteristic change. Study of larger clusters does reveal distinct tendencies. ${ }^{203}$ The vibrational density of states of an interior state $\left(\mathrm{H}_{2} \mathrm{O}\right)_{256}^{-}$cluster indicates a blue-shift of the O-H stretch by about $200 \mathrm{~cm}^{-1}$, and no significant change in the bending region, incompatible results with experiment, in addition to a characteristic red-shift in the librational regime (consistent with experiment), and a blue-shift in the spectral region associated with the translational motion. ${ }^{203}$ We note that this is the only vibrational analysis for a one-electron model in the literature. ${ }^{203}$ It has been argued based on quantum mechanical vibrational calculations following a local harmonic approximation $^{127}$ and natural bond orbital analysis ${ }^{202}$ that one-electron models are unable to fully capture the experimentally observed characteristic shifts due to the need to include charge penetration. In particular, static many-electron DFT frequency calculations on cluster 
anion configurations taken from QCMD simulations surrounded by point-charge represented bath molecules suggest correct tendencies in comparison with the frequencies of neutral clusters in the same anionic geometry. ${ }^{127}$ Further, an analysis has been performed by Park et al. who used a hybrid quantum-classical simulation technique, treating the valence electrons of six water molecules and the excess electron quantum mechanically, embedded in a classical solvent bath. ${ }^{129}$ For the hydrated electron system this study predicts $\sim 40 \mathrm{~cm}^{-1}$ and $\sim 100 \mathrm{~cm}^{-1}$ downshift for the bending and the $\mathrm{O}-\mathrm{H}$ stretching vibrations, respectively, in reasonable agreement with experiment. The most ambitious calculation, BOMD simulations by Frigato et al., computed the IR spectrum for a $n=32$ surface state water cluster anion. ${ }^{135}$ The study analyzed the $\mathrm{H}-\mathrm{O}-\mathrm{H}$ bending region and found a $\sim 100 \mathrm{~cm}^{-1}$ red-shift that indicates the presence of double acceptor (AA) hydrogen-bonding motif in the cluster, ${ }^{135}$ in agreement with experiments. ${ }^{198,199}$ These many-electron calculations thus support the idea that charge penetration is necessary to explain the shifts in the vibrational spectrum. Nevertheless, we note that the charge penetration is estimated to be less than $20 \%,{ }^{127}$ and it remains to be resolved if this, in our opinion, relatively small effect does indeed lead to the assumed general qualitative inconsistency of the one-electron models.

\subsection{Hydrated electron simulations at different thermodynamic state points}

The first simulation study that considered hydrated electron equilibrium properties at different temperatures was performed by Wallqvist et al. using PIMC simulation technique. ${ }^{215}$ They investigated hydrated electron structure and the absorption spectrum at $300 \mathrm{~K}$ and 373 K. The solvation structure at the higher temperature reflects more diffuse electron-hydrogen and electron-oxygen pair correlation functions. However, the calculated absorption spectrum did not manifest the experimentally observed red-shift of the spectra with increasing temperature. ${ }^{41}$ Another PIMC study that used the same model of Wallqvist et al., ${ }^{215}$ and 
combined the simulated data with maximum entropy analytic continuation method, properly predicted red-shifting spectra with increasing temperature. ${ }^{86} \mathrm{~A}$ more recent one-electron QCMD simulation study by Nicolas et al. reports a significantly improved picture. ${ }^{233}$ They studied a wide range of conditions varying pressure and temperature of the system from ambient to supercritical conditions. The simulations employed the rigid SPC water potential in combination with the Turi-Borgis pseudopotential. ${ }^{103}$ The theoretical results were evaluated in comparison with the pulse radiolysis experiments of $\mathrm{Wu}$ et $a .^{234}$ Although the water structure around the hydrated electron seems to completely disappear at $400{ }^{\circ} \mathrm{C}$ and 0.48 $\mathrm{g} / \mathrm{cm}^{3}$ density, and a few water molecules are able to penetrate as close as $\sim 0.5 \AA$ to the center-of-mass of the electron distribution, the cavity around the negative charge is still preserved. The simulated spectra computed at five different thermodynamic conditions are shown in Figure 12. The experimental red-shift of the absorption spectrum with increasing temperature is reproduced, although not quantitatively. Microscopic interpretation of the tendency is attributed primarily to a density rather than to a temperature effect. The nonmonotonic behavior of the band width of the spectra is also recovered. Similar results were also obtained from AIMD simulation using the Car-Parrinello framework. ${ }^{132}$ As a relatively new development, recent experiments by $\mathrm{Du}$ et al. suggests that although density is an important variable in the temperature dependence of the hydrated electron spectrum, temperature variation in itself also plays some role, a question that remains to be explained in simulations. $^{235}$ 


\section{Non-equilibrium hydrated electron dynamics and spectroscopy in bulk, in clusters and on water/air infinite interfaces}

A fully microsocopic understanding of the elementary physical events involved in electron solvation is a challenge. Most striking is the fact that electron solvation takes place extremely rapidly, well within $\sim 1$ ps. With the advance of ultrafast laser spectroscopies it has become possible to record the experimental signatures of these complex processes with femtosecond time resolution. The development of new theoretical methods and algorithms has, at the same time, contributed significantly to the consistent interpretation of the experimental data. The hydrated electron has been an ideal model for studying various basic aspects of condensed phase dynamics; the excess electron, has a single electronic degree of freedom, and its coupling to the solvent dynamics can be relatively straightforwardly examined. This is especially true in one-electron simulation approaches, where information on the coupling between solute and solvent nuclear degrees of freedom is easily and directly accessible.

The two main experimental electron hydration scenarios in bulk water ${ }^{38,39}$ can be straightforwardly mimicked by performing molecular dynamics simulations. In the first one, an electron is photodetached from a guest solute, and, an excess electron is thus injected into

an equilibrium solvent bath with consequent electronic and solvent relaxation. ${ }^{38,39}$ The simplest treatment of the ensuing dynamics is adiabatic, when one supposes that the excess electron occupies its ground state during the dynamics. In non-adiabatic simulations, one can follow electronic transitions of an initially excited state electron during the relaxation to the fully hydrated electron state. In the second major setup, an equilibrium hydrated electron is excited to one of its excited states (photoexcitation), and the electronic relaxation back to its ground state is followed via distinct non-adiabatic electron transition steps and concomitant 
solvent relaxation. ${ }^{39}$ In these two types of experiments, the dynamics samples completely different initial conditions, the equilibrium neat solvent in the first case, the equilibrated hydrated electron in the second. The study of these processes provides complementary information on the microscopic details of electron hydration dynamics. Experimental observations and theoretical predictions can be contrasted via a direct comparison of the simulated and experimental transient spectral traces. The comparison is facilitated by the direct computation of transient absorption signals by Rossky and his group ${ }^{236,237,238,239,240}$ and by Bratos et al., ${ }^{241,242,243,244}$ as we will describe below.

Non-equilibrium hydrated electron cluster simulations have so far been mainly limited to adiabatic ground state relaxation following electron attachment to neutral water clusters. Here the challenge is to identify the dominant localization mode of the electron (interior state vs. surface state) and the mechanistic details of the associated structural evolution. Simulation of photoexcitation of hydrated electron clusters has not been reported yet. Similarly, interfacial hydrated electron simulations are almost exclusively limited to adiabatic electron attachment studies on water/air interfaces. In the present section, we will discuss these nonequilibrium electron hydration dynamics scenarios.

\subsection{Molecular dynamics simulations of excess electron photoinjection in neat water}

In photoinjection experiments, the excess electron can be created by radiolysis of water using ionizing radiation, UV multiphoton ionization of water, or ionization of electron donors in aqueous medium employing 2 or 3 laser pulse sequences. ${ }^{38}$ The electron dynamics is monitored by appropriately chosen probe laser pulses. At least two timescales have been observed in these experiments, suggesting the presence of precursor species to the fully hydrated electron. ${ }^{33,34}$ For the timescales Migus et al. inferred $110 \mathrm{fs}$ and $240 \mathrm{fs},{ }^{33}$ while Long et al. reported $180 \mathrm{fs}$ and $540 \mathrm{fs}^{34}$ The most extensive studies of this type, by Barbara and 
coworkers, first pointed out that the timescale for equilibration strongly depends on how the excess electron is prepared initially. Their observed timescales were $280 \mathrm{fs}$ and $400 \mathrm{fs} .{ }^{39}$ These experiments were interpreted to involve relatively high-energy, delocalized species that becomes localized in partially relaxed electronic states before the full relaxation to the ground state, with the concomitant heat release and solvent reorganization. The basic model that evolved from the observation of the spectral dynamics is the so-called two-state model: $33,34,245$

$$
\mathrm{e}_{\text {free }}^{-} \stackrel{k_{1}}{\longrightarrow} \mathrm{e}_{*}^{-} \stackrel{k_{2}}{\longrightarrow} \mathrm{e}_{\mathrm{s}}^{-}
$$

Here, $\mathrm{e}_{\mathrm{free}}^{-}$means the delocalized "free" or "quasifree" electron, $\mathrm{e}_{*}^{-}$is a localized species, sometimes called the "wet" or "presolvated" electron, and $\mathrm{e}_{\mathrm{s}}^{-}$is the equilibrium hydrated electron.

The equilibrium neat solvent provides well defined initial conditions for simulating photoinjection experiments. Since the initial conditions of the energy and size distribution of localization sites are of primary importance in the initial steps of solvation, an analysis of these features provides important information on the early stages of the dynamics. After an electron is injected in an equilibrium water bath, the electron solvation begins with the localization of the initially delocalized excess charge. Schnitker at al. analyzed water configurations that were generated by classical molecular dynamics using a test charge. ${ }^{246}$ They found a relatively high number density of favorable sites both in terms of size and electrostatic potential. The picture suggests that short-range interactions support the initial energetic stability. The quantum mechanical approach analyzing the ground state excess electronic energy for selected water configurations results in similar conclusions, ${ }^{247,248}$ indicating that classical calculations can be used as a qualitative indicator for the electron localization ability of the solvent. The calculations show that although the classical potential energy estimator is negative for all the cases, the electron's ground state energy is always 
positive. Similar analysis performed with another water-electron pseudopotential model ${ }^{103}$ led to the same qualitative conclusions but with $0.4 \mathrm{eV}$ deeper excess electron stabilization energy and a more delocalized electron distribution. ${ }^{126}$

The primary events on the shortest timescale $(<1 \mathrm{fs})$ following electron injection into bulk water and water layers were investigated by Barnett et al. ${ }^{249}$ They directly solved the time dependent Schrödinger equation and found that the electron momentum relaxes very rapidly after the creation of the electron, within $\sim 0.5$ fs. Nevertheless, it was observed that the energy transfer to water is relatively slow and insignificant on the femtosecond timescale. At the earliest stages of the dynamics water molecules do not play a significant role in the electronic relaxation, and the process may be modeled by an interaction of the electron with a collection of static water molecules. ${ }^{249}$

The subsequent steps of the relaxation including localization were first simulated by Rossky and Schnitker using adiabatic one-electron QCMD technique, where they restricted the electron to occupy its ground state from the onset of dynamics, ${ }^{88}$ neglecting the participation of electronic relaxation. Simulations, in parallel with experiment, predicted two timescales, $\sim 30 \mathrm{fs}$ and $\sim 200 \mathrm{fs}$, associated with a fast localization and the following heat dissipation and translational reordering. Nevertheless, it was evident that the neglect of excited excess electron states and the associated non-adiabatic electronic transitions, predicted too fast dynamics. ${ }^{88}$

Non-adiabatic effects in electron hydration dynamics have been introduced in two different ways, a) using the Fermi golden rule formula to extract transition rates from adiabatic simulations and b) by direct non-adiabatic molecular dynamics simulations. Of the two methods, the first one is more applicable to the photoexcitation of the hydrated electron, which we review in the next subsection. The direct non-adiabatic technique developed by Webster et al. ${ }^{92}$ employs a surface hopping method in combination with the stationary phase 
approximation based non-adiabatic dynamics forces of Pechukas. ${ }^{159}$ This NA technique was first used by Webster et al. ${ }^{250}$ followed by additional studies ${ }^{251,252}$ to explicitly treat nonadiabatic transitions following electron photoionization in neat water. A typical representation of such a NA trajectory can be seen in Figure 13, illustrating non-radiative transition events between excess electron states. This early work recognized two major classes of relaxation trajectories. ${ }^{250}$ The first channel is associated with a rapid cascade of NA steps through the excited states directly to the ground state. Once the electron reached the ground state (50-150 fs range) it is rapidly solvated. Alternatively, on the same timescale, the electron was seen to form a well-defined solvated excited state, and then make a transition to the ground state within approximately 1 ps. The calculated transient spectrum $^{250}$ reflecting hydration dynamics mimicked experiment, although it evolved more quickly in comparison with experiment. ${ }^{33,34}$ An isosbestic point was found in the simulation in agreement with Long et al. ${ }^{34}$ providing support for the two state solvation model. The remaining discrepancies between experiment and theory were partly accounted for in subsequent works using flexible water models in MD simulations. $^{251,252}$ Not surprisingly, the relaxation was found to take place more rapidly ${ }^{251,252}$ than with rigid water models. ${ }^{250}$ The direct relaxation to ground state occurs in $\sim 25 \mathrm{fs}$, while the excited state lifetime was $\sim 160$ fs, comparable to some experiments. ${ }^{33,34}$ Nevertheless, it was argued that the characteristic lifetime should fall between the values found with the rigid model and those with the model that employs classical intramolecular vibrations. ${ }^{251}$ A kinetic analysis of the trajectories described the relaxation mechanism ${ }^{252,253}$ with a series of fast thermalization steps across the manifold of excited states, followed by competition between a direct trapping channel to the electron ground state and a two-step path involving a welldefined excited hydrated electron state.

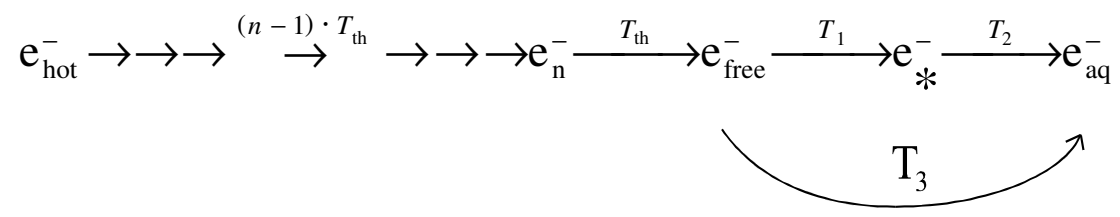


Here $\mathrm{e}_{\mathrm{hot}}^{-}$denotes hot electrons with excess energy precluding them from localization, and $T_{\text {th }}$ is the typical lifetime of state in dense manifold of electronically hot states with $\mathrm{e}_{\mathrm{n}}^{-}$ being the $n^{\text {th }}$ state in this manifold. All the other species are identical to that in Eq. (14). Nevertheless, we note that the physical identity of the experimentally observed events could not be certain in this photoinjection case. For this reason a better defined experiment was sought, as discussed next.

\subsection{Molecular dynamics simulations of photoexcitation experiments of an equilibrium}

\section{hydrated electron}

A conceptually different type of experiment were devised and performed in the Barbara group in the early 90's. ${ }^{35,254,255,256}$ They performed near IR pump-probe transient absorption spectroscopy of the fully equilibrated, hydrated electron. The electron is promoted by a pump pulse to a low lying excited state, and the subsequent dynamics is monitored by a probe pulse. The simplified scheme of the basic relaxation events is shown in Figure 14. The mechanism involves non-adiabatic steps between the p-state and the s-state and among the pstates, in addition to electronically adiabatic components of solvation dynamics for both the sstate and the p-state electron. Since the conceptual rationalization of photoexcitation experiments is simpler than that for photoinjection, this type of experiment has become a favorite target for both experimentalists and theoreticians. Using polarized pump and probe pulses (polarized transient hole-burning) has further widened the applicability of the technique providing additional insight into the anisotropy of hydrated electron dynamics and spectroscopy. ${ }^{257}$ Similar experiments followed from Assel et al., ${ }^{258,259,260}$ more recent measurements by Barbara and his coworkers, ${ }^{38,39}$ Pshenichnikov et al. ${ }^{36}$ and Thaller et al. ${ }^{261}$ The measured decay times, however, cover a remarkable range and can be grouped into three categories. The earliest work of the Barbara group ${ }^{254,255}$ and Assel et al. ${ }^{258,260}$ predict $\sim 200 \mathrm{fs}$ 
for the NA decay time of the excited state electron followed by an $\sim 1$ ps ground state solvent relaxation. Isotope effects were not observed with the $300 \mathrm{fs}$ available time resolution in deuterated water. ${ }^{255}$ More recent work of Barbara and his co-workers ${ }^{35,256}$ proposed a $\sim 35-80$ fs timescale regime that exhibits a solvent isotope effect $\left(\tau\left(\mathrm{D}_{2} \mathrm{O}\right) / \tau\left(\mathrm{H}_{2} \mathrm{O}\right)=1.4\right)$ and is attributed to inertial/librational motion of the solvent, a $\sim 300$ fs excited p-state adiabatic lifetime followed by a slower NA decay with $\sim 1$ ps time constant. These models constitute the socalled 'adiabatic' solvation model. Pshenichnikov et al. performed photon-echo experiments with very short ( $\sim 5 \mathrm{fs})$ laser pulses and inferred an extremely short, $\sim 50$ fs excited state lifetime. ${ }^{36}$ Photo-imaging pump-probe measurements of Verlet et al. on small water cluster anions extrapolated to infinite cluster size were interpreted with a very similar p-state lifetime. ${ }^{13}$ This very different interpretation of the photoexcitation setup, a very rapid NA transition followed by two slower timescale decays of ground-state relaxation is the so-called 'non-adiabatic' solvation model. Different type of experiments, however, which probe the presence of the excited state electron more directly via selective scavangers ${ }^{16,38,39,262}$ are inconsistent with such a fast NA decay placing the lifetime in the much longer 300-500 fs range. With such diversity regarding timescales, theoretical methods can provide useful guidelines as to which interpretation is most likely to be correct.

That the situation is incompletely resolved is evidenced by the fact that available theoretical results are also scattered in a relatively wide range. QCMD-based results strongly depend on the applied interaction model and the quantum simulation method. The first such approach was introduced by Neria et al. ${ }^{174,175}$ who combined a standard quantum mechanical expression for the transition rate (Fermi golden rule) with the application of Gaussian wavepackets for the nuclear modes as a high-temperature quantum correction. Due to the assumptions of the model, this approach estimates the NA transition rate (lifetime) of an equilibrated excited state electron. Neria and Nitzan applied the method to an interior state 
water cluster anion containing 128 molecules. ${ }^{174,175}$ Their estimated lifetimes for the cluster excited state electron are $\sim 220 \mathrm{fs}$ in $\left(\mathrm{H}_{2} \mathrm{O}\right)_{128}^{-}$and $\sim 800 \mathrm{fs}$ in $\left(\mathrm{D}_{2} \mathrm{O}\right)_{128}^{-} \cdot{ }^{175}$

A similar route was followed by Staib and Borgis who also used the golden rule expression, ${ }^{93}$ with an a posteriori nuclear quantum effect correction of the correlation functions appearing in the golden rule formulation. The quantization is performed to make the correlation function symmetric in time and obey the detailed balance condition. The analysis then employs linear response arguments, and predicts $\sim 230$ fs $(\sim 500$ fs without nuclear quantization) for the equilibrated lifetime.

In the most recent approach, Borgis and co-workers also employed a quantum time correlation function formula for the NA decay rate between two adiabatic quantum states based on the Fermi golden rule. ${ }^{177}$ The formula was applied to estimate the lifetime of the equilibrium excited state hydrated electron in bulk water. This work also used an a posteriori quantization procedure to take nuclear quantum effects into account, similar in spirit to the original work of Staib and Borgis. ${ }^{93}$ The computed lifetime of the equilibrium excited state hydrated electron obtained was extremely short, under 100 fs. ${ }^{177}$ The apparent discrepancy with experiment was identified as associated with the strong dependence of the rate on the energy gap between the excited and ground states, that in turn, depends on the degree of solvation of the excited state solute. ${ }^{263}$ Solvation dynamics thus plays a critical role in the observable decay. An energy gap-dependent transition rate can be formulated using linear response theory and implemented in a kinetic equation for the survival probability function of the excess electron excited state. The analysis including solvation dynamics provides an apparent lifetime for the excited state hydrated electron of $\sim 300 \mathrm{fs},{ }^{263}$ in accord with a preponderance of the experimental results. ${ }^{16,38,39,262}$

A series of direct NA molecular dynamics studies simulating photoexcitation experiments have also appeared. Schwartz and Rossky performed direct NA QCMD 
simulations, ${ }^{219}$ starting from an equilibrated ground state electron configuration. A typical simulated trajectory is shown in Figure 15. The average lifetime of the trajectories, $\sim 700 \mathrm{fs}$, provides an estimate for the average p-state lifetime. ${ }^{219}$ Comparison with photoinjection simulations with the same model ${ }^{250}$ showed that the excited state residence time following photoexcitation is roughly 5 times longer than for electrons trapped in a p-state after photoinjection, in qualitative agreement with experiment. A statistical analysis of the trajectories also allows construction of a survival probability function giving the occupation probability of the excited state as a function of time. ${ }^{219}$ The solvent response correlation functions were characterized by a 25 fs Gaussian inertial component and a 250 fs exponential decay $^{219}$ correlating well with experiment ${ }^{256}$ and with those found by Barnett et al. in finite clusters for the excited state hydrated electron using a different flexible classical model and a pseudopotential (see below). ${ }^{204}$ The detailed relationships between solvation dynamics and the experimentally determined emission Stokes-shift were explored in other study. ${ }^{240}$ The molecular level analysis of the solvent mode participation in the non-radiative relaxation was also performed showing that solvent librations and the water asymmetric stretching mode are observed to be the most effective promoters of the non-adiabatic transitions. ${ }^{264}$

Although simulated transient photophysical hole-burning spectroscopy of the hydrated electron was first addressed via adiabatic molecular dynamics simulations, ${ }^{236}$ full account of all important contributions, ground state bleaching, excited state absorption and stimulated emission, was carried out and analyzed by Schwartz and Rossky, ${ }^{237,238}$ by calculating spectra on both adiabatic ground and excited state trajectories in parallel. The computed spectral transients (shown in time and frequency domains in Figure 16) show remarkable agreement with experiment. ${ }^{254,255}$ Both the direct NA simulations with the kinetic analysis ${ }^{219}$ and the simulated hole burning transient spectra ${ }^{237,238}$ suggest dominance of excited state solvation dynamics followed by a relatively slow radiationless NA transition to the ground state, with a 
subsequent, very fast ground state cooling. This picture was further supported by a statistical theory using correlation function description of the non-linear optical processes of nonpolarized and polarized ultrafast transient absorption spectroscopy developed by Bratos and Leickman. ${ }^{241,242,243,244}$

Initial simulations of the equilibrium hydrated electron photoexcitation in $\mathrm{D}_{2} \mathrm{O}$ resulted in a large solvent isotope effect on the characteristic lifetimes, increasing by about a factor of two, predicting $\sim 1.5 \mathrm{ps}$ for the average decay time and $\sim 850 \mathrm{fs}$ for the equilibrium lifetime of the excited state hydrated electron in neat $\mathrm{D}_{2} \mathrm{O} .^{222}$ This was a puzzling result because experiments performed with 300 fs time resolution laser pulses found no significant differences between the spectral evolution in water and in deuterated water. ${ }^{255}$ The simulated solvent responses in $\mathrm{H}_{2} \mathrm{O}$ and $\mathrm{D}_{2} \mathrm{O}$ were found to be similar, but later INM analysis showed $\sim 40 \%$ increase in deuterated water ${ }^{225}$ in good agreement with the experimental ratio of 1.4 using significantly better time resolution. ${ }^{256}$ A plausible explanation for the large difference in lifetimes in NA simulations was traced to a need for the correct treatment of quantum decoherence times in light and heavy water. ${ }^{265}$ Once decoherence times were correctly estimated and implemented in simulations (2.7 fs in $\mathrm{H}_{2} \mathrm{O}$ and 4.0 fs in $\mathrm{D}_{2} \mathrm{O}$ ), the lifetimes became comparable in the two solvents. As a general finding, it was observed that correction for decoherence leads to significantly faster NA transition with an excited state lifetime of $\sim 200$ fs. This trend is also expected to hold for the computed rates found in photoinjection simulations. Another factor, nuclear quantum effects, can also significantly change the excited state lifetime in QCMD simulations. Simulations have estimated that quantum treatment of the vibrations further increases the rate by about $30-50 \%{ }^{176,265}$

Larsen et al. further analyzed the methodological issues by systematically performing photoexcitation simulations for an equilibrium ground state electron using various oneelectron NA QCMD techniques. ${ }^{153}$ They found that all analyzed NA methods estimate 
qualitatively the same average lifetime ranging 400 - 700 fs. Similarly, the non-equilibrium solvent response functions are nearly identical in all examined QCMD simulation techniques. The most recent attempt to rationalize the experimental findings has also been done by Larsen et al. as a demonstration of the capabilities of their newly introduced non-cavity forming electron-water pseudopotential. ${ }^{19}$ The computed transient hole-burning spectral traces agree well with the experimental signals. ${ }^{255}$ The microscopic dynamical picture of this model predicts that the excited state relaxation takes place very rapidly followed by a transition to the ground state in $\sim 280 \mathrm{fs}$, and a $\sim 1 \mathrm{ps}$ timescale slower ground state re-equilibration. This interpretation is consistent with the later experiments of Barbara and his colleagues. ${ }^{256}$ Hence, we conclude that these experiments do not distinguish among the different physical pictures arising from different models.

Of the most recent attempts to estimate the excited state solvated electron lifetime, a different approach was adapted by Zharikov and Fischer. ${ }^{67}$ Their continuum hydrated electron model (using scaling theory) provides an analytical estimate for the non-radiative lifetime of the excited state electron that is less than $100 \mathrm{fs}$, significantly shorter than any calculations using explicit molecular models. Nevertheless, this result appears to be in qualitative agreement with Pshenichnikov et al. ${ }^{36}$ and the cluster studies of Verlet et al. $^{13}$

Simulations of ultrafast polarization-dependent spectral hole-burning experiments further illustrate the difficulties encountered in the attempts to fully resolve electron hydration phenomenon. The first such simulation by Schwarz and Rossky predicted significant experimentally detectable differences in the timescales of the isotropic and anisotropic solvent fluctuations. ${ }^{238}$ This finding was reflected in a clear, persistent simulated spectral signature that the first polarized hole-burning experiments seemed to confirm. ${ }^{257}$ These results, however, were later challenged by both theoretical ${ }^{242,266,19}$ and experimental studies. ${ }^{36,258,259,267}$ We mention here Shkrob's analysis that points to a possible source of the 
discrepancies in the application of soft (less repulsive) versus hard (more repulsive) pseudopotentials in the one-electron simulations. ${ }^{266}$ Larsen et al. reached a similar conclusion using their non-cavity preferring pseudopotential, ${ }^{19}$ but Herbert and Jacobson have reported that the lack of polarized hole burning dynamics is not necessarily inconsistent with the cavity model itself. ${ }^{105}$

In summary, both experimental and theoretical results cover a considerable range for the lifetime of the excited state hydrated electron (Table 2). One may notice that all the firstprinciple based molecular dynamics methods (regardless of the applied classical model or pseudopotential) estimate several hundred fs for the average lifetime. These numbers are in good general qualitative agreement with the most recent experimental lifetime estimates of 300-500 fs, based on the application of specific scavangers. ${ }^{16,38,39,262}$ We emphasize that the reported bulk water photon-echo results ${ }^{36}$ and anionic water cluster lifetime measurements ${ }^{13}$ that suggest ultrashort ( $<100 \mathrm{fs})$ excited state lifetimes in bulk water and very large clusters remain to be reconciled with the great majority of other experiments that provide excited state lifetimes in the several hundred fs range. The most readily interpreted experiments based on hot electron scavengers fall into the latter group. ${ }^{16,262}$

7.3. Non-equilibrium molecular dynamics simulations of excess electrons in water clusters and on water/air interfaces

The number of experimental studies on hydrated electron cluster dynamics is significantly more limited than for the bulk hydrated species. An obvious reason for this is that experiments on cluster anions are even more technically demanding, with the cluster anion preparation and selection adding a layer of complexity. Despite the challenges, the debate surrounding the details of bulk electron hydration has motivated the extension of timeresolved measurements to hydrated electron clusters. Time-resolved photoelectron 
spectroscopy experiments have been performed on finite size hydrated electron systems. The earliest such measurement on $\left(\mathrm{H}_{2} \mathrm{O}\right)_{n}^{-}$clusters with $n=20-100$ developed an upper bound for the lifetime of the excited state comparable to the order of the pulse width, $150 \mathrm{fs}^{48}$ Paik et al. observed two timescales in time-resolved PES experiments on water cluster anions with $n=15-35,300$ fs and 2-10 ps, both attributed to ground state solvent relaxation following NA transition. ${ }^{11}$ The Neumark group performed a remarkable and comprehensive series of timeresolved photo-imaging PES experiments on $\left(\mathrm{H}_{2} \mathrm{O}\right)_{n}^{-}$and $\left(\mathrm{D}_{2} \mathrm{O}\right)_{n}^{-}$in the $n=25-200$ size range. ${ }^{13,195,209,210,211}$ The experiments reveal that the excited state lifetimes are cluster sizedependent (decreasing with increasing cluster size), depend on the electron binding motif of the cluster, and manifest large solvent isotope effects. Type I clusters extrapolate at large size to an excited state lifetime of $\sim 60 \mathrm{fs}$, while type II appears to extrapolate to $\sim 250 \mathrm{fs} .\left(\mathrm{D}_{2} \mathrm{O}\right)_{n}^{-}$ clusters relax more slowly by a factor of 2 . The experimental results for type I and II clusters are shown in Figure 17.

Although there are no direct experimental data on the dynamics of hydrated electron cluster formation via electron attachment, theoretical studies of this process can suggest relevant microscopic details of cluster dynamics and offer insights into the process. A first step of such an analysis is the examination of potential electron localization sites of neutral water clusters. Localization site analysis for finite size hydrated electron systems at different temperatures has been performed using one-electron models. ${ }^{126}$ The study indicates that initially excess electrons localize preferentially on pre-existing localization sites on neutral cluster surfaces and on water/air interfaces. Even for relatively small simulated clusters $(n \sim 30$ and larger), most of the neutral clusters support bound localized states on the surface, and as size increases, almost all neutral cluster configurations have negative electron binding energy. The initial binding energy increases with size. The stabilization of the electron is strongly correlated with the preexisting instantaneous dipole moment of the neutral clusters. Favorable 
binding sites within the clusters were not found. These findings suggest that electron attachment to neutral clusters is consistent with an initial surface state. ${ }^{126}$ Similar conclusions were also drawn much earlier by Landman et al. based on one-electron PIMD simulations on small hydrated electron clusters. ${ }^{82}$

Water cluster anion dynamics were first explicitly examined by Barnett et al. ${ }^{204}$ They performed adiabatic QCMD simulations following instantaneous electronic transitions from equilibrated ground state to the first electronic excited state and from equilibrated excited state to the ground state of the excess electron in $\left(\mathrm{H}_{2} \mathrm{O}\right)_{64}^{-}$and $\left(\mathrm{H}_{2} \mathrm{O}\right)_{128}^{-}$clusters that bind the excess electron in an interior state. They observed a 20-30 fs fast inertial component, and a slower 250 fs decay for the electronic state energies, energy gaps, and electronic radii following both types of instantaneous transitions. These timescales do not appear to be sensitive to the size of the clusters. These simulations agree with the later NA simulation results of Schwartz and Rossky for bulk hydrated electrons. ${ }^{219}$

Interestingly, while, the adiabatic non-equilibrium dynamics of surface state clusters have not been examined with one-electron simulation methods, recently Jungwirth and coworkers have extended the AIMD methodology to the water cluster anion problem, performing both equilibrium and relaxation studies on finite-size hydrated electron clusters. ${ }^{135,136,137,138,139}$ A Born-Oppenheimer molecular dynamics run on a warm $(T=350 \mathrm{~K})$ $\left(\mathrm{H}_{2} \mathrm{O}\right)_{32}^{-}$cluster where the excess electron was prepared in an initially localized interior state indicated that the electron remains stable in the cavity for about $0.5 \mathrm{ps}$, then evolves reaching a stable localized surface state at $\sim 3$ ps after the start of the simulation. The surface state structure remains relatively stable for the remaining length of the 15 ps long trajectory with apparent surface delocalization - localization fluctuation events. ${ }^{135}$ A subsequent BOMD study revealed the molecular details of the electron attachment process to neutral clusters. ${ }^{138}$ Excess electrons were attached in their ground state to equilibrated neutral clusters of 
different temperature and the subsequent temporal evolution of the systems was followed and monitored. At ambient temperature $(300 \mathrm{~K})$ the initially delocalized electron (of $\sim 6 \AA$ radius) undergoes a sizable reduction in size within the first $1 \mathrm{ps}$, while the surface solvent molecules reorient mainly by local rotational motions. Further translations and rotations create a polarized "dent" on the surface where the electron is further stabilized, reaching its final radius of $2.75 \AA$ in less than $1.5 \mathrm{ps}$, while always remaining at the interfacial region of the cluster. $^{138}$ The situation dramatically changes in cold clusters $(20 \mathrm{~K}$ and $50 \mathrm{~K})$. While the initial localization timescale of the events appears similar to the $300 \mathrm{~K}$ case, further relaxation is very different. The excess electron does not localize further, its radius remains high $(\sim 4 \AA)$, and its VDE remains relatively small. Apparently, the excess electrons are trapped, presumably kinetically, in these, at least metastable, states where they can survive for a relatively long period on the timescale of this simulation $(\sim 5 \mathrm{ps}) .{ }^{138}$ Similar conclusions that question the validity of extrapolations to ambient bulk water hydrated electron properties from cold clusters were reached by molecular dynamics simulations of cold clusters. ${ }^{137}$ Here, in addition to examining electron surface localization on quenched neutral clusters of 32 water molecules, the authors simulated the behavior of a quenched $\left(\mathrm{H}_{2} \mathrm{O}\right)_{32}^{-}$water cluster anion that was previously equilibrated at $300 \mathrm{~K}$. The observed physical properties appear dramatically different in the two examined scenarios for the same cluster size when prepared by distinctly different procedures at low temperatures $(<200 \mathrm{~K}) .{ }^{137}$

Non-adiabatic simulations on water cluster anions were performed by Neria et al. simulating non-adiabatic transition rates of excited state equilibrium electrons to the ground state, ${ }^{174,175}$ as already noted, for $\left(\mathrm{H}_{2} \mathrm{O}\right)_{128}^{-}$and $\left(\mathrm{D}_{2} \mathrm{O}\right)_{128}^{-}$clusters. The lifetime of the equilibrated excited state electron (see Table 2) for $\mathrm{H}_{2} \mathrm{O}$ is reasonable in comparison with experiment, ${ }^{13}$ but the $\mathrm{D}_{2} \mathrm{O}$ lifetime seems to be significantly overestimated. The linear dependence of the excited state hydrated electron cluster lifetime on the inverse of the cluster 
radius was also rationalized by Fischer and Dietz in terms of a very simple, one-electron quantum model. ${ }^{268}$ The size dependence was attributed to a non-adiabatic long-range coupling mechanism between the $\mathrm{p} \rightarrow \mathrm{s}$ electronic transition and the excitation of the IR active modes of the water molecules. ${ }^{268}$

Experimental preparation and detection of hydrated electrons on infinite liquid water surfaces have been successfully carried out quite recently. The dynamical details suggest long-lived species on the surface, with a ground state lifetime of $\sim 100$ ps. ${ }^{9,18}$ Even longer lived species (up to minutes) were detected on ice surfaces absorbed on metal substrates after electron injection into the ice conduction band. ${ }^{269}$ In particular, time- and angle-resolved twophoton-photoemission indicates timescales ranging from fs to ps in amorphous ice layers on $\mathrm{Cu}(111)$, while for amorphous and crystalline $\mathrm{D}_{2} \mathrm{O} / \mathrm{Ru}(001)$ the stabilization energy further increases and the timescales extend up to minutes. ${ }^{50,270,271,272}$ The processes that are anticipated to play a fundamental role in the electron dynamics at the interfaces are electron injection, localization, solvation and simultaneous back transfer to the metal. The simulation of this complex scheme is beyond the capabilities of the computational techniques that have been implemented so far. Nevertheless, simpler models can capture the physics of certain aspects of the complex mechanism. Adiabatic molecular dynamics simulations of the electron localization, surface solvation and surface state to interior state transition have been performed in a one-electron scheme at ambient water/air, supercooled water/air, Ih ice/air and amorphous solid water/air interfaces. ${ }^{212}$ The simulations suggest that initially the electron localizes in a shallow potential trap on the interface. The initial localization occurs on the $\sim 20$ fs timescale, while further relaxation takes place through a sequence of distinct surface state structures that are distinguishable by the energetics, geometries and hydrogen-bonding patterns. ${ }^{212}$ In the case of ambient water the excess electron slowly (on the $\sim 10$ ps timescale) 
diffuses into the bulk, while on colder surfaces the electron remains (again presumably kinetically) trapped at the interface during the timeframe of the simulations. 


\section{Related topics}

Here we briefly discuss some questions closely related to the hydrated electron. It is not our intent to be complete in this coverage but to point out some topics that have been identified in the same theoretical literature. These topics include that of the role of the hydrated electron in the dynamics of charge-transfer-to-solvent (CTTS) excitations of halide and related ions and the molecular dynamics simulations of hydrated electron reactivity. We also summarize the most recent findings on the dynamics and reactivity of the hydrated dielectron in bulk water and finite size clusters.

\subsection{Hydrated electrons in the dynamics of CTTS states of halide ions}

Aqueous solutions of halide anions possess broad absorption bands in the UV associated with the displacement of the highest energy bound halide electron to an excited state supported by solvent polarization. The electronic transition leads to the formation of so called charge-transfer-to-solvent states and ultimately to the hydrated electron. ${ }^{273}$ The photoexcitation of the CTTS bands has been proved to be an efficient way to prepare hydrated electrons. ${ }^{274}$ Several time-resolved experiments have been performed to investigate excited CTTS state dynamics and monitor the subsequent hydrated electron dynamics. ${ }^{275,276,277,278,279}$ The cluster analogs of bulk CTTS transitions and the ensuing dynamics have also been observed and studied by the Johnson group ${ }^{280}$ and the Neumark group. ${ }^{281,282}$ The latest experimental development in the field involves the application of picosecond and femtosecond transient X-ray absorption spectroscopy to probe the structure and the dynamics of aqueous halide systems. ${ }^{283}$

The CTTS dynamics was studied extensively in the one-electron QCMD picture by Sheu and Rossky ${ }^{284,285,286,287}$ and by Staib and Borgis. ${ }^{93,288}$ Sheu and Rossky analyzed the 
CTTS spectra of aqueous halide ions, ${ }^{284}$ the electronic dynamics of photoexcited iodide in a water bath, ${ }^{285}$ and the dynamics of the electron detachment from an aqueous halide ion. ${ }^{286}$ This latter work monitored the dynamics using non-adiabatic simulation techniques. Two channels were identified in halide ion relaxation dynamics following initial excitation to the lowest halide CTTS state. The minor channel is a direct photodetachment, with the excited plike electron making a non-adiabatic transition directly to a well-separated hydrated electron and a parent halogen atom. The dominant channel, which was first identified from these simulations, takes place via relaxation through the manifold of CTTS states followed by an adiabatic detachment process to the ground state of the hydrated electron. ${ }^{286}$ The relaxation dynamics was further analyzed in a subsequent study. ${ }^{287}$ It was found that branching between the two channels occurs at very early times, within the first 50 fs of the dynamics. The timescale of the subsequent electron detachment steps, however differ by an order of magnitude, the direct channel being significantly faster than the adiabatic detachment channel. The role of solvent dynamics has been emphasized as playing a critical role in determining the rate of electron separation and also that of geminate recombination by electron transfer onto the halogen atom. ${ }^{287}$

Staib and Borgis employed equilibrium and non-equilibrium adiabatic QCMD simulations to study the photodetachment of an electron from aqueous chloride ions. ${ }^{93,288}$ In particular, they focused on the relaxation dynamics from the lowest $4 s$ CTTS state. On this route, a predominant channel was also identified that leads to a metastable hydrated electronchlorine atom contact pair. Starting from the contact pair two pathways were identified, a dissociation channel yielding hydrated electron and a geminate recombination path to chloride ion via a non-adiabatic transition. For this latter channel the self-consistent treatment of solvent electronic polarizability was found to play an important role. ${ }^{288}$ 
To the best of our knowledge, many electron AIMD simulations have not been performed yet on the transition of the CTTS state to hydrated electron. However, small solvent cluster simulations of the CTTS states of halide ions have been reported, ${ }^{283,289}$ providing the first step toward the complete mechanistic simulation.

\subsection{Ab initio molecular dynamics studies of hydrated electron reactivity}

The hydrated electron is a ubiquitous highly reactive species that plays a major role in radiation-induced chemistry. Understanding hydrated electron reactivity has both significant practical and theoretical implications. ${ }^{8}$ An important example that has been investigated for decades is the hydrated electron quenching reaction with hydronium cations to form hydrogen gas. Although this is a seemingly simple, elementary reaction in aqueous radiation chemistry, its importance stems from major concerns in nuclear waste reprocessing and storage. ${ }^{8}$

One electron QCMD techniques using classical water models are not applicable to such problems. Jungwirth and his colleagues were the first to simulate proton transfer to a hydrated electron using ab initio BOMD/DFT techniques with large diffuse basis sets and self-interaction corrections. ${ }^{136,139}$ The reaction has been modeled in a water cluster containing 31 water molecules, one hydronium cation and an excess electron. Ten trajectories of several ps length were launched starting from selected configurations of a corresponding long classical (non-reactive) simulation. The trajectories showed that while the electron is initially localized in a solvent cavity and diffuses relatively slowly, the proton moves more rapidly by a site-hopping mechanism. ${ }^{290}$ Only three of the ten trajectories resulted in reaction, to atomic hydrogen, within the timeframe of the simulations, but these indicate that the predominant mechanism involves the electron as a proton acceptor. It is found that for the reaction to take place, it is necessary for one or two water molecules to penetrate relatively deeply into the excess electron density to become more reactive. This penetration is accompanied by a 
distortion of the excess electron from its symmetrical shape to a highly prolate distribution. Then the reactive event takes place with one proton passed to the electron, the electron density collapsing around that proton and the remaining hydroxyl ion accepting another proton from its hydrogen-bonding network to restore the water molecule. Although not a statistically established result, the simulated sample indicates that the reaction is slower than diffusion-limited; the quenching reaction was observed after many proton hops along the hydrogen-bonded chain with several unsuccessful encounters with the electron. The main reason for a reaction barrier appears to be the desolvation penalty for the charged particles during the association reaction and the hydrophobicity of the product hydrogen atom. ${ }^{136,139}$ This argument is consistent with experimental observations that the reaction is not diffusionlimited, ${ }^{291}$ taking place about 5 times slower than diffusion limited at room temperature. ${ }^{292}$

CPMD methods have also been applied to study chemical reactions involving hydrated electron formation. Most notably Renault et al. examined the hydrogen atom - hydroxide anion reaction that produces hydrated electrons using DFT/BLYP approximation. ${ }^{293}$ Simulations pointed to a complex mechanism that is dominated by proton transfer in the coordination sphere of the hydroxide ion and the diffusion of the hydrogen atom in its solvent cavity. The mechanistic details of hydrated electron formation indicate that water antibonding orbitals play a key role in the hydration of the electron.

\subsection{The dynamics and reactivity of the hydrated dielectron}

After the original discovery of the hydrated electron, the possibility that two excess electrons can spin pair in a water bath forming a hydrated dielectron was considered. Motivated by early experiments, ${ }^{294}$ Fueki proposed the first continuum dielectric model for the hydrated dielectron. ${ }^{295}$ Subsequent experiments have not led to complete agreement on the 
existence of the hydrated dielectron. ${ }^{296,297}$ Nevertheless, the hydrated dielectron has been invoked as an intermediate in the bimolecular water reduction reaction. ${ }^{291,296,298}$

Theoretical studies on the hydrated dielectron are relatively few. Early continuum ${ }^{295}$ and semi-continuum models ${ }^{299}$ predicted stability of the hydrated dielectron relative to two separate hydrated electrons. The first extensive quantum molecular dynamics study came from the Landman group. ${ }^{300}$ Here two electrons are treated quantum mechanically in $\left(\mathrm{H}_{2} \mathrm{O}\right)_{n}^{2-}$ clusters of classical solvent molecules with $n=64,128,256$. The electron-bath interaction is described by a pseudopotential, ${ }^{102}$ while the ground-state electronic structure of the two electrons is computed in the local-spin-density approach, ${ }^{301}$ and the nuclear dynamics is propagated on the Born-Oppenheimer ground state surface. It is found that the dielectron in a spin-paired state is stable only in the largest water cluster examined, $n=256$. The ground state dielectron adiabatic binding energy is estimated to be in the range $-4--7 \mathrm{eV}$. The stability of the dielectron is mainly attributed to the long-range interaction of the electrons with the water molecules of the surrounding medium with a significant contribution from exchange, as well. Further energetic parameters (the adiabatic dissociation energy of the dielectron and the vertical ionization energy) were also computed. ${ }^{301}$ Structurally, the dielectron is predicted to occupy a solvent-supported state confined to a single cavity in the solvent. The shape of the cavity is observed to fluctuate rapidly between a state of roughly spherical symmetry and an elongated, ellipsoidal, dumbbell-shaped cavity, with approximately degenerate electronic states in these configurations. The radius of the dielectron state in the spherical configuration is only a bit larger than the radius of the hydrated electron. $^{301}$

More than 10 years later, the Schwartz group published a series of papers on hydrated dielectrons. ${ }^{302,303,304,305}$ After the cluster studies of Kaukonen et al., ${ }^{300}$ the Schwartz group simulated bulk hydrated dielectron properties using a two-electron mixed quantum/classical 
simulation technique. Adiabatic simulations using the Schnitker-Rossky pseudopotential model $^{88}$ with full configuration interaction for the eigenstates also predicted the formation of dielectrons. ${ }^{302}$ The two excess electrons form a pair in a singlet or a triplet fashion in a solvent cavity with $-6.0 \mathrm{eV}$ and $-4.7 \mathrm{eV}$ ground state energies, respectively. The size of the cavity appears to be larger than for the hydrated electron. The solvent structure around the excess charges exhibits $\mathrm{OH}$ bond directed orientation with a first-shell coordination number of $\sim 9$. Spectroscopic characterization of the simulated hydrated dielectron has also been performed. The optical absorption spectra of the dielectron species appear to the blue of the hydrated electron suggesting that experiments should focus on the blue-tail of the spectrum for the identification of the dielectron. ${ }^{302}$ Although the singlet-state dielectron state appears to be stable with respect to dissociation in MD simulations, thermodynamic integration indicated that the dielectron is thermodynamically unstable. ${ }^{303}$ Kinetic stability may, however, be sufficient to allow experimental observation. Simulations suggest scenarios for creating nonequilibrium dielectrons via the capture of a newly injected excess electron by a preexisting hydrated electron.

Subsequent non-adiabatic simulations evaluated the dynamics ${ }^{304}$ and the pump-probe spectroscopy ${ }^{305}$ of the hydrated dielectron following photoexcitation. The spin-singlet and spin-triplet dielectrons are found to relax on different timescales. While singlet state dieletrons relax to the ground state on timescales similar to the hydrated electron, spin-triplet dielectrons relax much faster, and the difference has been explained in terms of exchange and correlation effects. ${ }^{304}$ Simulations of transient spectroscopy indicate clear pump-probe signatures that can be used to distinguish between singlet-state hydrated dielectrons and hydrated electrons. $^{305}$

The first experimental observation of the hydrated dielectron has been reported in water clusters recently by Barnett et al. ${ }^{140}$ Water cluster anions were produced by supersonic 
expansion of water vapor in Ne carrier gas intersected by an electron beam. The time-of-flight mass spectrometer indicated three types of clusters: singly-charged water cluster anions dominate the spectrum, but smaller peaks also appear in the $83 \leq n<105$ range suggesting the presence of doubly charged water cluster anions, $\left(\mathrm{H}_{2} \mathrm{O}\right)_{n}^{2-}$. At higher masses, peaks shifted by two mass units due to hydrogen loss appear as well. Ab initio Born-Oppenheimer molecular dynamics simulations were presented in the same report, using DFT with the PBE exchangecorrelation functional. ${ }^{143}$ For singlet-paired dielectrons, two stable isomeric cluster geometries are identified for the hydrated dielectron, as shown in Figure 18. In the most energetically stable bonding motif, the two excess electrons are both localized on the surface of the cluster, on opposite sides, so as to minimize electrostatic repulsion. The VDE's are small compared to the results of two-electron model-based MD simulations, ${ }^{300,302,303}$ but still stabilizing; the energy of the doubly charged cluster anion has been computed at $1.3 \mathrm{eV}$ below that of the neutral cluster. The configuration where two electrons occupy an interior cavity is only $0.3 \mathrm{eV}$ more stable than the neutral cluster configuration, with a relatively large cavity radius of $\sim 5.9$ $\AA$ A. This interior dielectron state would correspond to the bulk hydrated dielectron in the infinite cluster size limit. Temporal evolution of the dielectron system exhibits large fluctuations, and, it is concluded that, at finite temperatures, the observed ensembles of doubly-charged clusters would likely be comprised of a variety of excess electron localization modes. ${ }^{140}$

Barnett et al. also attempted to locate the reaction pathway of dielectron hydrogen evolution. ${ }^{140}$ Since experimentally the hydrogen evolution is observed for $n \geq 105$, they performed steered BOMD simulations on the hydrogen evolution reaction for the $n=105$ cluster:

$$
2 \mathrm{H}_{2} \mathrm{O}+2 \mathrm{e}^{-} \rightarrow 2 \mathrm{OH}^{-}+\mathrm{H}_{2}
$$


The reaction started from an interior dielectron cluster is found to take place via a concerted approach of two protons from two first shell water molecules. The individual steps in the mechanism appear to be highly cooperative and very rapid, basically completed on a subpicosecond timescale. For smaller clusters, the reaction leading to hydrogen is not observed, a result attributed to the lack of stability of the interior state dielectron. ${ }^{140}$ 


\section{Summary and Outlook}

In this review, we have summarized a remarkably large number of studies focused on what might have been imagined to be a very simple system, simpler than the simplest atom dissolved in water. In considering properties ranging from the solvation structure and the optical spectrum of the hydrated electron to the dynamics of electron detachment from excited state anions, we hope it has become clear that this simplicity is deceptive. While the preponderance of the models used are consistent with a cavity picture in which the excess electron density is self-trapped - localized by the solvent, organized by the electronic charge itself - and primarily contained in a solvent-free void within the water, even this most basic aspect of the structure is still debated among serious researchers in the field. One can conclude that the value of the electronic excited state lifetime in ambient bulk water appears to be around $500 \mathrm{fs}$, but this value is not universally accepted and the striking cluster size dependence in anionic clusters points to an important gap in completing our picture. The relative importance of the excited state lifetime and solvation dynamics on the observed transient spectroscopy is likewise still in open discussion. The detailed investigation of anionic water clusters via both theory and experiment has begun to yield considerable new insights into the hydrated electron, as well as revealing the additional structural and dynamical scenarios possible in cold size-selected clusters but absent in the bulk liquid. In particular, the presence of both surface- and interior-localized electronic states, and the apparent dependence of these states on cluster "temperature" and the method of cluster preparation, has generated a very rich field for investigation that has assumed a central position in research studies, quite separate from its potential role in elucidating the bulk hydrated electron species. 
The numerous research examples reviewed should also have made clear that research in the area has consistently evidenced a high level of intellectual integration of theory and experiment in the gradual refinement of our understanding of the underlying microscopic structure and dynamics. Since the hydrated electron species is the simplest hydrated electronic system that can exist, it has lent itself to theoretical attack from the inception of studies of the species. Nevertheless, we are confident that the reader can appreciate the remarkable growth in theoretical methods and models which has taken place over the past about 40 years of studies, so that now ab initio methods of dynamics are emerging as the frontier approach. For example, theory has been essential to the use of spectroscopy, providing a framework within which one can distinguish and identify distinct structural forms of anionic molecular clusters. Dynamical simulations have provided the window on processes needed to determine the elementary molecular events which are reflected in ultrafast spectroscopy of electronic excited states. Further, theory has been at the heart of understanding the mechanism of photoinduced electron detachment, and promises to quantitatively describe corresponding excited state processes in molecular systems in the future. Excited state dynamics is an area where there remains considerable limitations on such $a b$ initio methods, and one must anticipate considerable additional growth in that aspect.

Despite the effort that has been expended in studies of the hydrated electron and closely related systems, the outlook for new studies remains quite bright. As noted, there remains substantial debate surrounding the most basic elements of structure and excited state dynamics, and so there are real opportunities for investigations that are less dependent on simplified models than the studies now in the literature. In the area of anionic water clusters, in particular, the detailed connection between the cluster preparation step and the cluster structure is still generally unclear, and the structural commonalities in studied clusters that lead to an apparently relatively small number of cluster structural motifs are still puzzling. 
Further, the relationship between structure and electronic dynamics is not yet well developed. Farther into the future, the reactions of hydrated electrons will be a rich area, having only been touched on at this point in time, despite the fact that interest in the species has always emanated primarily from the high reactivity of the species in energetically excited materials. Study of reactive mechanisms for solvated electrons should provide a proving ground for methods focusing on the ultrafast dynamical steps of chemical reaction in solution, and in condensed soft matter, more generally. We therefore have no doubt that there will be good reason to write another chapter on theoretical methods applied to hydrated electrons in the not too distant future. 


\section{Acknowledgments}

The work was supported by a grant to L. T. from the National Research Fund of Hungary (OTKA) under Contracts No. K75285. P. J. R. is grateful to the National Science Foundation (CHE-0910499) and the R. A. Welch Foundation (F-0019) for support of this work. 
Table 1. Selected geometrical parameters of the bulk hydrated electron computed from computer simulations.

\begin{tabular}{|c|c|c|c|c|c|c|c|}
\hline Authors & Reference & Method & Structure & $\begin{array}{c}\text { Electron radius } \\
(\AA)\end{array}$ & $\begin{array}{l}\text { Electron-hydrogen } \\
\text { rdf maximum }(\AA)\end{array}$ & Water orientation & $\begin{array}{c}\text { Coordination } \\
\text { number }\end{array}$ \\
\hline \multicolumn{8}{|c|}{ one-electron models } \\
\hline Wallqvist et al. & 85. & PIMC & cavity & $2.11,2.24$ & - & bond & 4 \\
\hline Schnitker et al. & 79,88 & PIMD/QCMD & cavity & 2.4 & 2.3 & bond & 6 \\
\hline Turi et al. & 103. & QCMD & cavity & 2.42 & 2.1 & bond & 4 \\
\hline Jacobson et al. & 105. & QCMD & cavity & 2.25 & 1.7 & bond & 4 \\
\hline Larsen et. al & 19. & QCMD & non-cavity & $2.46^{2} / 2.69^{3}$ & 0.8 & - & - \\
\hline \multicolumn{8}{|c|}{ many-electron models } \\
\hline
\end{tabular}

${ }^{2}$ Ref. 19 using minimum image simulations.

${ }^{3}$ Ref. 116 using Ewald summation to correct for the long-range interactions.

${ }^{4}$ Measured in an aqueous glass at $T=77 \mathrm{~K}$. 
Table 2. Calculated excited state hydrated electron lifetimes

\begin{tabular}{|c|c|c|c|c|c|}
\hline Author & Ref. & Method & $\tau_{\text {equilibrium }}{ }^{5}$ & $\tau_{\text {apparent }}{ }^{6}$ & remark \\
\hline \multirow[t]{2}{*}{ Nitzan et al. } & 174,175 & Golden rule, QCMD, Gaussian wavepackets & $220 \mathrm{fs}$ & & $\left(\mathrm{H}_{2} \mathrm{O}\right)_{128}^{-}$ \\
\hline & & & $800 \mathrm{fs}$ & & $\left(\mathrm{D}_{2} \mathrm{O}\right)_{128}^{-}$ \\
\hline Staib et al. & 93 & Golden rule, QCMD, correlation fct. quantization & $230 \mathrm{fs}$ & $200-300 \mathrm{fs}$ & \\
\hline Borgis et al. & 177 & Golden rule, QCMD, correlation fct. quantization & $<<100$ fs & $330 \mathrm{fs}$ & \\
\hline \multirow[t]{3}{*}{ Schwartz et al. } & 219 & SPSH & $450 \mathrm{fs}$ & $730 \mathrm{fs}$ & \\
\hline & 222 & & $850 \mathrm{fs}$ & $1.5 \mathrm{ps}$ & $\mathrm{D}_{2} \mathrm{O}$ \\
\hline & 265 & SPSH with corrected decoherence timescale & & $200 \mathrm{fs}$ & $\mathrm{H}_{2} \mathrm{O} / \mathrm{D}_{2} \mathrm{O}$ \\
\hline \multirow[t]{3}{*}{ Larsen et al. } & 153 & MF-SD & & $630 \mathrm{fs}$ & \\
\hline & 153 & MFSH & & $450 \mathrm{fs}$ & \\
\hline & 153 & Tully's method & & $410 \mathrm{fs}$ & \\
\hline Larsen et al. & 19 & Tully's method + non-cavity pseudopotential & & $280 \mathrm{fs}$ & \\
\hline Zharikov et al. & 67 & Continuum hydrated electron model & & $<100 \mathrm{fs}$ & \\
\hline
\end{tabular}

\footnotetext{
${ }^{5}$ Equilibrium lifetime is the lifetime of the excited state in an equilibrated excited state hydrated electron system.

${ }^{6}$ The apparent lifetime is the average lifetime of the excited state electron.
} 


\section{Figure Captions}

Figure 1. The experimental optical absorption spectrum of the bulk hydrated electron at $T=$ $300 \mathrm{~K}^{40}$

Figure 2. Excess electron densities in the potential of a water molecule and a repulsive confining potential. Values given along the dipole direction of a water molecule in the molecular plane through the oxygen atom. The center of mass of the water molecule is at the origin, the hydrogen atoms are at negative coordinates. The exact pseudopotential theory result for the density (black) is compared to the densities implied by two pseudopotential models: Larsen, Glover and Schwartz (blue) ${ }^{19}$ and Turi and Borgis (red). ${ }^{103}$ The corresponding electron-water potentials are shown in the bottom panel.

Figure 3. Typical time evolution of the ground state and the first five excited state energies of a hydrated electron as obtained from one-electron quantum molecular dynamics simulations. ${ }^{190}$

Figure 4. Electron-hydrogen (red) and electron-oxygen (blue) radial distribution functions between the center of mass of the electron and the atomic sites of the water molecules as obtained from one-electron QCMD simulations using a model pseudopotential that yields a cavity state $^{103}$ and a non-cavity state. ${ }^{19}$

Figure 5. Excess electron densities in a bulk water bath as obtained from one-electron QCMD simulations using a model pseudopotential that yields a cavity state ${ }^{103}$ and a non-cavity state. ${ }^{19}$ The isosurfaces contain $80 \%$ of the electron distribution.

Figure 6. The relationship between interior state and surface state bound water cluster anions, and their infinite size counterparts, the bulk hydrated electron and the infinite surface hydrated electron. The isosurfaces contain $80 \%$ of the electron distribution. 
Figure 7. Radius of gyration $r_{\mathrm{e}}$ and kinetic energy $E_{\mathrm{kin}}$ of the excess electron at $200 \mathrm{~K}$ for surface state cluster anions (squares) and interior state cluster anions (triangles). The insets show a part of the data on an expanded scale. Empty and full symbols denote simulations in Refs. 14 and 190, respectively. Reprinted with permission from Ref. 190 (Madarász, Á.; Rossky, P. J.; Turi, L. J. Chem. Phys. 2009, 130, 124319). Copyright 2009 American Institute of Physics.

Figure 8. Experimental (top panel) and simulated (bottom panel) VDE values of water cluster anions as a function of cluster size. Top panel: Squares represent the experimental data of Ma et al. (Ia: red, Ib: blue, Ic: green). ${ }^{196}$ The experimental data of Verlet et al. are shown (I: open circles, II: open triangles, III: open squares). ${ }^{13}$ Bottom panel: The AIMD simulated values of Barnett et al. are shown with green (interior state: square, surface state: triangle, diffuse surface state: circle). ${ }^{140}$ The simulated data of Jacobson and Herbert using a one-electron model are shown with red (interior state: square, partially embedded surface clusters: triangle, proper surface isomers: circle). ${ }^{206}$ The simulated data of Turi et al. are shown with black (interior state: square, surface state: triangle). ${ }^{14,190}$ Open symbols are the energies calculated from a dielectric continuum theory (interior state: square, surface state: triangle). ${ }^{66,190}$

Figure 9. Correlation between VDE of the excess electron and its radius in $\left(\mathrm{H}_{2} \mathrm{O}\right)_{32}^{-}$in different simulation setups, following electron attachment to cold water clusters (blue), following electron attachment to ambient water clusters (green), following simulations of cold clusters with preformed interior states (cyan), and equilibrium anionic simulations at ambient $T$ (red). Reprinted with permission from Ref. 139. Copyright 2012 American Chemical Society.

Figure 10. Simulated and experimental optical absorption spectra of the bulk hydrated electron. The experimental spectra are shown by dashed line. Top panel: the absorption spectrum computed using a one-electron PIMD simulation (continuous line). ${ }^{87}$ The dotted 
curve represent the experimental curve shifted to the blue by $0.7 \mathrm{eV}$. Center panel: the absorption spectrum computed by Jacobson and Herbert using one-electron QCMD simulation (continuous line). ${ }^{105}$ Bottom panel: the optical spectrum computed by Boero et al. using CPMD technique (continuous line). ${ }^{131,132}$

Figure 11. Simulated and experimental electronic absorption spectra of size selected hydrated electron clusters. Top panel: photodestruction data of Ayotte and Johnson. ${ }^{47}$ Center panel: simulated surface state clusters for $n=45-8000$ (45: blue, 200: green, 1000: red, 8000: black). Bottom panel: interior state clusters for $n=200-8000$ (200: blue, 500: green, 1000: red, 8000: black).

Figure 12. Optical absorption spectra at five different thermodynamic conditions following the experiments of $\mathrm{Wu}$ et $a .^{234}$ : circles $\left(25^{\circ} \mathrm{C}, 1.0 \mathrm{~g} / \mathrm{cm}^{3}\right)$, squares $\left(100{ }^{\circ} \mathrm{C}, 0.96 \mathrm{~g} / \mathrm{cm}^{3}\right)$, diamonds $\left(250{ }^{\circ} \mathrm{C}, 0.82 \mathrm{~g} / \mathrm{cm}^{3}\right)$, triangles $\left(350{ }^{\circ} \mathrm{C}, 0.63 \mathrm{~g} / \mathrm{cm}^{3}\right)$, starts $\left(400{ }^{\circ} \mathrm{C}, 0.48 \mathrm{~g} / \mathrm{cm}^{3}\right)$. Reprinted with permission from Ref. 233 (Nicolas, C.; Boutin, A.; Lévy, B.; Borgis, D. J. Chem. Phys. 2003, 118, 9689). Copyright 2003 American Institute of Physics.

Figure 13. A typical non-adiabatic trajectory showing the time evolution of the occupied electronic state (solid curve) and other unoccupied (dashed curves) excess electron states in the simulation of a photoinjection experiment. The excess electron has an initial excess energy of $\sim 2 \mathrm{eV}$. Reprinted with permission from Ref. 252 (Keszei, E.; Nagy, S.; Murphrey, T. H.; Rossky, P. J. J. Chem. Phys. 1993, 99, 2004). Copyright 2004 American Institute of Physics.

Figure 14. A simplified scheme describing the excitation and non-radiative relaxation mechanism of the hydrated electron photoexcited to a p-like state.

Figure 15. A typical non-adiabatic trajectory showing the time evolution of the occupied electronic state (solid curve) and other unoccupied (dashed curves) excess electron states in 
the simulation of a photoexcitation experiment. The excess electron is initiated in a p-state at a configuration that is resonant with a designated pump pulse energy ( $t=0 \mathrm{fs}$ ). Reprinted with permission from Ref. 219 (Schwartz, B. J.; Rossky, P. J. J. Chem. Phys. 1994, 101, 6902). Copyright 2004, American Institute of Physics.

Figure 16. Simulated transient spectral hole burning traces for the hydrated electron. a) Simulated time domain spectral transients for the hydrated electron at various wavelengths. b) Frequency domain traces at various time delays. Reprinted with permission from Ref. 237. Copyright 1994 American Chemical Society.

Figure 17. Experimental lifetimes of an excited state hydrated electron in various size $\left(\mathrm{H}_{2} \mathrm{O}\right)_{n}^{-}$and $\left(\mathrm{D}_{2} \mathrm{O}\right)_{n}^{-}$clusters. Reprinted with permission from Ref. 211. Copyright 2009 American Chemical Society.

Figure 18. Two stable modes of attachment of two excess electrons in $\left(\mathrm{H}_{2} \mathrm{O}\right)_{105}^{2-}$ clusters. (A) shows a surface localization modes, while (B) is an interior attachment mode. Reprinted with permission from Ref. 140. Copyright 2011 American Chemical Society. 
Figure 1. Turi and Rossky

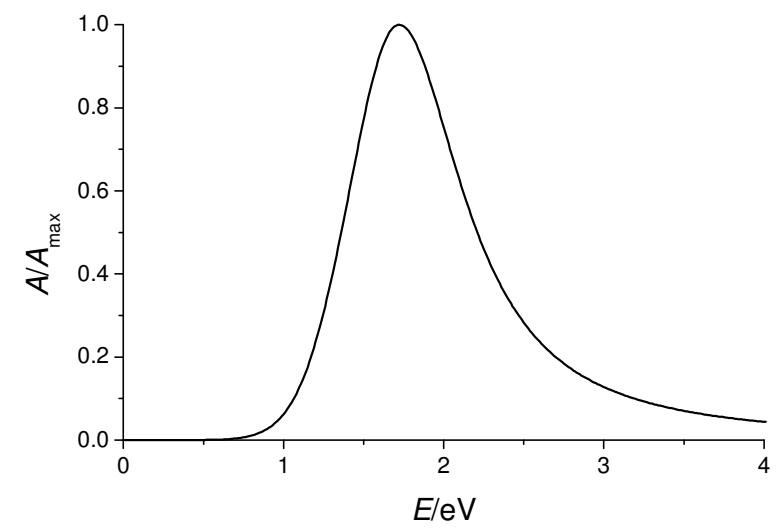


Figure 2. Turi and Rossky

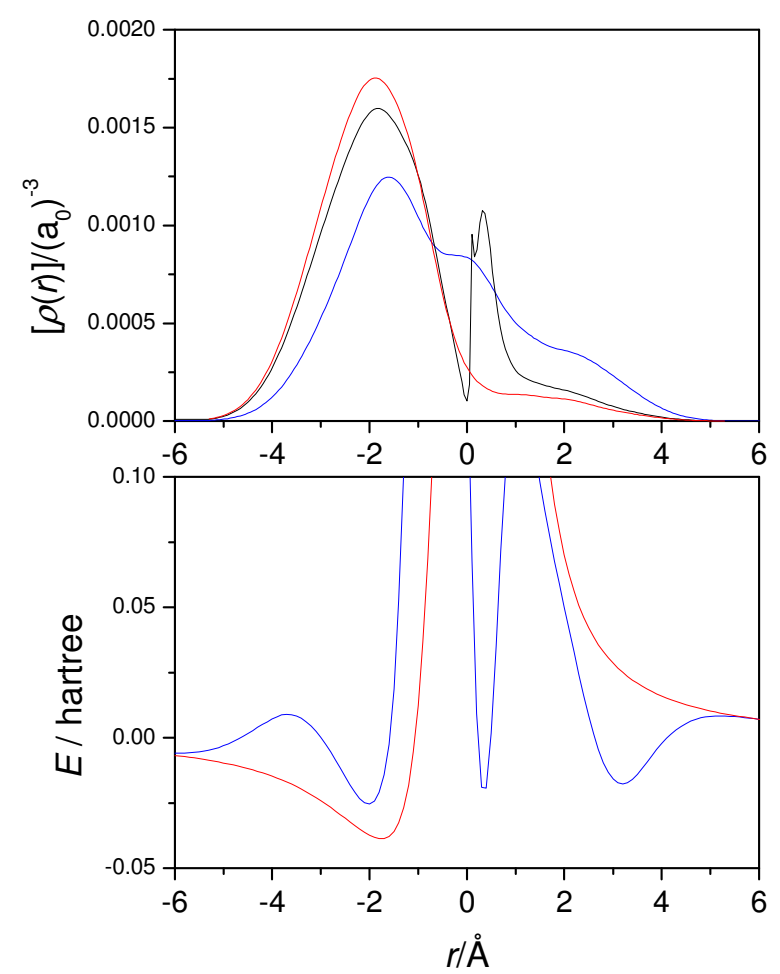


Figure 3. Turi and Rossky

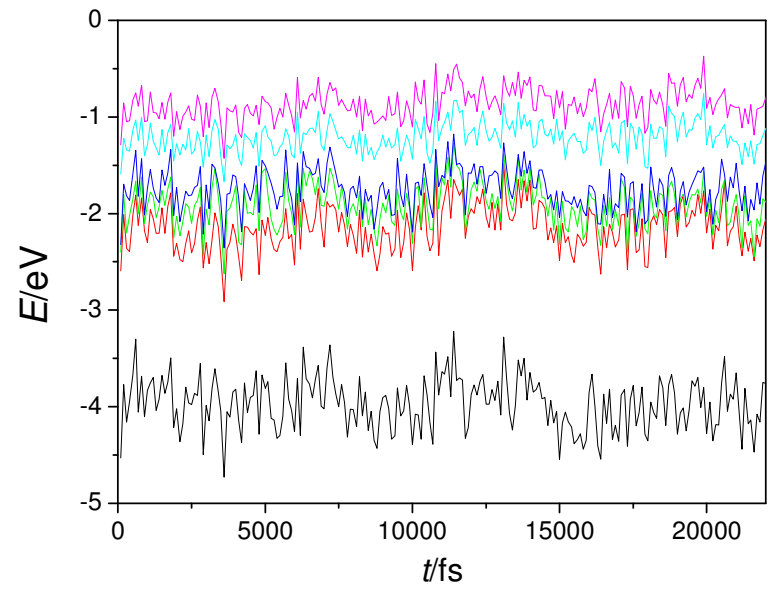


Figure 4. Turi and Rossky
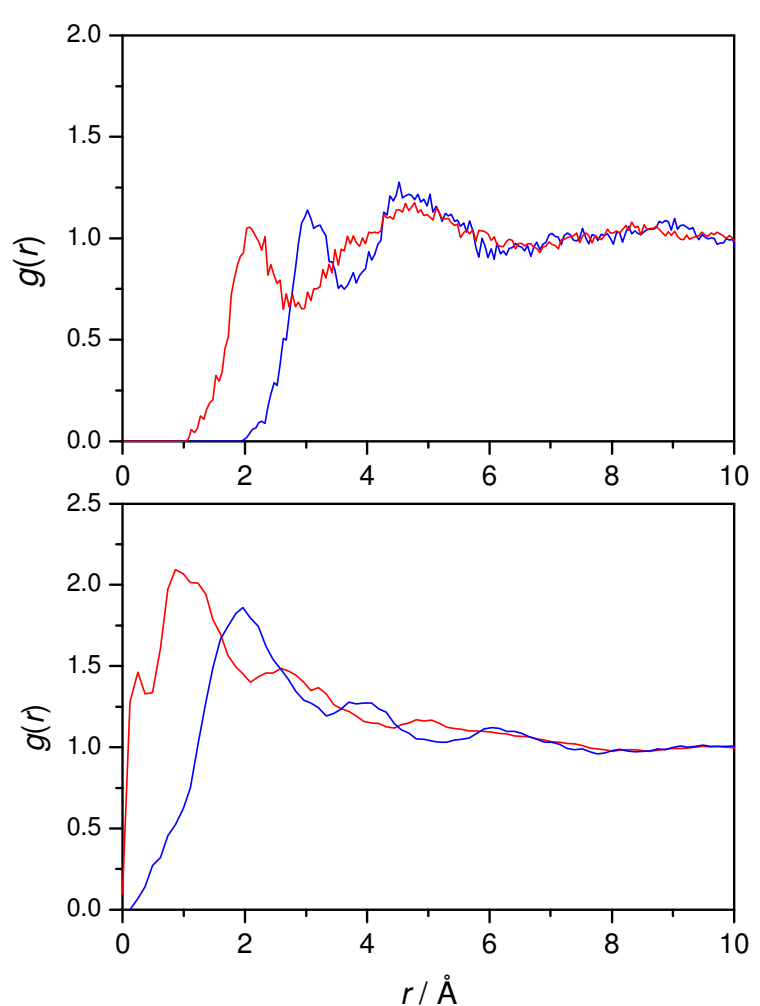
Figure 5. Turi and Rossky

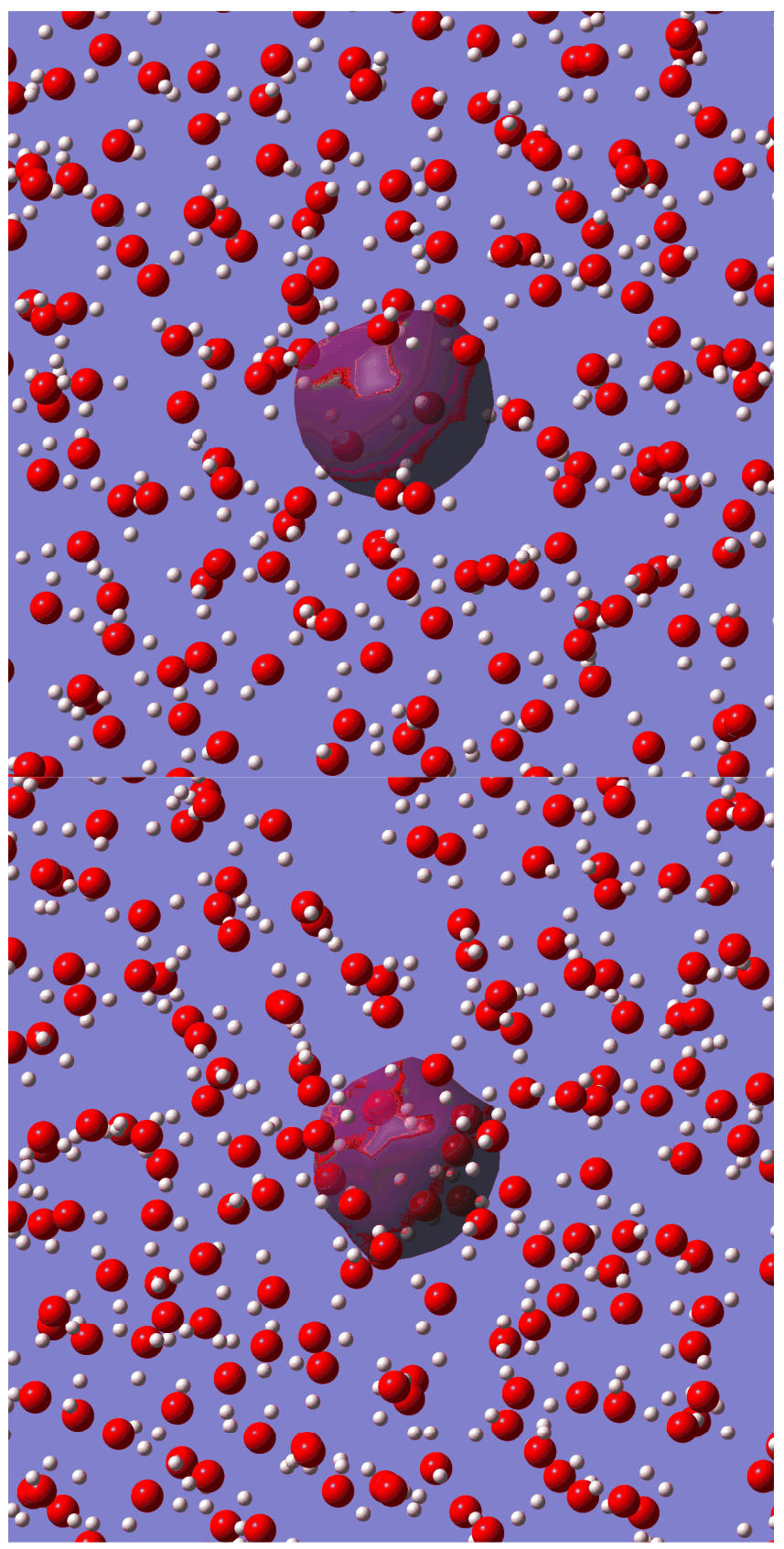


Figure 6. Turi and Rossky
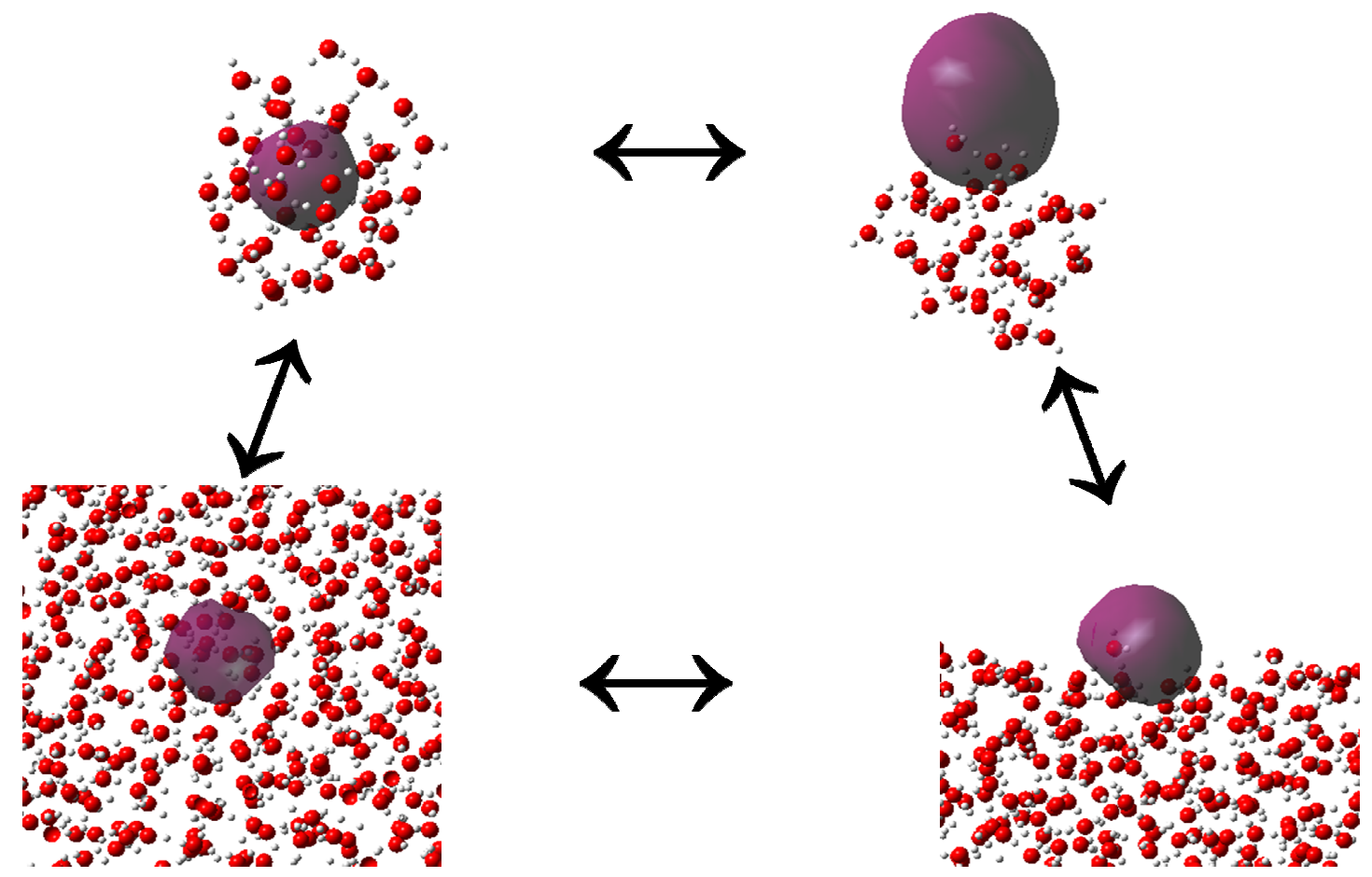
Figure 7. Turi and Rossky

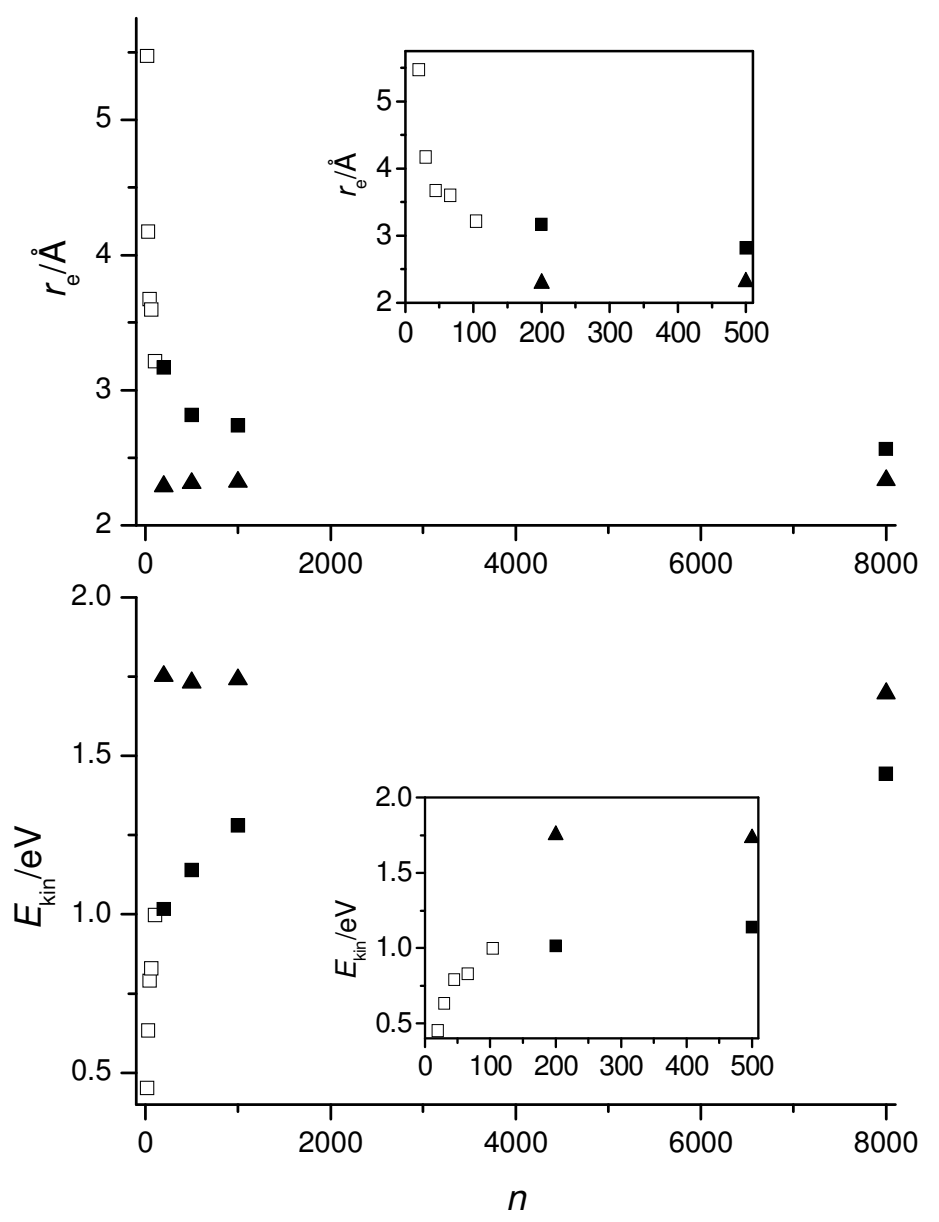


Figure 8. Turi and Rossky
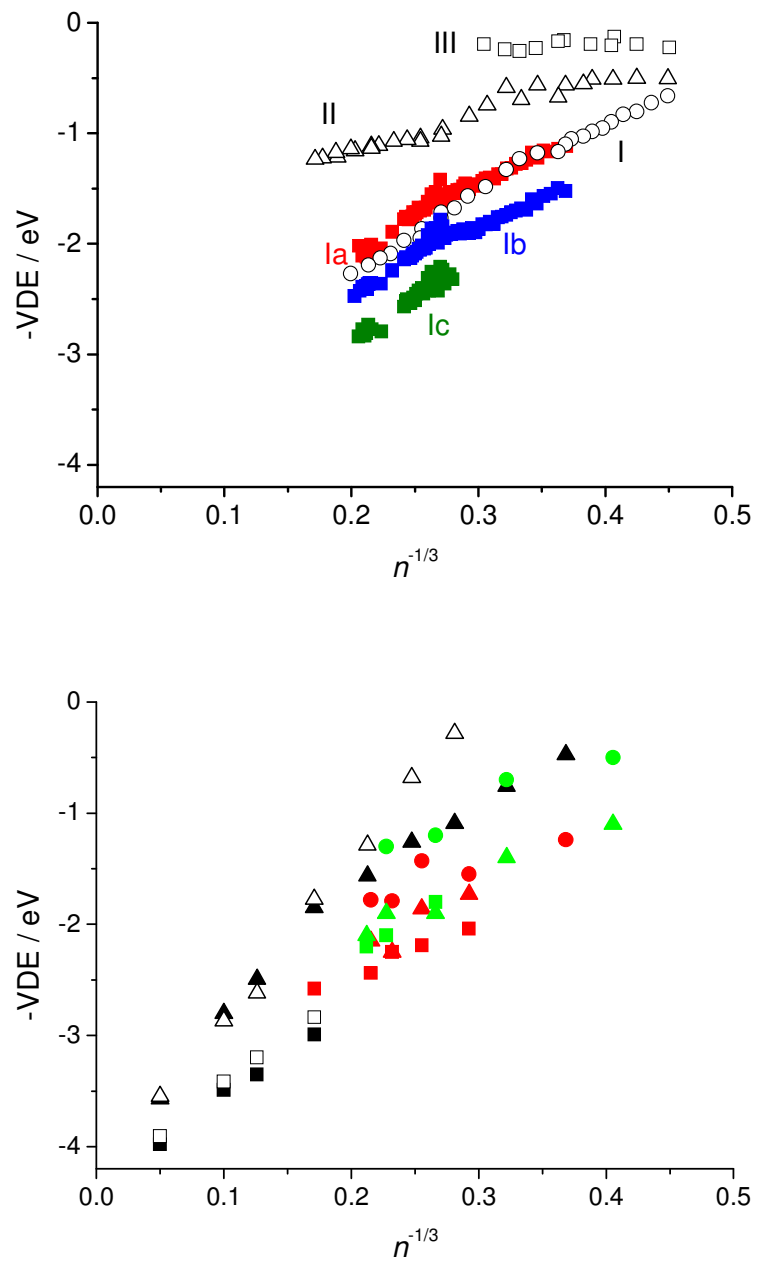
Figure 9. Turi and Rossky

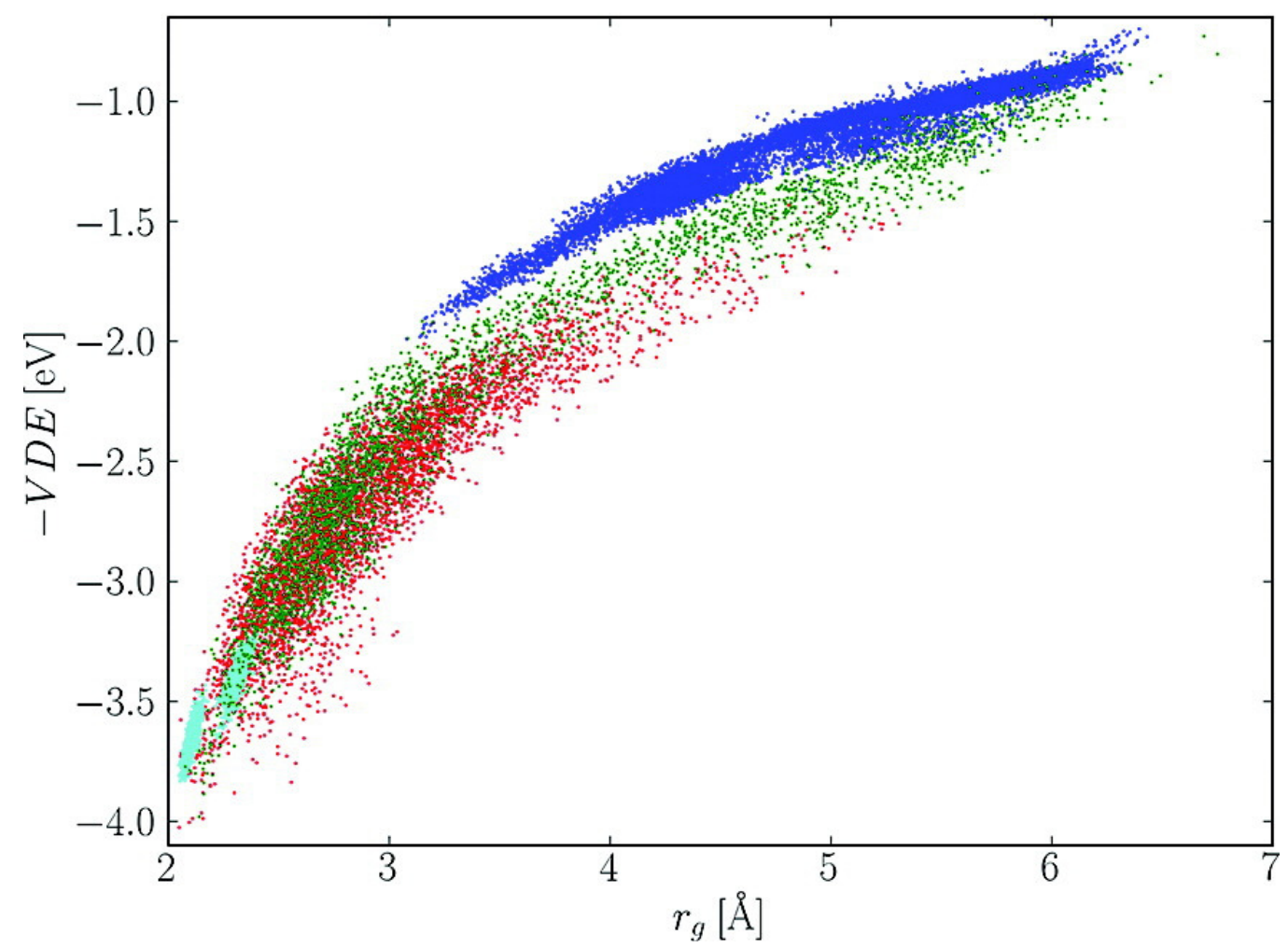


Figure 10. Turi and Rossky
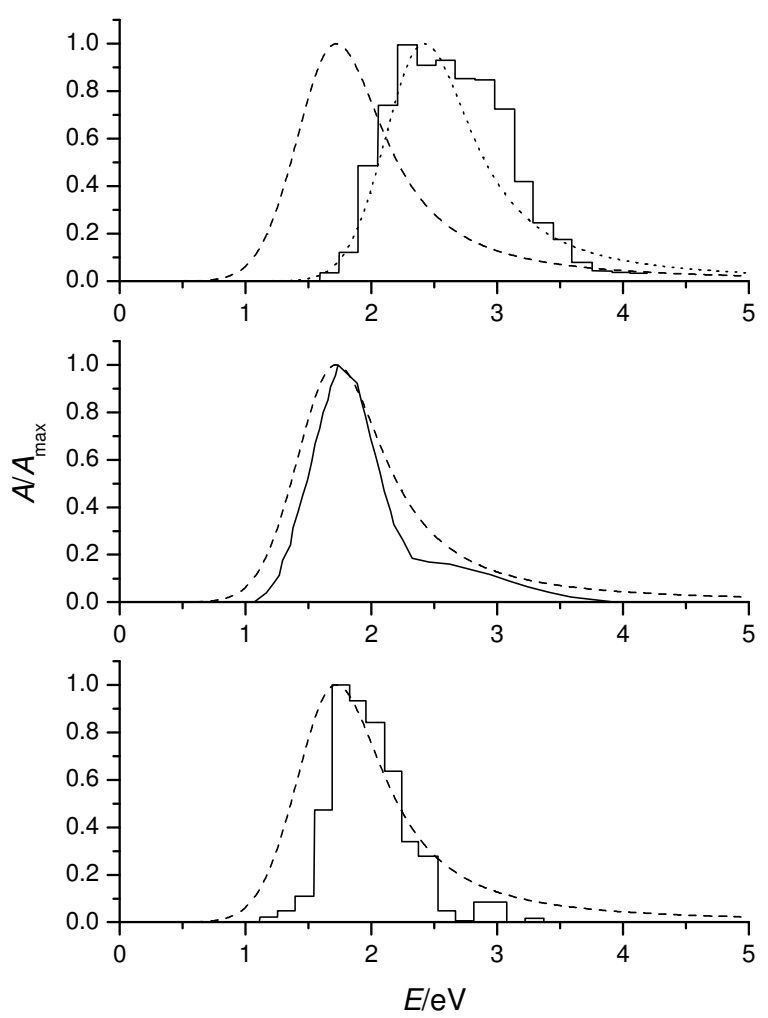
Figure 11. Turi and Rossky
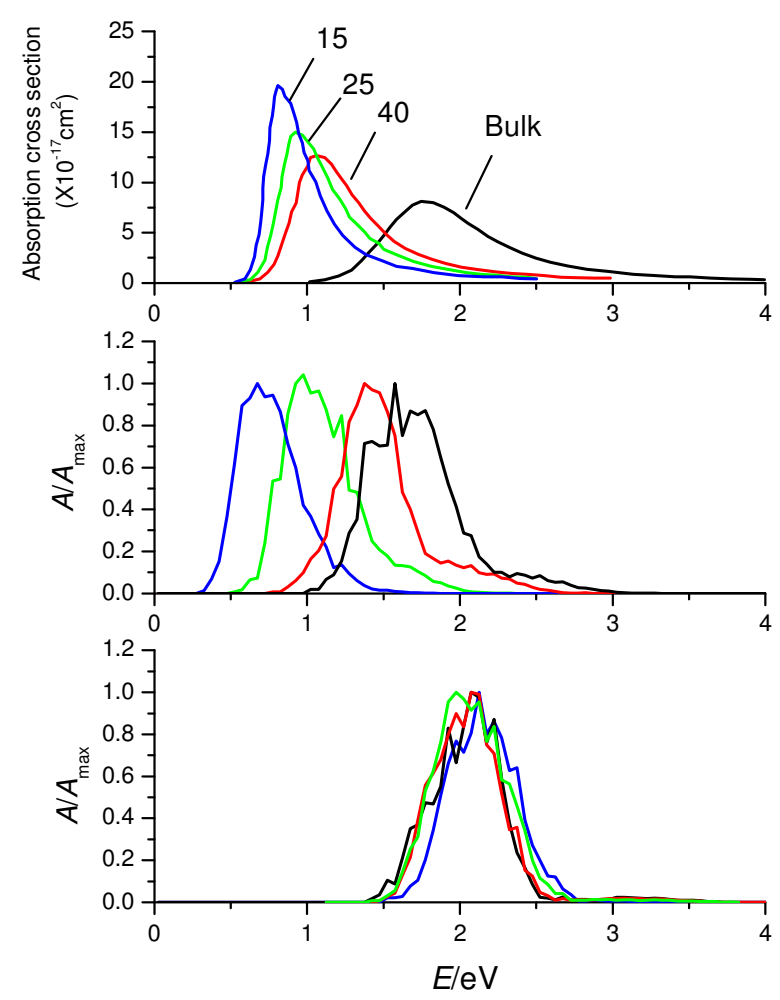
Figure 12. Turi and Rossky

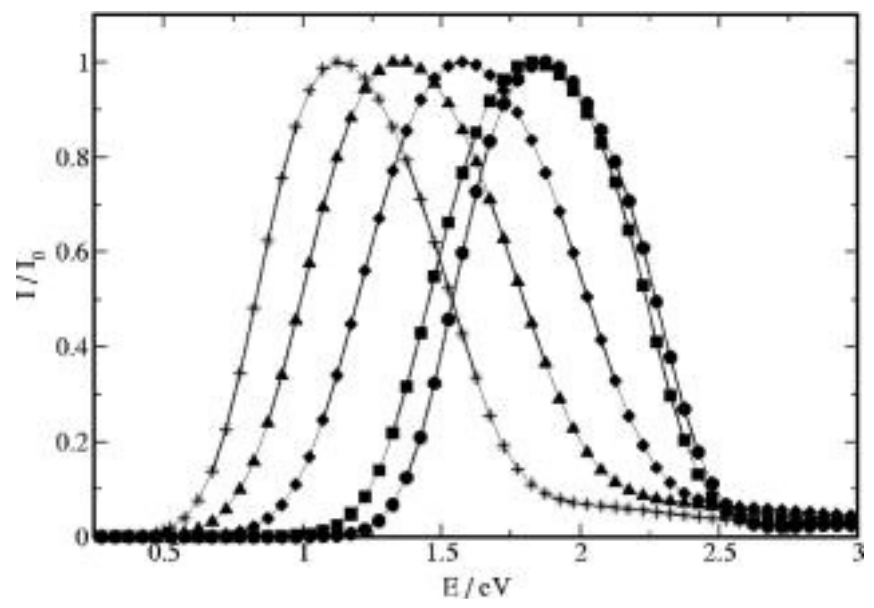


Figure 13. Turi and Rossky

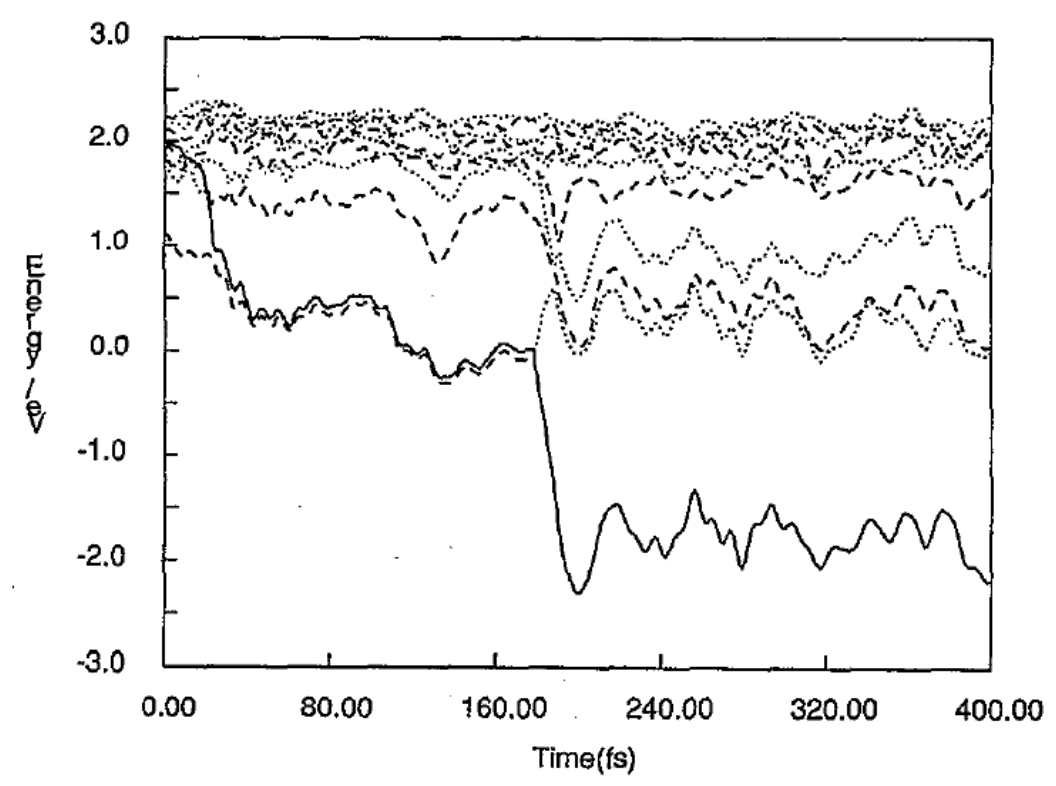


Figure 14. Turi and Rossky

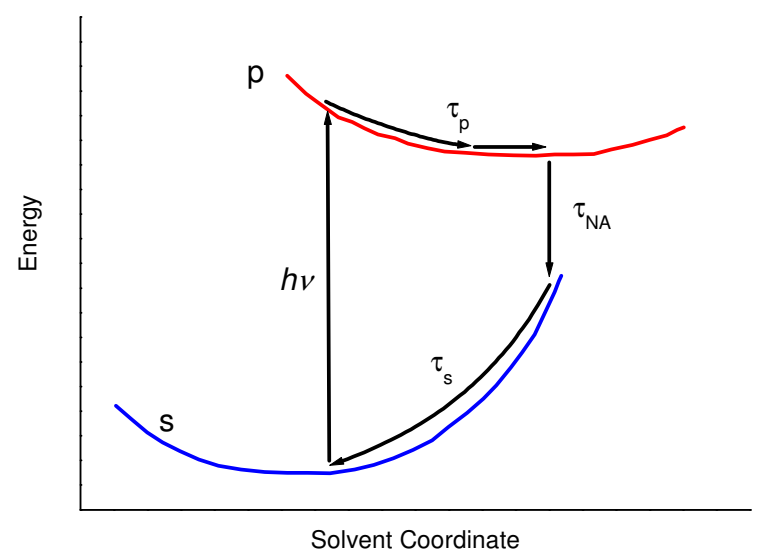


Figure 15. Turi and Rossky

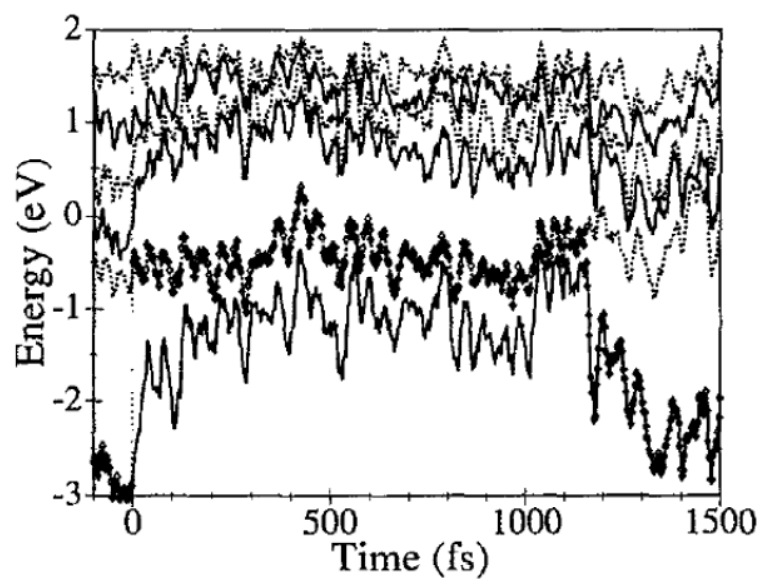


Figure 16. Turi and Rossky

a)
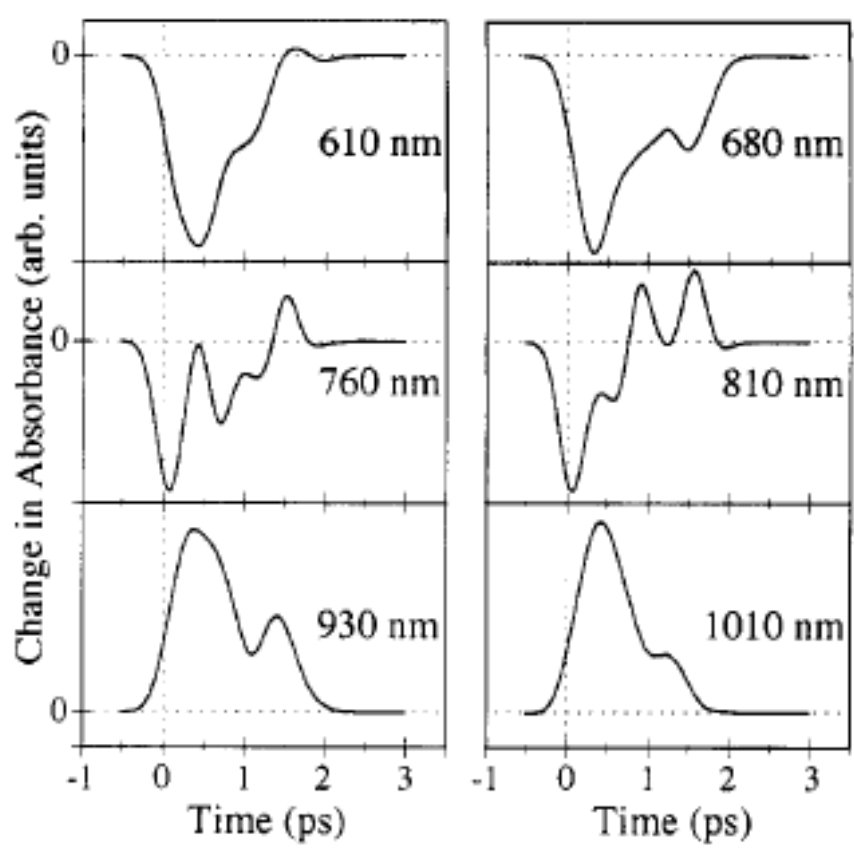

b)

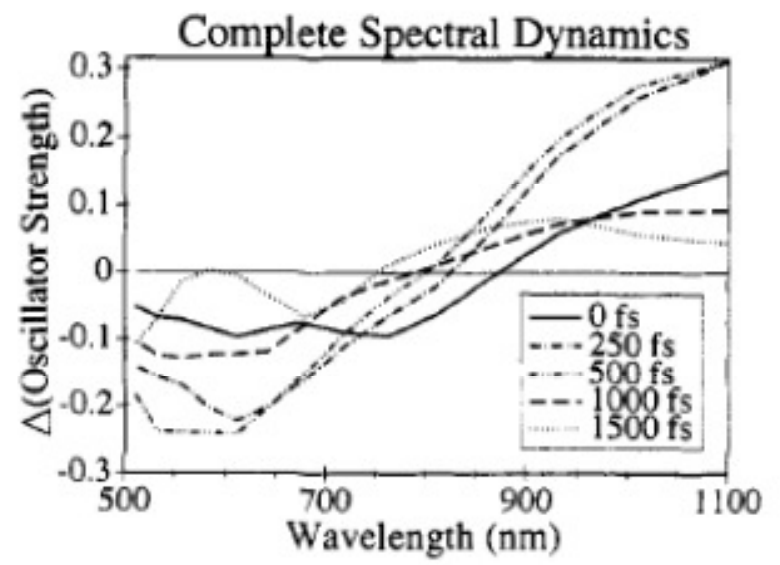


Figure 17. Turi and Rossky

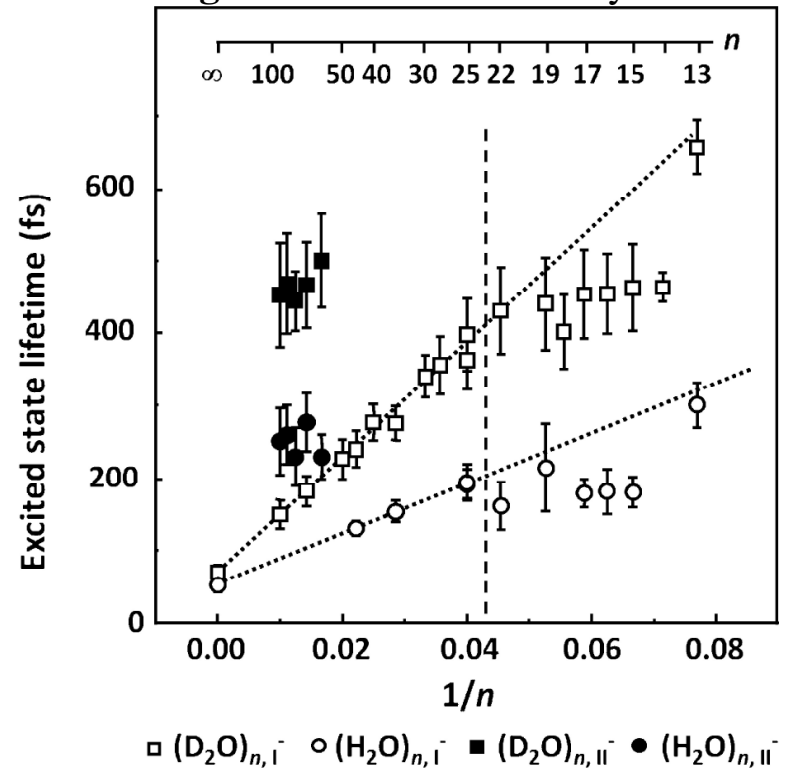


Figure 18. Turi and Rossky

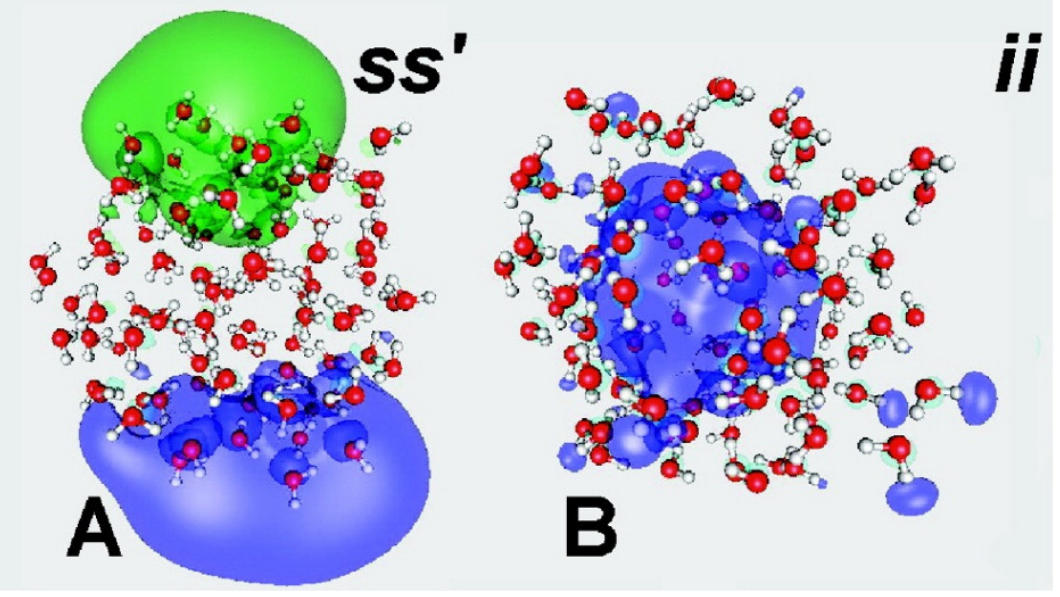




\section{References}

(1) Hart, E. J.; Boag, J. W. J. Am. Chem. Soc. 1962, 84, 4090.

(2) Weyl, W. Ann. Phys. 1864, 121, 601.

(3) Kraus, C. A. J. Am. Chem. Soc. 1908, 30, 1323.

(4) Stein, G. Discuss. Faraday Soc. 1952, 12, 227.

(5) Platzman, R. L. Basic Mechanisms in Radiobiology, (Eds. Magee, J. L.; Kamen, M. D.;

Platzman R. L.) National Research Council, Washington D.C., 1953, Publication No. 305, pp. $22-50$.

(6) Hart, E. J. Acc. Chem. Res. 1969, 2, 161.

(7) Hart, E. J.; Anbar, M. The Hydrated Electron, Wiley-Interscience: New York, NY, 1970.

(8) Garrett, B. C.; Dixon, D. A.; Camaioni, D. M.; Chipman, D. M.; Johnson, M. A.; Jonah, C. D.; Kimmel, G. A.; Miller, J. H.; Rescigno, T. N.; Rossky, P. J.; Xantheas, S. S.; Colson, S. D.; Laufer, A. H.; Ray, D.; Barbara, P. F.; Bartels, D. M.; Becker, K. H.; Bowen, H.; Bradforth, S. E.; Carmichael, I.; Coe, J. V.; Corrales, L. R.; Cowin, J. P.; Dupuis, M.; Eisenthal, K. B.; Franz, J. A.; Gutowski, M. S.; Jordan, K. D.; Kay, B. D.; LaVerne, J. A.; Lymar, S. V.; Madey, T. E.; McCurdy, C. W.; Meisel, D.; Mukamel, S.; Nilsson, A. R.;

Orlando, T. M.; Petrik, N. G.; Pimblott, S. M.; Rustad, J. R.; Schenter, G. K.; Singer, S. J.; Tokmakoff, A.; Wang, L.-S.; Wittig, C.; Zwier, T. S. Chem. Rev. 2005, 105, 355.

(9) Siefermann, K. R.; Abel, B. Angew. Chem. Int. Ed. 2011, 50, 5264.

(10) Bragg, A. E.; Verlet, J. R. R.; Kammrath, A.; Cheshnovsky, O.; Neumark, D. M. Science 2004, 306, 669.

(11) Paik, D.H.; Lee, I.-R.; Yang, D.-S.; Baskin, J. S.; Zewail, A. H. Science 2004, 306, 672.

(12) Hammer, N. I.; Shin, J-W.; Headrick, J. M.; Diken, E. G.; Roscioli, J. R.; Weddle, G. H.; Johnson, M. A. Science 2004, 306, 675. 
(13) Verlet, J. R. R.; Bragg, A. E.; Kammrath, A.; Cheshnovsky, O.; Neumark, D. M. Science 2005, 307, 93 .

(14) Turi, L.; Sheu, W.-S.; Rossky, P. J. Science 2005, 309, 914.

(15) Li, B.; Zhao, J.; Onda, K.; Jordan, K. D.; Yang, J.; Petek, H. Science 2006, 311, 1436.

(16) Wang, C.-R.; Lu, Q.-B. Angew. Chem. Intl. Ed. 2007, 46, 6316.

(17) Neumark, D. M. Nature Chem. 2010, 2, 247.

(18) Siefermann, K. R.; Liu, Y.; Lugovoy, E.; Link, O.; Faubel, M.; Buck, U.; Winter, B.;

Abel, B. Nature Chemistry, 2010, 2, 274.

(19) Larsen, R. E.; Glover, W. J.; Schwartz, B. J. Science, 2010, 329, 65.

(20) Jordan, K. D. Science 2004, 306, 618.

(21) Jordan, K. D.; Johnson, M. A. Science 2010, 329, 42.

(22) Herbert, J. M.; Jacobson, L. D. Int. Rev. Phys. Chem. 2011, 30, 1.

(23) Ben-Amotz, D. J. Phys. Chem. Lett. 2011, 2, 1216.

(24) Taub, I. A.; Eigen, K. J. Chem. Phys. 1968, 49, 2499.

(25) Kevan, L. Radiat. Phys. Chem. 1981, 17, 413. Kevan, L. J. Phys. Chem. 1981, 85, 1628.

(26) Shkrob, I. A. J. Phys. Chem. A 2007, 111, 5223.

(27) Schmidt, K. H.; Han, P.; Bartels, D. M. J. Phys. Chem. 1992, 96, 199. Schmidt, K. H.; Han, P.; Bartels, D. M. J. Phys. Chem. 1995, 99, 10530.

(28) Bronskill, M. J.; Wolff, R. K.; Hunt, J. W. J. Chem. Phys. 1970, 53, 4201. Wolff, R. K.; Bronskill, M. J.; Hunt, J. W. J. Chem. Phys. 1970, 53, 4211. Aldrich, J. E.; Bronskill, M. J.; Wolff, R. K.; Hunt, J. W. J. Chem. Phys. 1971, 55, 530.

(29) Rentzepis, P. M.; Jones, R. P.; Jortner, J. J. Chem. Phys. 1973, 59, 766.

(30) Chase, W. J.; Hunt, J. W. J. Phys. Chem. 1975, 79, 2835.

(31) Wiesenfeld, J. M.; Ippen, E. P. Chem. Phys. Lett. 1980, 73, 47.

(32) Kenney-Wallace, G. A.; Jonah, C. D. J. Phys. Chem. 1982, 86, 2572. 
(33) Migus, A.; Gauduel, Y.; Martin, J. L.; Antonetti A. Phys. Rev. Lett. 1987, 58, 1559.

(34) Long, F. H.; Lu, H.; Eisenthal, K. B. Phys. Rev. Lett. 1990, 64, 1469.

(35) Silva, C.; Walhout, P. K.; Yokoyama, K; Barbara, P. F. Phys. Rev. Lett. 1998, 80, 1086.

(36) Pshenichnikov, M. S.; Baltuska, A.; Wiersma, D. W. Chem. Phys. Lett. 2004, 389, 171.

(37) Nordlund, D.; Ogasawara, H.; Bluhm, H.; Takahashi, O.; Odelius, M.; Nagasono, M.;

Pettersson, L. G. M.; Nilsson, A. Phys. Rev. Lett. 2007, 99, 217406.

(38) Kee, T. W.; Son, D. H.; Kambhampati, P.; Barbara, P. F. J. Phys. Chem. A 2001, 105, 8434.

(39) Kambhampati, P.; Son, D. H.; Kee, T. W.; Barbara, P. F. J. Phys. Chem. A 2002, 106, 2374.

(40) Jou, F.-Y.; Freeman, G. R. J. Phys. Chem. 1977, 81, 909. Jou, F.-Y.; Freeman, G. R. Can. J. Chem. 1979, 57, 591.

(41) Jou, F.-Y.; Freeman, G. R. J. Phys. Chem. 1979, 83, 2383.

(42) Hare, P. M.; Price, E. A.; Stanisky, C. M.; Janik, I.; Bartels, D. M. J. Phys. Chem. A 2010, 114, 1766.

(43) Armbruster, M.; Haberland, H.; Schindler, H. G. Phys. Rev. Lett. 1981, 47, 323.

(44) Haberland, H.; Schindler, H. G.; Worsnop, D. R. Ber. Bunsenges. Phys. Chem. 1984, 88, 270. Haberland, H.; Langosch, H.; Schindler, H. G.; Worsnop, D. R. J. Phys. Chem. 1984, 88, 3903. Haberland, H.; Ludewigt, C.; Schindler, H. G.; Worsnop, D. R. J. Chem. Phys. 1984, 81,3742 .

(45) Knapp, M.; Echt, O.; Kreisle, D.; Recknagel, E. J. Chem. Phys. 1986, 85, 636. Knapp, M.; Echt, O.; Kreisle, D.; Recknagel, E. J. Phys. Chem. 1987, 91, 2601.

(46) Coe, J. V.; Lee, G. H.; Eaton, J. G.; Arnold, S. T.; Sarkas, H. W.; Bowen, K. H.;

Ludewigt, C.; Haberland, H.; Worsnop, D. R. J. Chem. Phys. 1990, 92, 3980.

(47) Ayotte, P.; Johnson, M. A. J. Chem. Phys. 1997, 106, 811. 
(48) Weber, J. M.; Kim, J.; Woronowicz, E. A.; Weddle, G. H.; Becker, I.; Cheshnovsky, O.; Johnson, M. A. Chem. Phys. Lett. 2001, 339, 337.

(49) Zappa, F.; Denifl, S.; Mähr, I.; Bacher, A.; Echt, O.; Märk, T. D.; Scheier, P. J. Am. Chem. Soc., 2008, 130, 5573.

(50) Gahl, C.; Bovensiepen, U.; Frischkorn, C.; Wolf, M. Phys. Rev. Lett. 2002, 89, 107402.

(51) Bovensiepen, U.; Gahl, C.; Wolf, M. J. Phys. Chem. B 2003, 107, 8706.

(52) Onda, K.; Li, B.; Zhao, J.; Jordan, K. D.; Yang, J. L.; Petek, H. Science 2005, 308, 1154.

(53) Zhao, J.; Li, B.; Onda, K.; Feng, M.; Petek, H. Chem. Rev. 2006, 106, 4402.

(54) Sagar, D. M.; Bain, C. D.; Verlet, J. R. R. J. Am. Chem. Soc. 2010, 132, 6917.

(55) Shreve, A. T.; Yen, T. A.; Neumark, D. M. Chem. Phys. Lett., 2010, 493, 216.

(56) Buchner, F.; Schultz, T.; Lübcke A. Phys. Chem. Chem. Phys. 2012, 14, 5837.

(57) Tauber, M. J.; Mathies, R. A. J. Phys. Chem. A 2001, 105, 10952. Tauber, M. J.;

Mathies, R. A. Chem. Phys. Lett. 2002, 354, 518. Tauber, M. J.; Mathies, R. A. J. Am. Chem. Soc. 2003, 125, 1394.

(58) Ogg, R. A. J. Am. Chem. Soc. 1946, 68, 155. Ogg, R. A. Phys. Rev. 1946, 69, 668.

(59) Jortner, J. J. Chem. Phys. 1959, 30, 839.

(60) Landau L. Phys. Z. Sowjetunion, 1933, 3, 664.

(61) O'Reilly, D. E. J. Chem. Phys. 1964, 41, 3736.

(62) Copeland, D. A.; Kestner, N. R.; Jortner, J. J. Chern. Phys. 1970, 53, 1189.

(63) Fueki, K.; Feng, D. F.; Kevan, L. J. Phys. Chem. 1970, 74, 1976. Fueki, K.; Feng, D. F.;

Kevan, L.; Christoffersen R. E. J. Phys. Chem. 1971, 75, 2297. Fueki, K.; Feng, D. F.;

Kevan, L. J. Am. Chem. Soc. 1973, 95, 1398.

(64) Newton, M. D. J. Chem. Phys. 1973, 58, 5833. Newton, M. D. J. Phys. Chem. 1975, 79, 2795 .

(65) Feng, D. F.; Kevan, L. Chem. Rev., 1980, 80, 1. 
(66) Makov, G.; Nitzan, A. J. Phys. Chem. 1994, 98, 3459.

(67) Zharikov, A. A.; Fischer, S. F. J. Chem. Phys. 2006, 124, 054506.

(68) Rao, B. K.; Kestner, N. R. J. Chem. Phys. 1984, 80, 1587.

(69) Clark, T.; Illing, G. J. Am. Chem. Soc. 1987, 109, 1013.

(70) Tachikawa, H.; Lund, A.; Ogasawara, M. Can. J. Chem. 1993, 71, 118.

(71) Lee, S.; Kim, J.; Lee, S. J.; Kim, K. S.; Phys. Rev. Lett. 1997, 79, 2038. Kim, K. S.; Park, I.; Lee, S.; Cho, K.; Lee, J. Y.; Kim, J.; Joannopoulos, J. D. Phys. Rev. Lett. 1996, 76, 956.

Kim, K. S.; Lee, S.; Kim, J.; Lee, J. Y.; J. Am. Chem. Soc. 1997, 119, 9329.

(72) Weigend, F.; Ahlrichs, R. Phys. Chem. Chem. Phys. 1999, 1, 4537.

(73) Herbert, J. M.; Head-Gordon, M. J. Phys. Chem. A 2005, 109, 5217.

(74) Herbert, J. M.; Head-Gordon, M. Phys. Chem. Chem. Phys. 2006, 8, 68.

(75) Williams, C. F.; Herbert, J. M. J. Phys. Chem. A 2008, 112, 6171.

(76) Khan, A. J. Chem. Phys. 2006, 125, 024307. Khan, A. Chem. Phys. Lett. 2005, 401, 85.

Khan, A. J. Chem. Phys. 2004, 121, 280.

(77) Feynman, R. P. Statistical Mechanics; Benjamin: Reading, 1972.

(78) Jonah, C. D.; Romero, C.; Rahman, A. Chem. Phys. Lett. 1986, 123, 209.

(79) Schnitker, J.; Rossky, P. J. J. Chem. Phys. 1987, 86, 3471.

(80) Barnett, R. N.; Landman, U.; Cleveland, C. L.; Jortner, J. Phys. Rev. Lett. 1987, 59, 881.

(81) Thirumalai, D.; Wallqvist, A.; Berne, B. J. J. Stat. Phys. 1986, 43, 973.

(82) Landman, U.; Barnett, R. N.; Cleveland, C. L.; Scharf, D.; Jortner, J. J. Phys. Chem. 1987, 91,4890 .

(83) Barnett, R. N.; Landman, U.; Cleveland, C. L.; Jortner, J. J. Chem. Phys. 1988, 88, 4429.

(84) Wallqvist, A.; Thirumalai, D.; Berne, B. J. J. Chem. Phys. 1986, 85, 1583.

(85) Wallqvist, A.; Thirumalai, D.; Berne, B. J. J. Chem. Phys. 1987, 86, 6404.

(86) Gallicchio, E.; Berne, B. J. J. Chem. Phys. 1996, 105, 7064. 
(87) Schnitker, J.; Motakabbir, K.; Rossky, P. J.; Friesner, R. Phys. Rev. Lett. 1988, 60, 456.

(88) Rossky, P. J.; Schnitker, J. J. Phys. Chem. 1988, 92, 4277.

(89) Barnett, R. N.; Landman, U.; Nitzan, A. J. Chem. Phys. 1988, 89, 2242.

(90) Sprik, M.; Klein, M. L. J. Chem. Phys. 1988, 89, 1592.

(91) Kotler, Z.; Neria, E.; Nitzan, A. Comput. Phys. Commun. 1991, 63, 243.

(92) Webster, F. ; Rossky, P. J.; Friesner, R. A. Comput. Phys. Commun. 1991, 63, 494.

(93) Staib, A.; Borgis, D. J. Chem. Phys. 1995, 103, 2642.

(94) Reimers, J. R.; Watts, R. O. Chem. Phys. 1984, 85, 83. Reimers, J. R.; Watts, R. O.;

Klein, M. L. Chem. Phys. 1982, 64, 95.

(95) Berendsen, H. J. C.; Postma, J. P. M.; van Gunsteren, W. F.; Hermans, J. Intermolecular Forces (Ed. Pullman, B.) Reidel, Dordrecht, 1981, pp. 331-342. Toukan, K.; Rahman, A. Phys. Rev. B 1985, 31, 2643.

(96) Jorgensen, W. L.; Chandrasekhar, J.; Madura, J. D.; Impey, R. W.; Klein, M. L. J. Chem. Phys. 1983, 79, 926.

(97) Ren, P. Y.; Ponder, J. W. J. Phys. Chem. B 2003, 107, 5933. Ren, P. Y.; Ponder, J. W. J. Phys. Chem. B 2004, 108, 13427.

(98) Del Buono, G. S.; Rossky, P. J.; Schnitker, J. J. Chem. Phys. 1991, 95, 3728.

(99) Vega, C.; Abascal, J. L. F. Phys. Chem. Chem. Phys. 2011, 13, 19663.

(100) Kiss, P. T.; Baranyai, A. J. Chem. Phys. 2009, 131, 204310.

(101) Schnitker, J.; Rossky, P. J. J. Chem. Phys. 1987, 86, 3462.

(102) Barnett, R. N.; Landman, U.; Cleveland, C. L.; Jortner, J. J. Chem. Phys. 1988, 88, 4421.

(103) Turi, L.; Borgis, D. J. Chem. Phys. 2002, 117, 6186.

(104) Jacobson, L. D.; Williams, C. F.; Herbert, J. M. J. Chem. Phys. 2009, 130, 124115.

(105) Jacobson, L. D.; Herbert, J. M. J. Chem. Phys. 2010, 133, 154506. 
(106) Morrison, M. A.; Collins, L. A. Phys. Rev. A 1978, 17, 918.

(107) Phillips, J. C.; Kleinman, L. Phys. Rev. 1959, 116, 287.

(108) Turi, L.; Gaigeot, M.-P.; Levy, N.; Borgis, D. J. Chem. Phys. 2001, 114, 7805.

(109) Larsen, R. E.; Glover, W. J.; Schwartz, B. J. J. Chem. Phys. 2009, 131, 037101.

(110) Schnitker, J.; Rossky, P. J. J. Chem. Phys. 2009, 131, 037102.

(111) Cohen, M. H.; Heine, V. Phys. Rev. 1961 122, 1821.

(112) Smallwood, C. J.; Larsen, R. E.; Glover, W. J.; Schwartz, B. J. J. Chem. Phys. 2006, $125,074102$.

(113) Turi, L.; Madarász, Á. Science, 2011, 331, 1387-c.

(114) Jacobson, L. D.; Herbert, J. M. Science, 2011, 331, 1387-d.

(115) Larsen, R. E.; Glover, W. J.; Schwartz, B. J. Science, 2011, 331, 1387-e.

(116) Herbert, J. M.; Jacobson, L. D. J. Phys. Chem. A 2011, 115, 14470.

(117) Wang, F.; Jordan, K. D. J. Chem. Phys. 2001, 114, 10717.

(118) Wang, F.; Jordan, K. D. J. Chem. Phys. 2002, 116, 6973.

(119) Wang, F.; Jordan, K. D. J. Chem. Phys. 2003, 119, 11645.

(120) Sommerfeld, T.; DeFusco, A.; Jordan, K. D. J. Phys. Chem. A 2008, 112, 11021.

(121) Xu, J.; Jordan, K. D. J. Phys. Chem. A 2010, 114, 1364.

(122) Sommerfeld, T.; Jordan, K. D. J. Phys. Chem. A 2005, 109, 11531.

(123) Sommerfeld, T.; Jordan, K. D. J. Am. Chem. Soc. 2006, 128, 5828.

(124) Sommerfeld, T.; Gardner, S. D.; DeFusco, A.; Jordan, K. D. J. Chem. Phys. 2006, 125, 174301.

(125) DeFusco, A.; Sommerfeld, T.; Jordan, K. D. Chem. Phys. Lett. 2008, 455, 135.

(126) Turi, L.; Madarász, Á.; Rossky, P. J. J. Chem. Phys. 2006, 125, 014308.

(127) Shkrob, I. A.; Glover, W. J.; Larsen, R. E.; Schwartz, B. J. J. Phys. Chem. A 2007, 111, 5232. 
(128) Jacobson, L. D.; Herbert, J. M. J. Am. Chem. Soc. 2010, 132, 10000.

(129) Park, I.; Cho, K.; Lee, S.; Kim, K. S.; Joannopoulos, J. D. Comp. Mater. Sci. 2001, 21, 291.

(130) Car, R.; Parrinello, M. Phys. Rev. Lett. 1985, 55, 2471.

(131) Boero, M. Parrinello, M.; Terakura, K.; Ikeshoji, T.; Liew, C. C. Phys. Rev. Lett. 2003, $90,226403$.

(132) Boero, M. J. Phys. Chem. A 2007, 111, 12248.

(133) Baletto, F.; Cavazzoni, C.; Scandolo, S. Phys. Rev. Lett. 2005, 95, 176801.

(134) Herbert, J. M.; Head-Gordon, M. Proc. Natl. Acad. Sci. U.S.A. 2006, 103, 14282.

(135) Frigato, T.; VandeVondele, J.; Schmidt, B.; Schütte, C.; Jungwirth, P. J. Phys. Chem. A 2008, 112, 6125 .

(136) Marsalek, O.; Frigato, T.; VandeVondele, J.; Bradforth, S. E.; Schmidt, B.; Schütte, C.; Jungwirth, P. J. Phys. Chem. B 2010, 114, 915.

(137) Marsalek, O.; Uhlig, F.; Jungwirth, P. J. Phys. Chem. C 2010, 114, 20489.

(138) Marsalek, O.; Uhlig, F.; Frigato, T.; Schmidt, B.; Jungwirth, P. Phys. Rev. Lett. 2010, $105,043002$.

(139) Marsalek, O.; Uhlig, F.; VandeVondele, J.; Jungwirth, P. Acc. Chem. Res. 2012, 45, 23.

(140) Barnett, R. N.; Giniger, R.; Cheshnovsky, O.; Landman, U. J. Phys. Chem. A. 2011, $115,7378$.

(141) Becke, A. D. Phys. Rev. A 1988, 38, 3098.

(142) Lee, C. T.; Yang, W. T.; Parr, R. G. Phys. Rev. B 1988, 37, 785.

(143) Perdew, J. P.; Burke, K.; Ernzerhof, M. Phys. Rev. Lett. 1996, 77, 3865.

(144) VandeVondele, J.; Mohamed, F.; Krack, M.; Hütter, J.; Sprik, M.; Parrinello, M. J. Chem. Phys. 2005, 122, 014515. 
(145) McGrath, M. J.; Siepmann, J. I.; Kuo, I. F. W.; Mundy, C. J. Mol. Phys. 2006, 104, 3619.

(146) VandeVondele, J.; Krack, M.; Mohamed, F.; Parrinello, M.; Chassaing, T.; Hütter, J. Comput. Phys. Commun. 2005, 167, 103.

(147) Kong, J.; Brown, S. T.; Fusti-Molnar L. J. Chem. Phys. 2006, 124, 094109.

(148) Troullier, N.; Martins, J. L. Phys. Rev. B 1991, 43, 1993.

(149) Goedecker, S.; Teter, M.; Hütter, J. Phys. Rev. B 1996, 54, 1703.

(150) Grimme, S. J. Comput. Chem. 2006, 27, 1787.

(151) Schmidt, J.; VandeVondele, J.; Kuo, I. F. W.; Sebastiani, D.; Siepmann, J. I.; Hütter, J.; Mundy, C. J. J. Phys. Chem. B 2009, 113, 11959.

(152) VandeVondele, J.; Sprik, M. Phys. Chem. Chem. Phys. 2005, 7, 1363.

(153) Larsen, R. E.; Bedard-Hearn, M. J.; Schwartz, B. J. J. Phys. Chem. B, 2006, 110, 20055.

(154) Bedard-Hearn, M. J.; Larsen, R. E.; Schwartz, B. J. J. Chem. Phys. 2005, 123, 234106.

(155) Ehrenfest, P. Z. Phys. 1927, 45, 455.

(156) Coker, D. F. Computer Simulation in Chemical Physics, (Eds. Allen, M. P.; Tildesley, D. J.) Kluwer Academic Publishers, Amsterdam, 1993, pp. 315-377.

(157) Tully, J. C.; Preston, R. K. J. Chem. Phys. 1971, 55, 562.

(158) Tully, J. C. J. Chem. Phys. 1990, 93, 1061.

(159) Pechukas, P. Phys. Rev. 1969, 181, 174.

(160) Webster, F.; Wang, E. T.; Rossky, P. J.; Friesner, R. A. J. Chem. Phys. 1994, 100, 4835. (161) Zeh, H. D. Phys. Lett. A. 1993, 172, 189. Zurek, W. H. Prog. Theor. Phys. 1993, 89, 281. Paz, J. P.; Habib, S.; Zurek, W. H. Phys. Rev. D 1993, 47, 488. Zurek, W. H.; Habib, S.; Paz, J. P. Phys. Rev. Lett. 1993, 70, 1187. Paz, J. P.; Zurek,W. H. Phys. Rev. Lett. 1999, 82, 5181. Zurek, W. H. Nature, 2001, 412, 712. Zurek, W. H. Rev. Mod. Phys. 2003, 75, 715. (162) Simonius, M. Phys. Rev. Lett. 1978, 40, 980. 
(163) Bittner, E. R.; Rossky, P. J. J. Chem. Phys. 1995, 103, 8130.

(164) Prezhdo, O. V.; Rossky, P. J. J. Chem. Phys. 1997, 107, 825.

(165) Wong, K. F.; Rossky, P. J. J. Phys. Chem. A 2001, 105, 2546.

(166) Wong, K. F.; Rossky, P. J. J. Chem. Phys. 2002, 116, 8418.

(167) Wong, K. F.; Rossky, P. J. J. Chem. Phys. 2002, 116, 8429.

(168) Hack, M. D.; Truhlar, D. G. J. Chem. Phys. 2001, 114, 9305.

(169) Zhu, C. Y.; Nangia, S.; Jasper, A. W.; Truhlar, D. G. J. Chem. Phys. 2004, 121, 7658.

(170) Jasper, A. W.; Nangia, S.; Zhu, C. Y.; Truhlar, D. G. Acc. Chem. Res. 2006, 39, 101.

(171) Fischer, S. A.; Duncan, W. R.; Prezhdo, O. V. J. Am. Chem. Soc. 2009, 131, 15483.

(172) Stier, W.; Prezhdo, O. V. J. Phys. Chem. B 2002, 106, 8047.

(173) Cohen-Tannoudji, C.; Diu, B.; Laloë, F. Quantum Mechanics, Wiley-Interscience: New York, NY, 1977.

(174) Neria, E.; Nitzan, A.; Barnett, R. N.; Landman, U. Phys. Rev. Lett. 1991, 67, 1011.

(175) Neria, E.; Nitzan, A. J. Chem. Phys. 1993, 99, 1109.

(176) Prezhdo, O. V.; Rossky, P. J. J. Chem. Phys. 1997, 107, 5863.

(177) Borgis, D.; Rossky, P. J.; Turi, L. J. Chem. Phys. 2006, 125, 064501.

(178) Borgis, D.; Lee, S. Y.; Hynes, J. T. Chem. Phys. Lett. 1989, 162, 19.

(179) Borgis, D.; Tarjus, G.; Azzouz, H. J. Phys. Chem. 1992, 96, 3188.

(180) Kubo, R. J. Math. Phys. 1963, 4, 174.

(181) Turi, L.; Hantal, G.; Rossky, P. J.; Borgis, D. J. Chem. Phys. 2009, 131, 024119.

(182) Hameka, H. F.; Robinson, G. W.; Marsden, C. J. J. Phys. Chem. 1987, 91, 3150.

(183) Tuttle, Jr., T. R.; Golden, S. J. Phys. Chem. 1991, 95, 5725.

(184) Sobolewski, A. L.; Domcke, W. Phys. Chem. Chem. Phys. 2007, 9, 3818.

(185) Sobolewski, A. L.; Domcke, W. J. Phys. Chem. 2002, 106, 4158. 
(186) Muguet, F. F. Investigations of Diffuse Intermolecular Electronic Systems, Ph.D. Thesis, Texas Tech University, 1992.

(187) Bartels, D. M. J. Chem. Phys. 2001, 115, 4404.

(188) Coe, J. V.; Williams, S. M.; Bowen, K. H. Int. Rev. Phys. Chem. 2008, 27, 27.

(189) Tang, Y.; Shen, H.; Sekiguchi, K.; Kurahashi, N.; Mizuno, T,; Suzuki, Y.-I.; Suzuki, T. Phys. Chem. Chem. Phys. 2010, 12, 3653.

(190) Madarász, Á.; Rossky, P. J.; Turi, L. J. Chem. Phys. 2009, 130, 124319.

(191) Barnett, R. N.; Landman, U.; Scharf, D.; Jortner, J. Acc. Chem. Res. 1989, 22, 350.

(192) Campagnola, P. J.; Lavrich, D. J.; DeLuca, M. J.; Johnson, M. A. J. Chem. Phys. 1991, $94,5240$.

(193) Coe, J. V.; Earhart, A. D.; Cohen, M. H.; Hoffman, G. J.; Sarkas, H. W.; Bowen, K. H. J. Chem. Phys. 1997, 107, 6023. Coe, J. V. Int. Rev. Phys. Chem. 2001, 20, 33. Coe, J. V.; Arnold, S. T.; Eaton, J. G.; Lee, G. H.; Bowen, K. H. J. Chem. Phys. 2006, 125, 014315. (194) Kim, J; Becker, I.; Cheshnovsky, O.; Johnson, M. A. Chem. Phys. Lett. 1998, 297, 90. Shin, J.-W.; Hammer, N. I.; Headrick, J. M.; Johnson, M. A. Chem. Phys. Lett. 2004, 399, 349.

(195) Griffin, G. B.; Young, R. M.; Ehrler, O. T.; Neumark, D. M. J. Chem. Phys. 2009, 131, 194302.

(196) Ma, L.; Majer, K.; Chirot, F.; von Issendorff, B. J. Chem. Phys. 2009, 131, 144303.

(197) Hammer, N. I.; Roscioli, J. R.; Johnson, M. A. J. Phys. Chem. A 2005, 109, 7896.

(198) Hammer, N. I.; Roscioli, J. R.; Bopp, J. C.; Headrick, J. M.; Johnson, M. A. J. Chem. Phys. 2005, 123, 244311.

(199) Asmis, K. R.; Santambrogio, G.; Zhou, J.; Garand, E.; Headrick, J.; Goebbert, D.; Johnson, M. A.; Neumark, D. M. J. Chem. Phys. 2007, 126, 191105. (200) Reed, A. E.; Weinstock, R. B.; Weinhold, F. J. Chem. Phys. 1985, 83, 735. 
(201) Sommerfeld, T. J. Chem. Phys. 2007, 126, 027101.

(202) Herbert, J. M.; Head-Gordon, M. J. Am. Chem. Soc. 2006, 128, 13932.

(203) Barnett, R. N.; Landman, U.; Makov, G.; Nitzan, A. J. Chem. Phys. 1990, 93, 6226.

(204) Barnett, R. N.; Landman, U.; Nitzan, A. J. Chem. Phys. 1989, 90, 4413.

(205) Madarász, Á.; Rossky, P. J.; Turi, L. J. Phys. Chem. A 2010, 114, 2331.

(206) Jacobson, L. D.; Herbert, J. M. J. Am. Chem. Soc. 2011, 133, 19889.

(207) Campagnola, P. J.; Posey, L. A.; Johnson, M. A. J. Chem. Phys. 1991, 95, 7998.

(208) Kammrath, A.; Verlet, J. R. R.; Griffin, G. B.; Neumark, D. M. J. Chem. Phys. 2006, 125,076101

(209) Neumark, D. M. Mol. Phys. 2008, 106, 2183.

(210) Bragg, A. E.; Verlet, J. R. R.; Kammrath, A.; Cheshnovsky, O.; Neumark, D. M. J. Am. Chem. Soc. 2005, 127, 15283.

(211) Ehrler, O. T.; Neumark, D. M. Acc. Chem. Res. 2009, 42, 769.

(212) Madarász, Á.; Rossky, P. J.; Turi, L. J. Chem. Phys. 2007, 126, 234707.

(213) Rodriguez, J.; Laria, D. J. Phys. Chem. B 2005, 109, 6473.

(214) Kestner, N. R. Radiation Research (Eds. Nygaard, O. F.; Adler, H. I.; Sinclair, W. K.) Academic Press, New York, 1975, pp. 333-344.

(215) Wallqvist, A.; Martyna, G.; Berne, B. J. J. Phys. Chem. 1988, 92, 1721.

(216) Berens, P. H.; White, S. R.; Wilson, K. R. J. Chem. Phys. 1981, 75, 515.

(217) Bader, J. S.; Berne, B. J. J. Chem. Phys. 1994, 100, 8359.

(218) Neumann, S.; Eisfeld, W.; Sobolewski, A. ; Domcke, W. Phys. Chem. Chem. Phys. 2004, 6, 5297.

(219) Schwartz, B. J.; Rossky, P. J. J. Chem. Phys. 1994, 101, 6902.

(220) Maroncelli, M.; Fleming, G. R. J. Chem. Phys. 1988, 89, 5044.

(221) Bernard, W.; Callen, H. B. Rev. Mod. Phys. 1959, 31, 1017. 
(222) Schwartz, B. J.; Rossky, P. J. J. Chem. Phys. 1996, 105, 6997.

(223) Mosyak, A. A.; Prezhdo, O. V.; Rossky, P. J. J. Chem. Phys. 1998, 109, 6390.

(224) Stratt, R. M. Acc. Chem. Res. 1995, 28, 201.

(225) Yang, C.-Y.; Wong, K. F.; Skaf, M. S.; Rossky, P. J. J. Chem. Phys. 2001, 114, 3598.

(226) Prezhdo, O. V.; Rossky, P. J. Phys. Rev. Lett. 1998, 81, 5294.

(227) Barnett, R. N.; Landman, U.; Nitzan, A. J. Chem. Phys. 1989, 91, 5567.

(228) Schnitker, J.; Rossky, P. J. J. Phys. Chem. 1989, 93, 6965.

(229) Barnett, R. N.; Landman, U.; Nitzan, A. J. Chem. Phys. 1990, 93, 8187.

(230) Del Buono, G. S.; Rossky, P. J.; Murphrey, T. H. J. Phys. Chem. 1992, 96, 7761.

(231) Tay, K. A.; Coudert, F.-X.; Boutin, A. J. Chem. Phys. 2008, 129, 054505.

(232) Ruff, I.; Friedrich, V. J. J. Phys. Chem. 1971, 75, 3297.

(233) Nicolas, C.; Boutin, A.; Lévy, B.; Borgis, D. J. Chem. Phys. 2003, 118, 9689.

(234) Wu, G.; Katsumura, Y.; Muroya, Y.; Li, X.; Terada, Y. Chem. Phys. Lett. 2000, 325,

531.

(235) Du, Y.; Price, E.; Bartels, D. M. Chem. Phys. Lett. 2007, 438, 234.

(236) Motakabbir, K. A.; Schnitker, J.; Rossky, P. J. J. Chem. Phys. 1989, 90, 6916.

(237) Schwartz, B. J.; Rossky, P. J. J. Phys. Chem. 1994, 98, 4489.

(238) Schwartz, B. J.; Rossky, P. J. J. Chem. Phys. 1994, 101, 6917.

(239) Schwartz, B. J.; Rossky, P. J. Phys. Rev. Lett. 1994, 72, 3282.

(240) Schwartz, B. J.; Rossky, P. J. J. Phys. Chem. 1995, 99, 2953.

(241) Bratos, S.; Leicknam, J.-C. Chem. Phys. Lett. 1996, 261, 117.

(242) Bratos, S.; Leicknam, J.-C.; Borgis, D.; Staib, A. Phys. Rev. E 1997, 55, 7217.

(243) Bratos, S.; Leicknam, J.-C. Chem. Phys. Lett. 1998, 291, 496.

(244) Bratos, S.; Leicknam, J.-C. J. Chem. Phys. 1998, 109, 9950.

(245) Shi, X. L.; Long, F. H.; Lu, H.; Eisenthal, K. B. J. Phys, Chem. 1996, 100, 11903. 
(246) Schnitker, J.; Rossky, P. J. Kenney-Wallace, G. A. J. Chem. Phys. 1986, 85, 2986.

(247) Motakabbir, K. A.; Rossky, P. J. Chem. Phys. 1989, 129, 253.

(248) Motakabbir, K. A.; Schnitker, J.; Rossky, P. J. J. Chem. Phys. 1992, 97, 2055.

(249) Barnett, R. N.; Landman, U.; Nitzan, A. J. Chem. Phys. 1990, 93, 6535.

(250) Webster, F. J.; Schnitker, J.; Friedrichs, M. S.; Friesner, R. A.; Rossky, P. J. Phys. Rev. Lett. 1991, 66, 3172.

(251) Murphrey, T. H.; Rossky, P. J. J. Chem. Phys. 1993, 99, 515.

(252) Keszei, E.; Nagy, S.; Murphrey, T. H.; Rossky, P. J. J. Chem. Phys. 1993, 99, 2004.

(253) Keszei, E.; Murphrey, T. H.; Rossky, P. J. J. Phys. Chem. 1995, 99, 22.

(254) Alfano, J. C.; Walhout, P. K.; Kimura, Y.; Barbara, P. F. J. Chem. Phys. 1993, 98, 5996.

(255) Kimura, Y.; Alfano, J. C.; Walhout, P. K.; Barbara, P. F. J. Phys. Chem. 1994, 98, 3450.

(256) Yokoyama, K.; Silva, C.; Son, D. H.; Walhout, P. K.; Barbara, P. F. J. Phys. Chem. A 1998, 102, 6957.

(257) Reid, P. J.; Silva, C.; Walhout, P. K.; Barbara, P. F. Chem. Phys. Lett. 1994, 228, 658.

(258) Assel, M.; Laenen, R.; Laubereau, A. J. Phys. Chem. A 1998, 102, 2256.

(259) Assel, M.; Laenen, R.; Laubereau, A. J. Chem. Phys. 1999, 111, 6869.

(260) Assel, M.; Laenen, R.; Laubereau, A. Chem. Phys. Lett. 2000, 317, 13.

(261) Thaller, A.; Laenen, R.; Laubereau, A. Chem. Phys. Lett. 2004, 398, 459.

(262) Wang, C.-R.; Hu, A.; Lu, Q.-B. J. Chem. Phys. 2006, 124, 241102.

(263) Borgis, D.; Rossky, P. J.; Turi, L. J. Chem. Phys. 2007, 127, 174508.

(264) Prezhdo, O. V.; Rossky, P. J. J. Phys. Chem. 1996, 100, 17094.

(265) Schwartz, B. J.; Bittner, E. R.; Prezhdo, O. V.; Rossky, P. J. J. Chem. Phys. 1996, 104, 5942. 
(266) Shkrob, I. Chem. Phys. Lett. 2008, 467, 84.

(267) Cavanagh, M. C.; Martini, I. B.; Schwartz, B. J. Chem. Phys. Lett. 2004, 396, 359.

(268) Fischer, S. F.; Dietz, W. Z. Phys. Chem. 2007, 221, 585.

(269) Stähler, J.; Bovensiepen, U.; Meyer, M.; Wolf, M. Chem. Soc. Rev. 2008, 37, 2180.

(270) Stähler, J.; Gahl, C.; Bovensiepen, U.; Wolf, M. J. Phys. Chem. B 2006, 110, 9637.

(271) Bovensiepen, U.; Gahl, C.; Stähler, J.; Bockstedte, M.; Meyer, M.; Baletto, F.;

Scandolo, S.; Zhu, X.-Y.; Rubio, A.; Wolf, M. J. Phys. Chem. C 2009, 113, 979.

(272) Stähler, J.; Meyer, M.; Bovensiepen, U.; Wolf, M. Chem. Sci. 2011, 2, 907.

(273) Jortner, J.; Levine, R.; Ottolenghi, M.; Stein, G. J. Phys. Chem. 1961, 65, 1232.

(274) Jortner, J.; Ottolenghi, M.; Stein, G. J. Phys. Chem. 1964, 68, 247.

(275) Long, F. H. Shi, X. L.; Lu, H.; Eisenthal, K. B. J. Phys, Chem. 1994, 98, 7252.

(276) Gauduel, Y.; Gelabert, H.; Ashokkumar, M. Chem. Phys. 1995, 197, 167.

(277) Kloepfer, J. A.; Vilchiz, V. H.; Lenchenkov, V. A.; Bradforth, S. E. Chem. Phys. Lett.

1998, 298, 120. Kloepfer, J. A.; Vilchiz, V. H.; Lenchenkov, V. A.; Germaine, A. C.;

Bradforth, S. E. J. Chem. Phys. 2000, 113, 6288.

(278) Iglev, H.; Trifonov, A.; Thaller, A.; Buchvarov, L.; Fiebig, T.; Laubereau, A. Chem. Phys. Lett. 2005, 403, 198.

(279) Moskun, A. C.; Bradforth, S. E.; Thogersen, J.; Keiding, S. J. Phys. Chem. A 2006, 110, 10947.

(280) Serxner, D.; Dessent, C. E. H.; Johnson, M. A. J. Chem. Phys. 1996, 105, 7231.

(281) Lehr, L.; Zanni, M. T.; Frischkorn, C.; Weinkauf, R.; Neumark, D. M. Science 1999, $284,635$.

(282) Verlet, J. R. R.; Kammarth, A.; Griffin, G. B.; Neumark, D. M. J. Chem. Phys. 2005, $123,231102$. 
(283) Pham, V.-T.; Penfold, T. J.; van der Veen, R. M.; Lima, F.; El Nahhas, A.; Johnson, S. L.; Beaud, P.; Abela, R.; Bressler, C.; Tavernelli, I.; Milne, C. J.; Chergui, M. J. Am. Chem. Soc. 2011, 133, 12740.

(284) Sheu, W.-S.; Rossky, P. J. J. Am. Chem. Soc. 1993, 115, 7729.

(285) Sheu, W.-S.; Rossky, P. J. Chem. Phys. Lett. 1993, 202, 186.

(286) Sheu, W.-S.; Rossky, P. J. Chem. Phys. Lett. 1993, 213, 233.

(287) Sheu, W.-S.; Rossky, P. J. J. Phys. Chem. 1996, 100, 1295.

(288) Staib, A.; Borgis, D. J. Chem. Phys. 1996, 104, 9027.

(289) Pham, V.-T.; Tavernelli, I.; Milne, C. J.; van der Veen, R. M.; D’Angelo, P.; Bressler, C.; Chergui, M. Chem. Phys. 2010, 371, 24.

(290) Marx, D.; Tuckermann, M. E.; Hutter, J. ; Parrinello, M. Nature, 1999, 397, 601.

(291) Han, P.; Bartels, D. M. J. Phys. Chem. 1992, 96, 4899.

(292) Shiraishi, H.; Sunaryo, G. R.; Ishigure, K. J. Phys. Chem. 1994, 98, 5164.

(293) Renault, J. P.; Vuilleumier, R.; Pommeret, S. J. Phys. Chem. A 2008, 112, 7027.

(294) Zimbrick, J.; Kevan, L. J. Am. Chem. Soc. 1967, 89, 2483.

(295) Fueki, K. J. Chem. Phys. 1969, 50, 5381.

(296) Basco, N.; Kenney-Wallace, G. A.; Vidyarthi, S. K.; Walker, D. C. Can. J. Chem. 1972, $50,2059$.

(297) Meisel, D.; Czapski, G.; Matheson, M. S.; Mullac, W. A. Int. J. Rad. Phys. Chem. 1975, 7, 233.

(298) Ferradini, C.; Jay-Gerin, J.-P. Radiat. Phys. Chem. 1993, 41, 487.

(299) Feng, D.-F.; Fueki, K.; Kevan, L. J. Chem. Phys. 1973, 58, 3281.

(300) Kaukonen, H.-P.; Barnett, R. N.; Landman, U. J. Chem. Phys. 1992, 97, 1365.

(301) Rajagopal, G.; Barnett, R. N.; Landman, U. Phys. Rev. Lett. 1991, 67, 727.

(302) Larsen, R. E.; Schwartz, B. J. J. Phys. Chem. B 2004, 108, 11760. 
(303) Larsen, R. E.; Schwartz, B. J. J. Phys. Chem. B 2006, 110, 1006.

(304) Larsen, R. E.; Schwartz, B. J. J. Phys. Chem. B 2006, 110, 9681.

(305) Larsen, R. E.; Schwartz, B. J. J. Phys. Chem. B 2006, 110, 9692. 